\title{
Correlated-Electron Systems and High-Temperature Superconductivity
}

\author{
Takashi Yanagisawa $^{1}$, Mitake Miyazaki ${ }^{2}$, Kunihiko Yamaji ${ }^{1}$ \\ ${ }^{1}$ Electronics and Photonics Research Group, National Institute of Advanced Industrial Science and Technology (AIST), \\ Tsukuba Central 2, Tsukuba, Japan \\ ${ }^{2}$ Hakodate National College of Technology, Hakodate, Japan \\ Email: t-yanagisawa@aist.go.jp
}

Received April 2, 2013; revised May 5, 2013; accepted June 1, 2013

Copyright (C) 2013 Takashi Yanagisawa et al. This is an open access article distributed under the Creative Commons Attribution License, which permits unrestricted use, distribution, and reproduction in any medium, provided the original work is properly cited.

\begin{abstract}
We present recent theoretical results on superconductivity in correlated-electron systems, especially in the twodimensional Hubbard model and the three-band d-p model. The mechanism of superconductivity in high-temperature superconductors has been extensively studied on the basis of various electronic models and also electron-phonon models. In this study, we investigate the properties of superconductivity in correlated-electron systems by using numerical methods such as the variational Monte Carlo method and the quantum Monte Carlo method. The Hubbard model is one of basic models for strongly correlated electron systems, and is regarded as the model of cuprate high temperature superconductors. The d-p model is more realistic model for cuprates. The superconducting condensation energy obtained by adopting the Gutzwiller ansatz is in reasonable agreement with the condensation energy estimated for $\mathrm{YBa}_{2} \mathrm{Cu}_{3} \mathrm{O}_{7}$. We show the phase diagram of the ground state using this method. We have further investigated the stability of striped and checkerboard states in the under-doped region. Holes doped in a half-filled square lattice lead to an incommensurate spin and charge density wave. The relationship of the hole density $x$ and incommensurability $\delta$, $\delta \sim x$, is satisfied in the lower doping region, as indicated by the variational Monte Carlo calculations for the two-dimensional Hubbard model. A checkerboard-like charge-density modulation with a roughly $4 \times 4$ period has also been observed by scanning tunneling microscopy experiments in Bi2212 and Na-CCOC compounds. We have performed a variational Monte Carlo simulation on a two-dimensional $t-t^{\prime}-t^{\prime \prime}-U$ Hubbard model with a Bi-2212 type band structure and found that the $4 \times 4$ period checkerboard spin modulation, that is characterized by multi Q vectors, is indeed stabilized. We have further performed an investigation by using a quantum Monte Carlo method, which is a numerical method that can be used to simulate the behavior of correlated electron systems. We present a new algorithm of the quantum Monte Carlo diagonalization that is a method for the evaluation of expectation value without the negative sign problem. We compute pair correlation functions and show that pair correlation is indeed enhanced with hole doping.
\end{abstract}

Keywords: High-Temperature Superconductivity; Strongly Correlated Electrons; Monte Carlo Methods; Hubbard Model; Condensation Energy; Pair-Correlation Function

\section{Introduction}

The effect of the strong correlation between electrons is important for many quantum critical phenomena, such as unconventional superconductivity (SC) and the metalinsulator transition. Typical correlated electron systems are high-temperature superconductors [1-5], heavy fermions [6-9] and organic conductors [10]. The phase diagram for the typical high- $T_{c}$ cuprates is shown in Figure 1 [9]. It has a characteristics that the region of antiferromagnetic order exists at low carrier concentra- tions and the superconducting phase is adjacent to the antiferromagnetism.

In the low-carrier region shown in Figure 2, there is the anomalous metallic region where the susceptibility and $1 / T_{1}$ show a peak above $T_{c}$ suggesting an existence of the pseudogap. To clarify an origin of the anomalous metallic behaviors is also a subject attracting many physicists as a challenging problem.

It has been established that the Cooper pairs of high- $T_{c}$ cuprates have the $d$-wave symmetry in the hole-doped materials $[11,12]$. Several evidences of $d$-wave pairing 


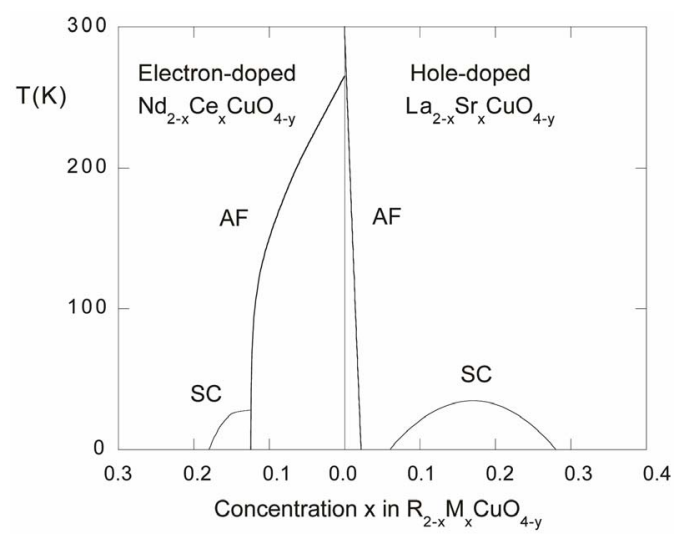

Figure 1. Phase diagram delineating the regions of superconductivity and antiferromagnetic ordering of the $\mathrm{Cu}^{2+}$ ions for the hole-doped $\mathrm{La}_{2-x} \mathrm{Sr}_{x} \mathrm{CuO}_{4}$ and electron-doped $\mathrm{Nd}_{2-x} \mathrm{Ce}_{x} \mathrm{CuO}_{4-y}$ systems.

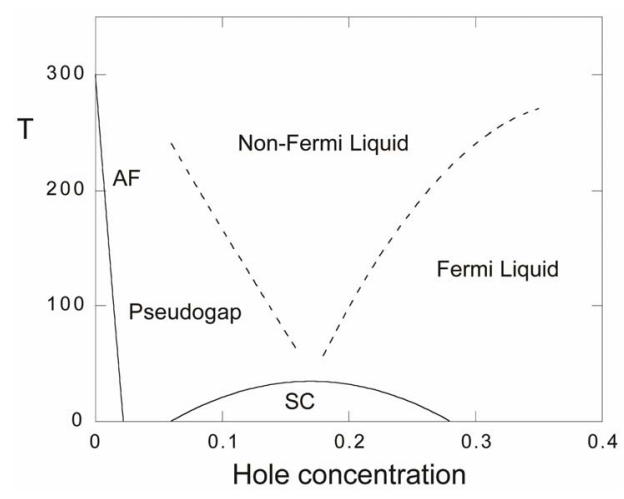

Figure 2. Phase diagram showing the regions of non-Fermi liquid and pseudogap metal for the hole-doped case. The boundaries indicated in the figure are not confirmed yet.

symmetry were provided for the electron-doped cuprates $\mathrm{Nd}_{2-x} \mathrm{Ce}_{x} \mathrm{CuO}_{4}$ [13-15]. Thus it is expected that the superconductivity of electronic origin is a candidate for the high- $T_{c}$ superconductivity. We can also expect that the origin of $d$-wave superconductivity lies in the onsite Coulomb interaction of the two-dimensional Hubbard model.

The antiferromagnetism should also be examined in correlated electron systems. The undoped oxide compounds exhibit rich structures of antiferromagnetic correlations over a wide range of temperature that are described by the two-dimensional quantum antiferromagnetism [16-18]. A small number of holes introduced by doping are responsible for the disappearance of long-range antiferromagnetic order [19-24].

Recent neutron scattering experiments have suggested an existence of incommensurate ground states with modulation vectors given by $Q_{s}=(\pi \pm 2 \pi \delta, \pi)$ and $Q_{c}=( \pm 4 \pi \delta, 0)$ (or $Q_{s}=(\pi, \pi \pm 2 \pi \delta)$ and $\left.Q_{c}=(0, \pm 4 \pi \delta)\right)$ where $\delta$ denotes the hole-doping ratio [25]. We can expect that the incommensurate cor- relations are induced by holes doped into the $\mathrm{Cu}-\mathrm{O}$ plane in the underdoped region. A checkerboard-like charge-density modulation with a roughly $4 \times 4$ period has also been observed by scanning tunneling microscopy experiments in Bi2212 and Na-CCOC compounds.

Recently the mechanism of superconductivity in hightemperature superconductors has been extensively studied using various two-dimensional (2D) models of electronic interactions. Among them the 2D Hubbard model [26] is the simplest and most fundamental model. This model has been studied intensively using numerical tools, such as the Quantum Monte Carlo method [27-42], and the variational Monte Carlo method [24,43-50]. The two-leg ladder Hubbard model was also investigated with respect to the mechanism of high-temperature superconductivity [51-59].

Since the discovery of cuprate high-temperature superconductors, many researchers have tried to explain the occurrence of superconductivity of these materials in terms of the two-dimensional (2D) Hubbard model. However, it remains matter of considerable controversial as to whether the 2D Hubbard model accounts for the properties of high-temperature cuprate superconductors. This is because the membership of the the two-dimensional Hubbard model in the category of strongly correlated systems is a considerable barrier to progress on this problem. The quest for the existence of a superconducting transition in the 2D Hubbard model is a long-standing problem in correlated-electron physics, and has been the subject of intensive study $[35,36,38,46$, $60,61]$. In particular, the results of quantum Monte Carlo methods, which are believed to be exact unbiased methods, have failed to show the existence of superconductivity in this model $[38,61]$.

In the weak coupling limit we can answer this question. We can obtain the superconducting order parameter of the Hubbard model in the limit of small $U$, that is given by [62-66]

$$
\Delta=\exp \left(-\frac{2}{x U^{2}}\right),
$$

where $U$ is the strength of the on-site Coulomb interaction and the exponent $x$ is determined by solving the gap equation. Thus the existence of the superconducting gap is guaranteed by the weak coupling theory although $\Delta$ is extremely small because of the exponential behavior given above. $x$ indicates the strength of superconductivity. In the intermediate or large coupling region, we must study it beyond the perturbation theory.

We investigate the ground state of the Hubbard model by employing the variational Monte Carlo method. In the region $6 \leq U \leq 12$, the finite superconducting gap is 
obtained by using the quantum variational Monte Carlo method. The superconducting condensation energy obtained by adopting the Gutzwiller ansatz is in reasonable agreement with the condensation energy derived for $\mathrm{YBa}_{2} \mathrm{Cu}_{3} \mathrm{O}_{7}$. We have further investigated the stability of striped and checkerboard states in the under-doped region. Holes doped in a half-filled square lattice lead to an incommensurate spin and charge density wave. The relationship of the hole density $\mathrm{x}$ and incommensurability $\delta, \delta \sim x$, is satisfied in the lower doping region. This is consistent with the results by neutron scattering measurements. To examine the stability of a $4 \times 4$ checkerboard state, we have performed a variational Monte Carlo simulation on a two-dimensional $t-t^{\prime}-t^{\prime \prime}-U$ Hubbard model with a Bi-2212 type band structure. We found that the $4 \times 4$ period checkerboard checkerboard spin modulation that is characterized by multi $Q$ vectors is stabilized.

Further investigation has been performed by using the quantum Monte Carlo method which is a numerical method that can be used to simulate the behavior of correlated electron systems. This method is believed to be an exact unbiased method. We compute pair correlation functions to examine a possibility of superconductivity.

The Quantum Monte Carlo (QMC) method is a numerical method employed to simulate the behavior of correlated electron systems. It is well known, however, that there are significant issues associated with the application to the QMC. First, the standard Metropolis (or heat bath) algorithm is associated with the negative sign problem. Second, the convergence of the trial wave function is sometimes not monotonic, and further, is sometimes slow. In past studies, workers have investigated the possibility of eliminating the negative sign problem [37,38,40-42]. We present the results obtained by a method, quantum Monte Carlo diagonalization, without the negative sign problem.

\section{Hubbard Hamiltonian}

The Hubbard Hamiltonian is

$$
H=-\sum_{i j} t_{i j} c_{i \sigma}^{\dagger} c_{i \sigma}+U \sum_{i} n_{i \uparrow} n_{i \downarrow}
$$

where $c_{i \sigma}^{\dagger}$ and $c_{i \sigma}$ denote the creation and annihilation operators of electrons, respectively, and $n_{i \sigma}=c_{i \sigma}^{\dagger} c_{i \sigma}$ is the number operator. The second term represents the on-site Coulomb interaction which acts when the two electrons occupy the same site. The numbers of lattice sites and electrons are denoted as $N$ and $N_{e}$, respectively. The electron density is $n_{e}=N_{e} / N$.

In the non-interacting limit $U=0$, the Hamiltonian is easily diagonalized in terms of the Fourier transformation. In the ground state each energy level is occupied by electrons up to the Fermi energy. In the other limit $t_{i j}=0$, each site is occupied by the up- or down-spin electron, or is empty. The non-zero $t_{i j}$ induces the movement of electrons that leads to a metallic state id $N_{e} \neq N$. The ground state is probably insulating at half-filling $N_{e}=N$ if $U$ is sufficiently large.

If $t_{i j}=t$ are non-zero only for the nearest-neighbor pairs, the Hubbard Hamiltonian is transformed to the following effective Hamiltonian for large $U / t$ [67]:

$$
\begin{aligned}
H= & -t \sum_{\langle i j\rangle \sigma} a_{i \sigma}^{\dagger} a_{j \sigma}-\frac{t^{2}}{U} \sum_{\mu \mu^{\prime}}\left[a_{j+\mu \uparrow}^{\dagger} a_{\downarrow}^{\dagger} a_{j \downarrow} a_{j+\mu^{\prime} \uparrow}\right. \\
& +a_{j \uparrow}^{\dagger} a_{j+\mu \downarrow}^{\dagger} a_{j+\mu^{\prime} \downarrow} a_{j \uparrow}+a_{j+\mu \uparrow}^{\dagger} a_{j \downarrow}^{\dagger} a_{j+\mu^{\prime} \downarrow} a_{j \uparrow} \\
& \left.+a_{j \uparrow}^{\dagger} a_{j+\mu \downarrow}^{\dagger} a_{j \downarrow} a_{j+\mu \uparrow}\right],
\end{aligned}
$$

where $a_{i \sigma}=c_{i \sigma}\left(1-n_{i,-\sigma}\right)$ and $j+\mu$ and $j+\mu^{\prime}$ indicate the nearest-neighbor sites in the $\mu$ and $\mu^{\prime}$ directions, respectively. The second term contains the three-site terms when $\mu \neq \mu^{\prime}$. If we neglect the three-site terms, this effective Hamiltonian is reduced to the $t-J$ model:

$$
H=-t \sum_{\langle i j\rangle \sigma}\left(a_{i \sigma}^{\dagger} a_{j \sigma}+\text { h.c. }\right)+J \sum_{\langle i j\rangle}\left(\boldsymbol{S}_{i} \cdot \boldsymbol{S}_{j}-\frac{1}{4} n_{i} n_{j}\right),
$$

where $J=4 t^{2} / U$.

The Hubbard model has a long history in describing the magnetism of materials since the early works by Hubbard [26], Gutzwiller [68] and Kanamori [69]. Onedimensional Hubbard model has been well understood by means of the Bethe ansatz [70-72] and conformal field theory [73-75]. The solutions established a novel concept of the Tomonaga-Luttinger liquid [76] which is described by the scalar bosons corresponding to charge and spin sectors, respectively. The correlated electrons in two- and three-dimensional space are still far from a complete understanding in spite of the success for the one-dimensional Hubbard model. A possibility of superconductivity is a hot topic as well as the magnetism and metal-insulator transition for the two- and three-dimensional Hubbard model.

The three-band Hubbard model that contains $d$ and $p$ orbitals has also been investigated intensively with respect to high temperature superconductors [24,64, $77-88]$. This model is also called the $d-p$ model. The 2D three-band Hubbard model is the more realistic and relevant model for two-dimensional $\mathrm{CuO}_{2}$ planes which are contained usually in the crystal structures of high- $T_{c}$ superconductors. The network of $\mathrm{CuO}_{2}$ layer is shown in Figure 3. The parameters of the three-band Hubbard model are given by the Coulomb repulsion $U_{d}$, energy levels of $p$ electrons $\epsilon_{p}$ and $d$ electron $\epsilon_{d}$, and transfer between $p$ orbitals given by $t_{p p}$. Typical parameter values for the three-band $(d-p)$ Hubbard model are shown in Table 1. The Hamiltonian of the 


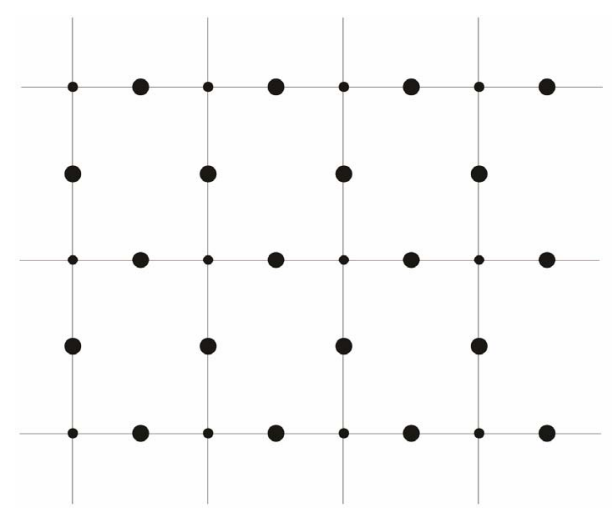

Figure 3. The lattice of the three-band Hubbard model on the $\mathrm{CuO}_{2}$ plane. Small circles denote $\mathrm{Cu}$ sites and large ones denote $\mathrm{O}$ sites.

Table 1. Typical parameter values for the three-band Hubbard model. Energies are measured in $\mathrm{eV}$.

\begin{tabular}{ccccc}
\hline & Ref.[90] & Ref.[89] & Ref.[91] & Ref.[78] \\
\hline$\epsilon_{p}-\epsilon_{d}$ & 3.6 & $2.75-3.75$ & 3.5 & 2.5 \\
$t_{d p}$ & 1.3 & 1.5 & 1.5 & 1.47 \\
$t_{p p}$ & 0.65 & 0.65 & 0.6 & \\
$U_{d}$ & 10.5 & 8.8 & 9.4 & 9.7 \\
$U_{p}$ & 4.0 & 6.0 & 4.7 & 5.7 \\
$U_{d p}$ & 1.2 & $<1.0$ & 0.8 & $<1$ \\
\hline
\end{tabular}

three-band Hubbard model is written as [24,47,48,80] (see Equation (4)) where $\hat{x}$ and $\hat{y}$ represent unit vectors along $x$ and $y$ directions, respectively. $p_{i \pm \hat{x} / 2, \sigma}^{\dagger}$ and $p_{i \pm \hat{x} / 2, \sigma}$ denote the operators for the $p$ electrons at site $R_{i} \pm \hat{x} / 2$. Similarly $p_{i \pm \hat{y} / 2, \sigma}^{\dagger}$ and $p_{i \pm \hat{y} / 2, \sigma}$ are defined. $U_{d}$ denotes the strength of Coulomb interaction between $d$ electrons. For simplicity we neglect the Coulomb interaction among $p$ electrons in this paper. Other notations are standard and energies are measured in $t_{d p}$ units. The number of cells is denoted as $N$ for the three-band Hubbard model. In the non-interacting case $\left(U_{d}=0\right)$ the Hamiltonian in the $k$-space is written as (see Equation (5)). where $d_{\boldsymbol{k} \sigma}\left(d_{\boldsymbol{k} \sigma}^{\dagger}\right), p_{x \boldsymbol{k} \sigma}\left(p_{x \boldsymbol{k} \sigma}^{\dagger}\right)$ and $p_{y \boldsymbol{k} \sigma}\left(p_{y \mathbf{k} \sigma}^{\dagger}\right)$ are operators for $d-, p_{x}$ - and $p_{y}$-electron of the momentum $\boldsymbol{k}$ and spin $\sigma$, respectively.

In the limit $t_{d p} \ll U_{d}-\left(\epsilon_{p}-\epsilon_{d}\right), t_{d p} \ll \epsilon_{p}-\epsilon_{d}$, and $\epsilon_{p}-\epsilon_{d}<U_{d}$, the $d-p$ model is mapped to the $t-J$ model with

$$
J=4 t_{e f f}^{2}\left(\frac{1}{U_{d}}+\frac{2}{2\left(\epsilon_{p}-\epsilon_{d}\right)+U_{p}}\right),
$$

where $t_{\text {eff }} \simeq t_{d p}^{2} /\left(\epsilon_{p}-\epsilon_{d}\right) . \quad J_{K}=4 t_{\text {eff }}$ gives the antiferromagnetic coupling between the neighboring $d$ and $p$ electrons. In real materials $\left(\epsilon_{p}-\epsilon_{d}\right) / t_{d p}$ is not so large. Thus it seems that the mapping to the $t-J$ model is not necessarily justified.

\section{Variational Monte Carlo Studies}

In this Section we present studies on the two-dimensional Hubbard model by using the variational Monte Carlo method.

\subsection{Variational Monte Carlo Method}

Let us start by describing the method based on the 2D Hubbard model. The Hamiltonian is given by

$$
\begin{aligned}
H= & -t \sum_{\langle i j\rangle \sigma}\left(c_{i \sigma}^{\dagger} c_{j \sigma}+\text { h.c. }\right) \\
& -t^{\prime} \sum_{\langle\langle j \ell\rangle\rangle \sigma}\left(c_{j \sigma}^{\dagger} c_{\ell \sigma}+\text { h.c. }\right)+U \sum_{j} n_{j \uparrow} n_{j \downarrow},
\end{aligned}
$$

where $\langle i j\rangle$ denotes summation over all the nearestneighbor bonds and $\langle\langle j \ell\rangle\rangle$ means summation over the next nearest-neighbor pairs. $t$ is our energy unit. The dispersion is given by

$$
\epsilon_{\mathbf{k}}=-2 t\left(\cos \left(k_{x}\right)+\cos \left(k_{y}\right)\right)-4 t^{\prime} \cos \left(k_{x}\right) \cos \left(k_{y}\right) .
$$

Our trial wave function is the Gutzwiller-projected wave functions defined as

$$
\begin{gathered}
\psi_{n}=P_{N_{e}} P_{G} \psi_{0}, \\
\psi_{s}=P_{N_{e}} P_{G} \psi_{B C S},
\end{gathered}
$$

$$
\begin{aligned}
H_{d p}= & \epsilon_{d} \sum_{i \sigma} d_{i \sigma}^{\dagger} d_{i \sigma}+U_{d} \sum_{i} d_{i \uparrow}^{\dagger} d_{i \uparrow} d_{i \downarrow}^{\dagger} d_{i \downarrow}+\epsilon_{p} \sum_{i \sigma}\left(p_{i+\hat{x} / 2, \sigma}^{\dagger} p_{i+\hat{x} / 2, \sigma}+p_{i+\hat{y} / 2, \sigma}^{\dagger} p_{i+\hat{y} / 2, \sigma}\right) \\
& -t_{d p} \sum_{i \sigma}\left[d_{i \sigma}^{\dagger}\left(p_{i+\hat{x} / 2, \sigma}+p_{i+\hat{y} / 2, \sigma}-p_{i-\hat{x} / 2, \sigma}-p_{i-\hat{y} / 2, \sigma}\right)+\text { h.c. }\right] \\
& -t_{p p} \sum_{i \sigma}\left[p_{i+\hat{y} / 2, \sigma}^{\dagger} p_{i+\hat{x} / 2, \sigma}-p_{i+\hat{y} / 2, \sigma}^{\dagger} p_{i-\hat{x} / 2, \sigma}-p_{i-\hat{y} / 2, \sigma}^{\dagger} p_{i+\hat{x} / 2, \sigma}+p_{i-\hat{y} / 2, \sigma}^{\dagger} p_{i-\hat{x} / 2, \sigma}+\text { h.c. }\right] . \\
H_{d p}^{0}= & \epsilon_{d} \sum_{\boldsymbol{k} \sigma} d_{\boldsymbol{k} \sigma}^{?} d_{\boldsymbol{k} \sigma}+\epsilon_{p} \sum_{\boldsymbol{k} \sigma}\left(p_{x \boldsymbol{k} \sigma} p_{x \boldsymbol{k} \sigma}+p_{y \mathbf{k} \sigma} p_{y \boldsymbol{k} \sigma}\right)+\sum_{\boldsymbol{k} \sigma}\left(2 \mathrm{i} t_{d p} \sin \left(k_{x} / 2\right) d_{\boldsymbol{k} \sigma} p_{x \boldsymbol{k} \sigma}+\text { h.c. }\right) \\
& +\sum_{\mathbf{k} \sigma}\left(2 \mathrm{i} t_{d p} \sin \left(k_{y} / 2\right) d_{\boldsymbol{k} \sigma}^{\dagger} p_{y \mathbf{k} \sigma}+\text { h.c. }\right)+\sum_{\mathbf{k} \sigma}\left(-4 t_{p p} \sin \left(k_{x} / 2\right) \sin \left(k_{y} / 2\right)\right)\left(p_{x \boldsymbol{k} \sigma}^{\dagger} p_{y \mathbf{k} \sigma}+\text { h.c. }\right),
\end{aligned}
$$


where

$$
\begin{gathered}
\psi_{0}=\prod_{|\boldsymbol{k}| \leq k_{F}, \sigma} c_{\boldsymbol{k} \sigma}^{\dagger}|0\rangle, \\
\psi_{B C S}=\prod_{\boldsymbol{k}}\left(u_{\boldsymbol{k}}+v_{\boldsymbol{k}} c_{\boldsymbol{k} \uparrow}^{\dagger} c_{-\boldsymbol{k} \downarrow}^{\dagger}\right)|0\rangle .
\end{gathered}
$$

$P_{G}$ is the Gutzwiller projection operator given by

$$
P_{G}=\prod_{k}\left[1-(1-g) n_{j \uparrow} n_{j \downarrow}\right] ;
$$

$g$ is a variational parameter in the range from 0 to unity and $j$ labels a site in the real space. $P_{N_{e}}$ is a projection operator which extracts only the sites with a fixed total electron number $N_{e}$. Coefficients $u_{\boldsymbol{k}}$ and $v_{\boldsymbol{k}}$ in $\psi_{B C S}$ appear in the ratio defined by

$$
\frac{v_{k}}{u_{k}}=\frac{\Delta_{k}}{\xi_{k}+\left(\xi_{k}^{2}+\Delta_{k}^{2}\right)^{1 / 2}},
$$

where $\xi_{k}=\varepsilon_{k}-\mu$ and $\Delta_{k}$ is a $k$-dependent gap function. $\mu$ is a variational parameter working like the chemical potential. $c_{k \sigma}$ is the Fourier transform of $c_{j \sigma}$. The wave functions $\psi_{n}$ and $\psi_{s}$ are expressed by the Slater determinants for which the expectations values are evaluated using a Monte Carlo procedure $[43,44,92] . \psi_{s}$ is written as

$$
\begin{aligned}
\psi_{s} & \propto P_{N_{e}} P_{G} \exp \left[\sum_{\boldsymbol{k}}\left(v_{\boldsymbol{k}} / u_{\boldsymbol{k}}\right) c_{\boldsymbol{k} \uparrow}^{\dagger} \uparrow_{-\boldsymbol{k} \downarrow}^{\dagger}\right]|0\rangle \\
& =P_{N_{e}} P_{G} \exp \left[\sum_{j \ell} a(j, \ell) c_{j \uparrow}^{\dagger} \uparrow_{\ell \downarrow}^{\dagger}\right]|0\rangle \\
& \propto P_{G}\left[\sum_{j \ell} a(j, \ell) c_{j \uparrow}^{\dagger} c_{\ell \downarrow}^{\dagger}\right]^{N_{e} / 2}|0\rangle,
\end{aligned}
$$

where

$$
a(j, \ell)=(1 / N) \sum_{\boldsymbol{k}}\left(v_{\boldsymbol{k}} / u_{\boldsymbol{k}}\right) \exp \left[i \boldsymbol{k} \cdot\left(\boldsymbol{R}_{\ell}-\boldsymbol{R}_{j}\right)\right] .
$$

Then $\psi_{s}$ is written using the Slater determinants as

$$
\begin{aligned}
& \psi_{s}=P_{G} \sum_{j_{1} \cdots j_{N_{e} / 2} \ell_{1} \cdots \ell_{N_{e} / 2}} A\left(j_{1} \cdots i_{N_{e} / 2}, \ell_{1} \cdots \ell_{N_{e} / 2}\right) \\
& \times c_{j_{1} \uparrow}^{\dagger} c_{j_{2} \uparrow}^{\dagger} \cdots c_{j_{N_{e} / 2}^{\dagger} \uparrow}^{\dagger} c_{\ell_{1} \downarrow}^{\dagger} \downarrow c_{\ell_{2} \downarrow}^{\dagger} \cdots c_{\ell_{N_{e} / 2} \downarrow}^{\dagger}|0\rangle,
\end{aligned}
$$

where $A\left(j_{1} \cdots i_{N_{e} / 2}, \ell_{1} \cdots \ell_{N_{e} / 2}\right)$ is the Slater determinant defined by

$$
\begin{aligned}
& A\left(j_{1} \cdots \ell_{N_{e} / 2}\right) \\
& =\left|\begin{array}{cccc}
a\left(j_{1}, \ell_{1}\right) & a\left(j_{1}, \ell_{2}\right) & \cdots & a\left(j_{1}, \ell_{N_{e} / 2}\right) \\
a\left(j_{2}, \ell_{1}\right) & a\left(j_{2}, \ell_{2}\right) & \cdots & a\left(j_{2}, \ell_{N_{e} / 2}\right) \\
\vdots & \vdots & \ddots & \vdots \\
a\left(j_{N_{e} / 2}, \ell_{1}\right) & a\left(j_{N_{e} / 2}, \ell_{2}\right) & \cdots & a\left(j_{N_{e} / 2}, \ell_{N_{e} / 2}\right)
\end{array}\right| .
\end{aligned}
$$

In the process of Monte Carlo procedure the values of cofactors of the matrix in Equation (18) are stored and corrected at each time when the electron distribution is modified. We optimized the ground state energy

$$
E_{g}=\langle H\rangle=\left\langle\psi_{s}|H| \psi_{s}\right\rangle /\left\langle\psi_{s} \mid \psi_{s}\right\rangle
$$

with respect to $g, \Delta_{\boldsymbol{k}}$ and $\mu$ for $\psi_{s}$ for $\psi_{s}$. For $\psi_{n}$ the variational parameter is only $g$. We can employ the correlated measurements method [93] in the process of searching optimal parameter values minimizing $E_{g}$.

A Monte Carlo algorithm developed in the auxiliary field quantum Monte Carlo calculations can also be employed in evaluating the expectation values for the wave functions shown above [94-96]. Note that the Gutzwiller projection operator is written as

$$
P_{G}=\exp \left(-\alpha \sum_{i} n_{i \uparrow} n_{i \downarrow}\right),
$$

where $\alpha=\log (1 / g)$. Then using the discrete HubbardStratonovich transformation, the Gutzwiller operator is the bilinear form:

$$
\begin{aligned}
& \exp \left(-\alpha \sum_{i} n_{i \uparrow} n_{i \downarrow}\right) \\
& =(1 / 2)^{N} \sum_{\left\{s_{i}\right\}} \exp \left[2 a \times \sum_{i} s_{i}\left(n_{i \uparrow}-n_{i \downarrow}\right)-\frac{\alpha}{2} \sum_{i}\left(n_{i \uparrow}+n_{i \downarrow}\right)\right],
\end{aligned}
$$

where $\cosh (2 a)=\mathrm{e}^{\alpha / 2}$. The Hubbard-Stratonovich auxiliary field $s_{i}$ takes the values of \pm 1 . The norm $\left\langle\psi_{n} \mid \psi_{n}\right\rangle$ is written as

$$
\begin{aligned}
\left\langle\psi_{n} \mid \psi_{n}\right\rangle= & \text { const } \cdot \sum_{\left\{u_{i}\right\}\left\{s_{i}\right\}} \prod_{\sigma} \operatorname{det}\left(\phi_{0}^{\sigma \dagger} \exp \left(V^{\sigma}(u, \alpha)\right)\right. \\
& \left.\times \exp \left(V^{\sigma}(s, \alpha)\right) \phi_{0}^{\sigma}\right),
\end{aligned}
$$

where $V^{\sigma}(s, \alpha)$ is a diagonal $N \times N$ matrix corresponding to the potential

$$
\begin{gathered}
h^{\sigma}(s)=2 a \sigma \sum_{i} s_{i} n_{i \sigma}-\frac{\alpha}{2} \sum_{i} n_{i \sigma} . \\
V^{\sigma}(s, \alpha) \text { is written as } \\
V^{\sigma}(s, \alpha)=\operatorname{diag}\left(2 a \sigma s_{1}-\alpha / 2, \cdots, 2 a \sigma s_{N}-\alpha / 2,0, \cdots\right),
\end{gathered}
$$

where $\operatorname{diag}(a, \cdots)$ denotes a diagonal matrix with elements given by the arguments $a, \cdots$. The elements of $\left(\phi_{0}^{\sigma}\right)_{i j} \quad\left(i=1, \cdots, N ; j=1, \cdots, N_{e} / 2\right)$ are given by linear combinations of plane waves. For example,

$$
\left(\phi_{0}^{\sigma}\right)_{i j}=\exp \left(i \boldsymbol{r}_{i} \cdot \boldsymbol{k}_{j}\right) .
$$

Then we can apply the standard Monte Carlo sampling method to evaluate the expectation values $[94,95]$. This 
method is used to consider an off-diagonal Jastrow correlation factor of $\exp (-S)$-type. The results for the improved wave functions are discussed in Section 3.10.

\subsection{Superconducting Condensation Energy}

We study the cases of the $d$-, extended $s-\left(s^{*}-\right)$ and $s$-wave gap functions in the following:

$$
\begin{gathered}
d \quad \Delta_{\boldsymbol{k}}=\Delta\left(\cos \left(k_{x}\right)-\cos \left(k_{y}\right)\right), \\
s^{*} \Delta_{\boldsymbol{k}}=\Delta\left(\cos \left(k_{x}\right)+\cos \left(k_{y}\right)\right), \\
s \quad \Delta_{\boldsymbol{k}}=\Delta .
\end{gathered}
$$

In Figure 4 calculated energies per site with $N_{e}=84$ on the $10 \times 10$ lattice are shown for the case of $U=8$ and $t^{\prime}=0$ [46]. $E_{g} / N$ is plotted as a function of $\Delta$ for three types of gap functions shown above. We impose the periodic and the antiperiodic boundary conditions for $x$ - and $y$-direction, respectively. This set of boundary conditions is chosen so that $\Delta_{k}$ does not vanish for any $k$-points occupied by electrons. $E_{g}$ was obtained as the average of the results of several Monte Carlo calculations each with $5 \times 10^{7}$ steps. $E_{g} / N$ has minimum at a finite value of $\Delta \simeq 0.08$ in the case of the $d$-wave gap function.

The energy gain $\Delta E_{g}$ in the superconducting state is called the SC condensation energy in this paper. $\Delta E_{g} / N$ is plotted as a function of $1 / N$ in Figure 5 in order to examine the size dependence of the SC energy gain [97]. Lattice sizes treated are from $8 \times 8$ to $22 \times 22$. The electron density $n_{e}$ is in the range of $0.80 \geq n_{e} \leq 0.86$. Other parameters are $-0.20 \leq t^{\prime} \leq 0.0$ and $U / t=8$ in $t$ units. Bulk limit $N \rightarrow \infty$ of SC condensation energy $E_{\text {cond }}$ was obtained by plotting as a function of $1 / N$. The linear fitting line indicates very

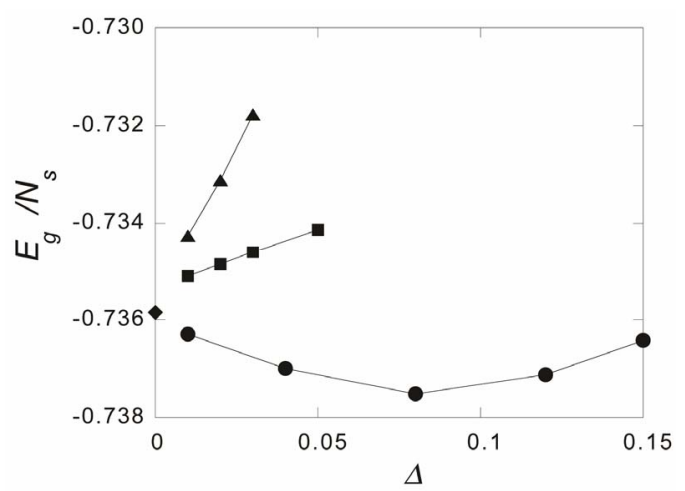

Figure 4. Ground state energy per site $E_{g} / N$ for the 2D

Hubbard model is plotted against $\Delta$ for the case of 84 electrons on the $10 \times 10$ lattice with $U=8$ and $t^{\prime}=0$. Solid curves are for the $d$-wave gap function. Squares and triangles are for the $s^{*}$ - and $s$-wave gap functions, respectively. The diamond shows the normal state value [46].

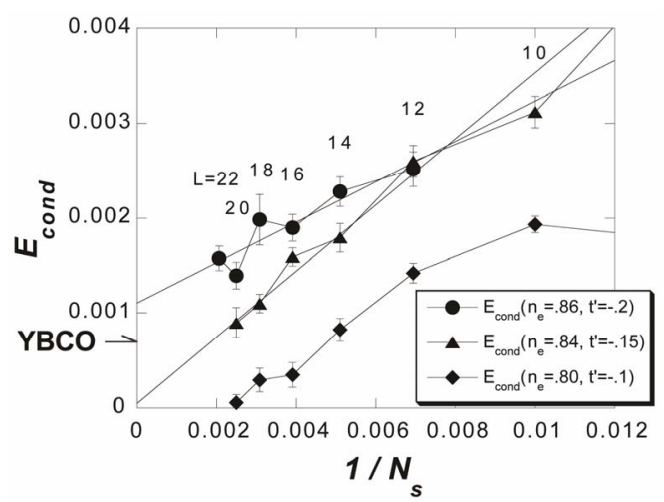

Figure 5. Energy gain per site in the SC state with reference to the normal state for the 2D Hubbard model is plotted as a function of $1 / N . L$ is the length of the edge of the square lattice. YBCO attached to the vertical axis indicates the experimental value of the SC condensation energy for $\mathrm{YBa}_{2} \mathrm{Cu}_{3} \mathrm{O}_{4}$ [97].

clearly that the bulk limit remains finite when $-0.25 \leq t^{\prime} \leq-0.10$ and $n_{e} \geq 0.84$. When $n_{e}=0.86$, $t^{\prime}=-0.20$ and $U=8$, the bulk-limit $E_{\text {cond }}$ is $E_{\text {cond }}=0.00117 / \mathrm{site} \simeq 0.60 \mathrm{meV} / \mathrm{site}$, where $t=0.51$ $\mathrm{eV}$ is used [98]. Thus the superconductivity is a real bulk property, not a spurious size effect. The value is remarkably close to experimental values $0.17 \sim 0.26$ $\mathrm{meV} /$ site estimated from specific heat data $[99,100]$ and $0.26 \mathrm{meV} /$ site from the critical magnetic field $H_{c}$ [101] for optimally doped $\mathrm{YBa}_{2} \mathrm{Cu}_{3} \mathrm{O}_{4}$ (YBCO). This good agreement strongly indicates that the 2D Hubbard model includes essential ingredients for the superconductivity in the cuprates.

We just point out that the $t-J$ model gives $E_{\text {cond }}=0.026 t \simeq 13 \mathrm{meV} / \mathrm{site}$ at $n_{e}=0.84$ for $J=4$ $t^{2} / U=0.5$ and $t^{\prime}=0$ [102]. This value is 50 times larger than the experimental values indicating a serious quantitative problem with this model. This means that the $t-J$ model made from the leading two terms in the expansion in terms of $t / U$ of the canonical transformation of the Hubbard model should be treated with the higher-order terms in order to give a realistic SC condensation energy.

Here we show the SC condensation energy as a function of $U$ in Figure 6. The condensation energy $E_{\text {cond }}=\Delta E / N$ is increased as $U / t$ is increased as far as $U / t \leq 12$. In the strong coupling region $U>8 t$, we obtain the large condensation energy.

\subsection{Fermi Surface and Condensation Energy}

Now let us consider the relationship between the Fermi surface structure and the strength of superconductivity. The experimental SC condensation energy for $(\mathrm{La}, \mathrm{Sr})_{2} \mathrm{CuO}_{4}(\mathrm{LSCO})$ is estimated at $0.029 \mathrm{meV} /(\mathrm{Cu}$ site $)$ or 0.00008 in units of $t$ which is much smaller than that 


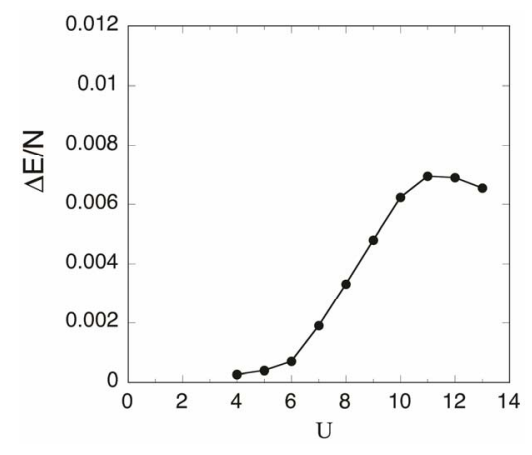

Figure 6. Energy gain per site in the SC state with reference to the normal state for the $2 \mathrm{D}$ Hubbard model as a function of the Coulomb repulsion $U$. The system is $10 \times 10$ with the electron number $N_{e}=84$ and $t^{\prime}=-0.3$.

for YBCO. [103] The band parameter values of LSCO were estimated as $t^{\prime}=-0.12$ and $t^{\prime \prime}=0.08$ [104]. This set corresponds roughly to $E_{\text {cond }} \simeq 0.0010$. The latter value is much larger than the above-mentioned experimental value for LSCO. However, the stripe-type SDW state coexists with superconductivity $[105,106]$ and the SC part of the whole $E_{\text {cond }}$ is much reduced. Therefore, such a coexistence allows us to qualitatively understand the SC $E_{\text {cond }}$ in LSCO.

On the other hand, T12201 $\left(T_{c}=93 \mathrm{~K}\right)$ and $\mathrm{Hg} 1201$ $\left(T_{c}=98 \mathrm{~K}\right)$ band calculations by Singh and Pickett [107] give very much deformed Fermi surfaces that can be fitted by large $\left|t^{\prime}\right|$ such as $t^{\prime} \sim-0.4$. For T12201, an Angular Magnetoresistance Oscillations (AMRO) work [108] gives information of the Fermi surface, which allows to get $t^{\prime} \sim-0.2$ and $t^{\prime \prime} \sim 0.165$. There is also an Angle-Resolved Photoemission Study (ARPES) [109], which provides similar values. In the case of $\mathrm{Hg} 1201$, there is an ARPES work [110], form which we obtain by fitting $t^{\prime} \sim-0.2$ and $t^{\prime \prime} \sim 0.175$. For such a deformed Fermi surface, $E_{\text {cond }}$ in the bulk limit is reduced considerably. [111,112] Therefore, the SC $E_{\text {cond }}$ calculated by VMC indicates that the Fermi surface of LSCO-type is more favorable for high $T_{c}$. The lower $T_{c}$ in LSCO may be attributed to the coexistence with antiferromagnetism of stripe type.

\subsection{Ladder Hubbard Model}

The SC condensation energy in the bulk limit for the ladder Hubbard model has also been evaluated using the variational Monte Carlo method [54]. The Hamiltonian is given by the 1D two-chain Hubbard model: [51,52,55, $56,85,113-116]$

$$
\begin{aligned}
H_{\text {ladder }}= & -t_{d} \sum_{\ell \sigma}\left(c_{l \ell \sigma}^{\dagger} c_{2 \ell \sigma}+\text { h.c. }\right)-t \sum_{j=1}^{2} \sum_{\ell \sigma}\left(c_{j \ell \sigma}^{\dagger} c_{j, \ell+1, \sigma}+\text { h.c. }\right) \\
& +U_{0} \sum_{j=1}^{2} \sum_{\ell} c_{j \ell \uparrow}^{\dagger} c_{j \ell \uparrow} c_{j \ell \downarrow}^{\dagger} c_{j \ell \downarrow},
\end{aligned}
$$

where $c_{j \ell \sigma}^{\dagger}\left(c_{j \ell \sigma}\right)$ is the creation (annihilation) operator of an electron with spin $\sigma$ at the $\ell$ th site along the $j$ th chain $(j=1,2) . t$ is the intrachain nearest-neighbor transfer and $t_{d}$ is the interchain nearest-neighbor transfer energy. The energy is measured in $t$ units. The energy minimum was given when the components of the gap function $\Delta_{k}$ take finite values plotted in Figure 7 for the lattice of $20 \times 2$ sites with 34 electrons imposing the periodic boundary condition [54]. Each component of $\Delta_{k}$ was optimized for $U_{0}=8$ and $t_{d}=1.8$. There are two characteristic features; one is that the components of the bonding and antibonding bands have opposite signs each other and second is that the absolute values of $\Delta_{k}$ of the antibonding band $\left(k_{y}=\pi\right)$ is much larger than that of the bonding band $\left(k_{y}=0\right)$. In order to reduce the computation cpu time, $\Delta_{k}$ of each band was forced to take a fixed value specific to each band, i.e. $\Delta_{1}$ for the bonding band and $\Delta_{2}$ for the antibonding band. This drastically reduces the number of the variational parameters but still allows us to get a substantial value of the condensation energy. $\Delta_{1}$ and $\Delta_{2}$ take opposite sign, which is similar to that of the $d_{x^{2}-y^{2}}$ gap function.

The energy gain $\Delta F_{2 c}$ remains finite in the bulk limit when $1.2<t_{d}<1.6$. The SC condensation energy per site in the bulk limit is plotted as a function of $t_{d}$ in Figure 8 [54]. The SC region derived from the SC condensation energy in the bulk limit is consistent with the results obtained from the density-matrix renormalization group $[55,56]$ and the exact-diagonalization method [51,52,115]. The maximum value of $\Delta E_{2 c}$ is 0.0008 which is of the same order of magnitude as the maximum condensation energy obtained for the 2D Hubbard model [46].

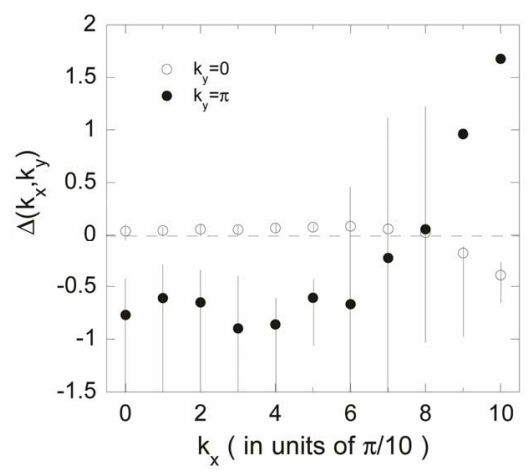

Figure 7. The values of components of $\Delta_{k}$ for the twochain Hubbard model. All the values of $k_{x}$ of the bonding band $\left(k_{y}=0\right)$ and antibonding band $\left(k_{y}=\pi\right)$ correspond to the energy minimum for $20 \times 2$ lattice with 34 electrons. The parameters in the Hamiltonian are $t_{d}=1.8$ and $U_{0}=8$ and the variational parameters are $\mu=0.0182$ and $g=0.415$ [54]. 


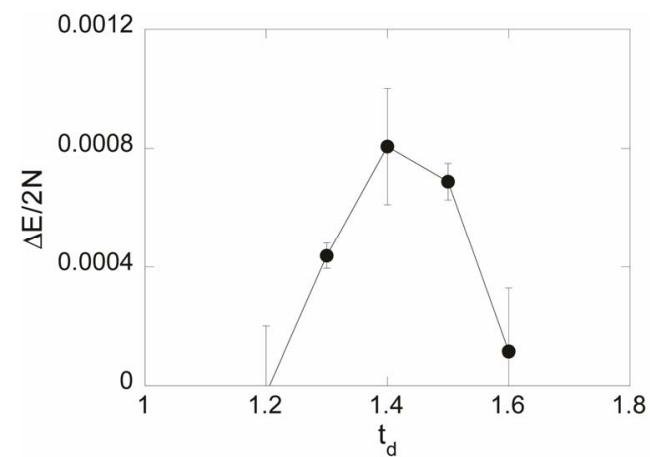

Figure 8. $t_{d}$ dependence of the SC condensation energy $\Delta E / 2 N$ for the two-chain Hubbard model in the bulk limit [54].

\subsection{Condensation Energy in the $d-p$ Model}

The SC energy gain for the $d-p$ model, namely, threeband Hubbard model in Equation (4) has also been evaluated using the variational Monte Carlo method. For the three-band model the wave functions are written as

$$
\begin{gathered}
\psi_{n}=P_{G} \prod_{|k| \leq k_{F}, \sigma} \alpha_{k \sigma}^{\dagger}|0\rangle, \\
\psi_{S C}=P_{G} P_{N_{e}} \prod_{k}\left(u_{k}+v_{k} \alpha_{k \uparrow}^{\dagger} \alpha_{-k \downarrow}^{\dagger}\right)|0\rangle,
\end{gathered}
$$

where $\alpha_{k \sigma}$ is the linear combination of $d_{k \sigma}, p_{x k \sigma}$ and $p_{y k \sigma}$ constructed to express the operator for the lowest band (in the hole picture) or the highest band (in the electron picture) of the non-interacting Hamiltonian. The numerical calculations have been done in the hole picture. The Gutzwiller parameter $g$, effective level difference $\tilde{\epsilon}_{p}-\tilde{\epsilon}_{d}$, chemical potential $\mu$ and superconducting order parameter $\Delta$ are the variational parameters.

The similar results to the single-band Hubbard model were obtained as shown in Figure 9 for $t_{p p}=0.0$, $U_{d}=8$ and $\epsilon_{d}-\epsilon_{p}=2$ in $t_{d p}$ units where the calculations were performed in the hole picture [24]. The SC condensation energy for the three-band model is $E_{\text {cond }} \simeq 0.0005 t_{d p} \simeq 0.75 \mathrm{meV}$ per site in the optimally doped region. We set $t_{d p}=1.5 \mathrm{eV}$ as estimated in Table 1. There is a tendency that $E_{\text {cond }}$ increases as $\epsilon_{d}-\epsilon_{p}$ increases which is plotted in Figure 10. This tendency is not, however, in accordance with NQR (nuclear quadrupole resonance) study on cuprates. [117] We think that the NQR experiments indicate an importance of the Coulomb interaction on oxygen sites. This will be discussed in Section 3.11.

\subsection{Antiferromagnetic State}

When the density of doped holes is zero or small, the 2D single-band or three-band Hubbard model takes an antiferromganetic state as its ground state. The magnetic
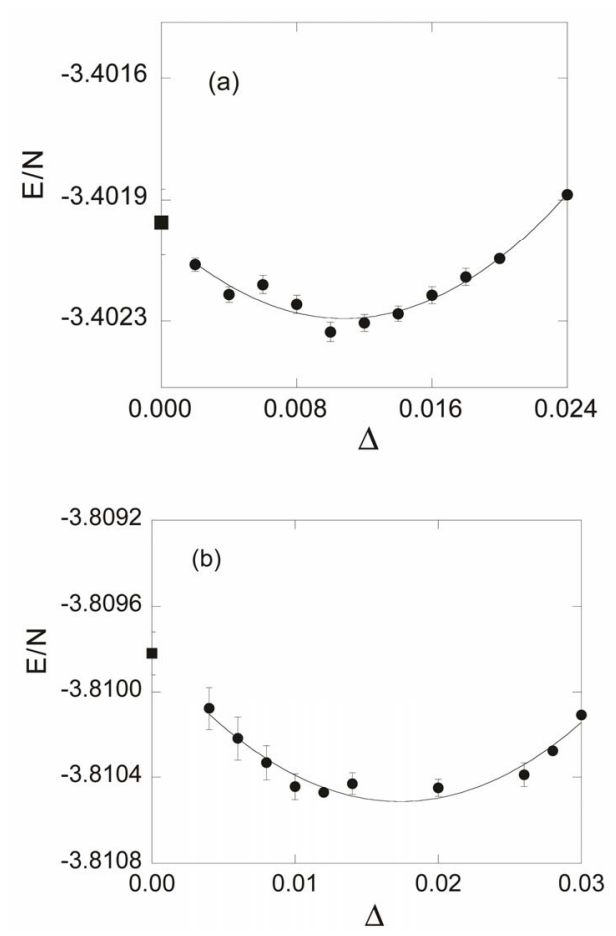

Figure 9. Ground-state energy per site as a function of $\Delta$ with the $d$-wave gap function for the three-band Hubbard model. The size of lattice is $6 \times 6$. Parameters are $U=8$, $t_{p p}=0$ and $\epsilon_{p}-\epsilon_{d}=2$ in units of $t_{d p}$. The doping rate is $\delta=0.111$ for (a) and $\delta=0.333$ for (b). Squares denote the energies for the normal-state wave function [24].

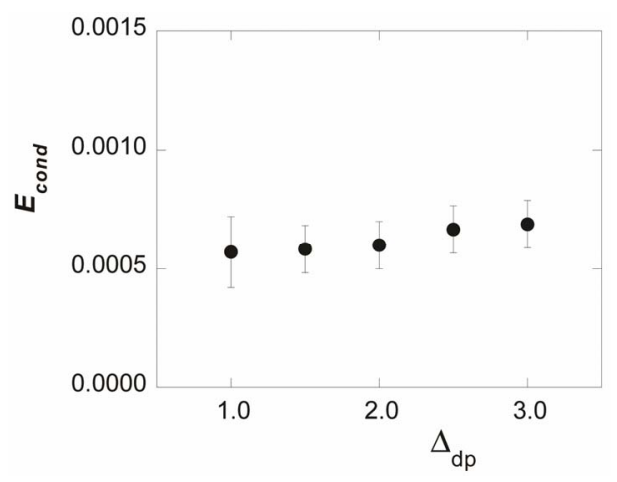

Figure 10. Energy gain per site in the SC state as a function of the level difference $\Delta_{d p}=\epsilon_{p}-\epsilon_{d}$ for the three-band Hubbard model with $U_{d}=8$ and $t_{p p}=0.2$ [24]. The size of lattice is $6 \times 6$ sites.

order is destroyed and superconductivity appears with the increase of doped hole density. The transition between the $d$-wave SC and the uniform SDW states has been investigated by computing the energy of the SDW state by using the variational Monte Carlo method. The trial SDW wave function is written as

$$
\psi_{A F}=P_{G} \psi_{S D W},
$$




$$
\begin{gathered}
\psi_{S D W}=\prod_{\boldsymbol{k}}\left(u_{\boldsymbol{k}} c_{\boldsymbol{k} \uparrow}^{\dagger}+v_{\boldsymbol{k}} c_{\boldsymbol{k}+Q \uparrow}^{\dagger}\right) \times \prod_{\boldsymbol{k}^{\prime}}\left(u_{\boldsymbol{k}^{\prime}} c_{\boldsymbol{k}^{\prime} \downarrow}^{\dagger}-v_{\boldsymbol{k}^{\prime}} c_{\boldsymbol{k}^{\prime}+Q \downarrow}^{\dagger}\right)|0\rangle, \\
u_{\boldsymbol{k}}=\left[\left(1-w_{\boldsymbol{k}} /\left(w_{\boldsymbol{k}}^{2}+\Delta_{A F}^{2}\right)^{1 / 2}\right) / 2\right]^{1 / 2}, \\
v_{\boldsymbol{k}}=\left[\left(1+w_{\boldsymbol{k}} /\left(w_{\boldsymbol{k}}^{2}+\Delta_{A F}^{2}\right)^{1 / 2}\right) / 2\right]^{1 / 2} \\
w_{k}=\left(\epsilon_{\boldsymbol{k}}-\epsilon_{\boldsymbol{k}+Q}\right) / 2
\end{gathered}
$$

Summation over $\boldsymbol{k}$ and $\boldsymbol{k}^{\prime}$ in Equation (33) is performed over the filled $k$-points, as in the calculation of the normal state energy. $Q$ is the SDW wave vector given by $(\pi, \pi)$ and $\Delta_{A F}$ is the SDW potential amplitude.

As shown in Figure 11, the energy gain per site in the SDW state rises very sharply from $n_{e} \sim 0.84$ for $t^{\prime}=0$ [46]. At $n_{e} \sim 0.84$ it is slightly larger than that in the SC state, and at $n_{e}=0.80$ there is no more stable SDW state. Thus the boundary between the SDW and the SC states is given at $n_{e} \sim 0.84$. The results of the bulk limit calculations indicate that the energy gain in the SC state at $n_{e}=0.84$ takes the extremely small value and the value at $n_{e}=0.80$ vanishes as $N \rightarrow 0$. Hence the pure $d$-wave SC state possibly exists near the boundary at $n_{e} \sim 0.84$, but the region of pure SC state is very restricted.

Let us turn to the three-band model. We show the antiferromagnetic-paramagnetic boundary for $t_{p p}=0.0$ and $\epsilon_{p}-\epsilon_{d}=2$ in the plane of $U$ and the hole density in Figure 12 where AF denotes the antiferromagnetic region [47]. The value $\epsilon_{p}-\epsilon_{d}=2$ is taken from the estimations by cluster calculations [89-91]. The phase boundary in the region of small $U$ is drawn from an extrapolation. For the intermediate values of $U \sim 8-12$,

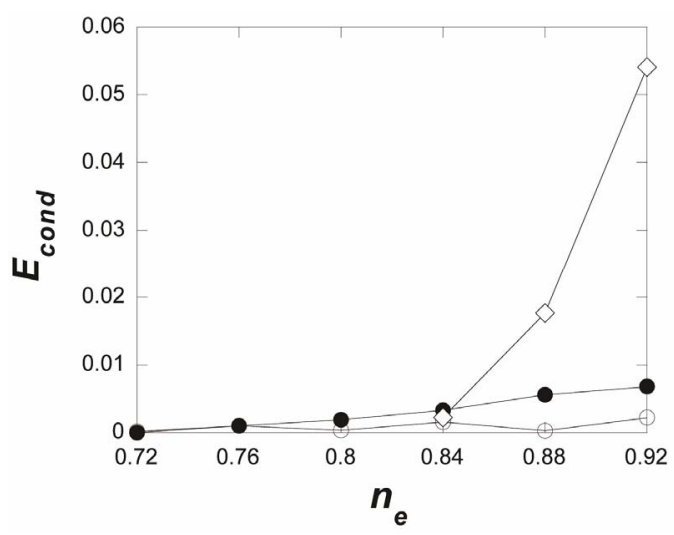

Figure 11. Energy gain per site in the SDW state (diamonds) against electron density for $t^{\prime}=0$ and the energy gain in the SC state for $t^{\prime}=0$ (open circles) and $t^{\prime}=-0.1$ (solid circles). The model is the $2 \mathrm{D}$ Hubbard model on $10 \times 10$ lattice [46].

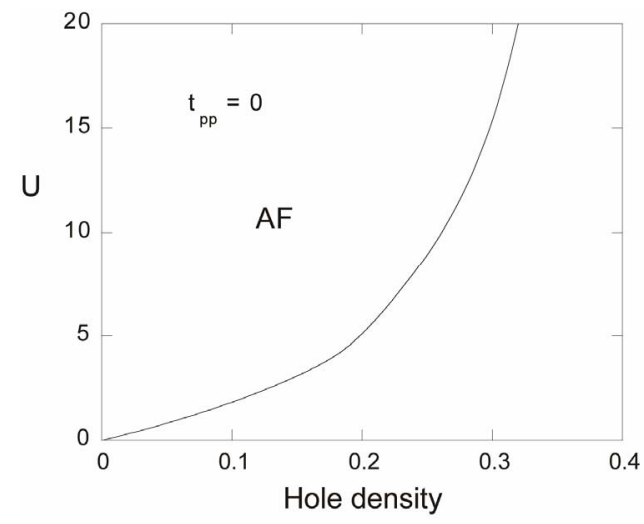

Figure 12. Antiferromagnetic region in the plane of $U$ and the hole density for $t_{p p}=0.0$ and $\epsilon_{p}-\epsilon_{d}=2$.

the antiferromagnetic long-range ordering exists up to about 20 percent doping. Thus the similar features are observed compared to the single-band Hubbard model.

Since the three-band Hubbard model contains several parameters, we must examine the parameter dependence of the energy of SDW state. The energy gain $\Delta E_{S D W}$ in the SDW state is shown in Figure 13 as a function of doping ratio for several values of $\Delta_{d p} \equiv \epsilon_{p}-\epsilon_{d} . \Delta E_{S D W}$ increases as $\Delta_{d p}$ increases as expected. In Figure 14 $t_{p p}$ - and $U_{d}$-dependencies of $\Delta E_{S D W}$ are presented. The SDW phase extends up to 30 percent doping when $U_{d}$ is large. It follows from the calculations that the SDW region will be reduced if $\epsilon_{p}-\epsilon_{d}$ and $U_{d}$ decrease.

From the calculations for the SDW wave functions, we should set $\epsilon_{p}-\epsilon_{d}$ and $U_{d}$ small so that the SDW phase does not occupy a huge region near half-filling. In Figures 15 and 16, we show energy gains for both the SDW and SC states for $U_{d}=8, t_{p p}=0,0.2$ and $\epsilon_{p}-\epsilon_{d}=2$, where the right hand side and left hand side indicate the hole-doped and electron-doped case, respectively. Solid symbols indicate the results for $8 \times 8$ and open symbols for $6 \times 6$. For this set of parameters the SDW region extends up to 20 percent doping and the pure $d$-wave phase exists outside of the SDW phase. The $d$-wave phase may be possibly identified with superconducting phase in the overdoped region in the high- $T_{c}$ superconductors.

\subsection{Stripes and Its Coexistence with Superconductivity}

Incommensurate magnetic and charge peaks have been observed from the elastic neutron-scattering experiments in the underdoped region of the Nd-doped

$\mathrm{La}_{2-x-y} \mathrm{Nd}_{y} \mathrm{Sr}_{x} \mathrm{CuO}_{4}$ [118] (Figure 17). Recent neutron experiments have also revealed the incommensurate spin structures [119-123]. Rapid decrease of the Hall resistivity in this region suggests that the electric con- 


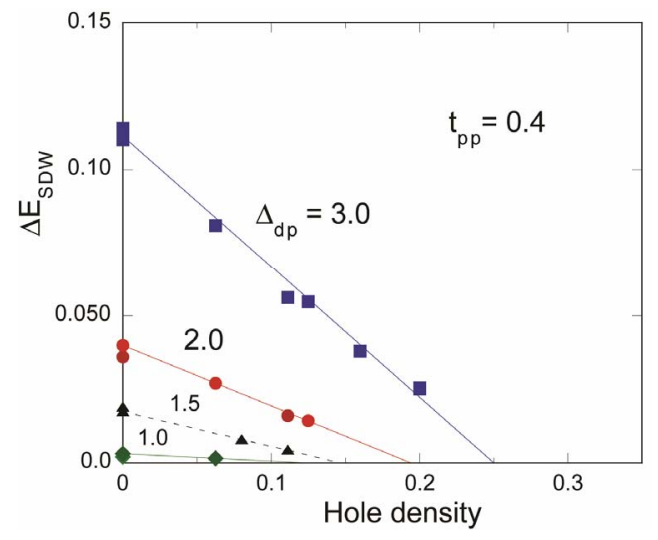

Figure 13. Energy gain per site $\left(E_{\text {normal }}-E\right) / N$ in the SDW state as a function of hole density $\delta$ for the threeband Hubbard model. Parameters are $t_{p p}=0.4$ and $U_{d}=8$ in $t_{d p}$ units. From the top, $\Delta_{d p} \equiv \epsilon_{p}-\epsilon_{d}=3,2,1.5$ and 1 . The results are for $6 \times 6,8 \times 8,10 \times 10$ and $16 \times 12$ systems. Antiperiodic and periodic boundary conditions are imposed in $x$ - and $y$-direction, respectively. Monte Carlo statistical errors are smaller than the size of symbols. Curves are a guide to the eye [24].

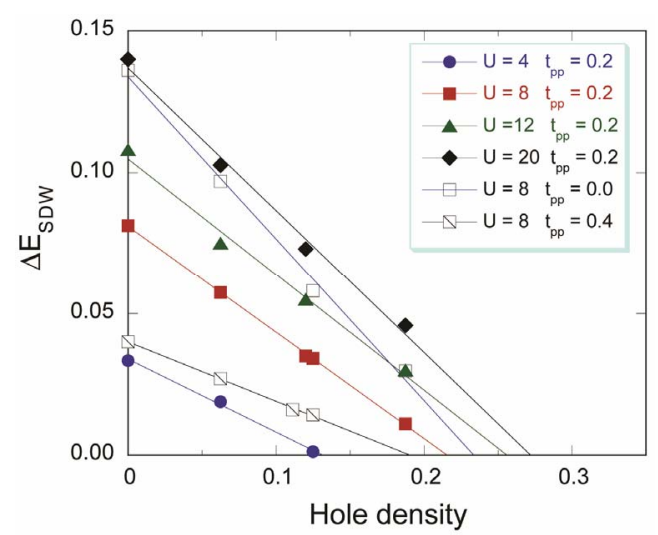

Figure 14. Uniform SDW energy gain per site with reference to the normal-state energy as a function of the hole density for the three-band Hubbard model. Data are from $8 \times 8,10 \times 10,12 \times 12$ and $16 \times 12$ systems for $\epsilon_{p}-\epsilon_{d}=2$. For solid symbols $U_{d}=4$ (circles), $U_{d}=8$ (squares), $U_{d}=12$ (triangles) and $U_{d}=20$ (diamonds) for $t_{p p}=0.2$. For open symbols $U_{d}=8$ and $t_{p p}=0$, and for open squares with slash $U_{d}=8$ and $t_{p p}=0.4$. The lines are a guide to the eye. The Monte Carlo statistical errors are smaller than the size of symbols [47].

duction is approximately one dimensional [124]. The angle-resolved photo-emission spectroscopy measurements also suggested a formation of two sets of onedimensional Fermi surface [125]. Then it has been proposed that these results might be understood in the framework of the stripe state where holes are doped in the domain wall between the undoped spin-density-wave

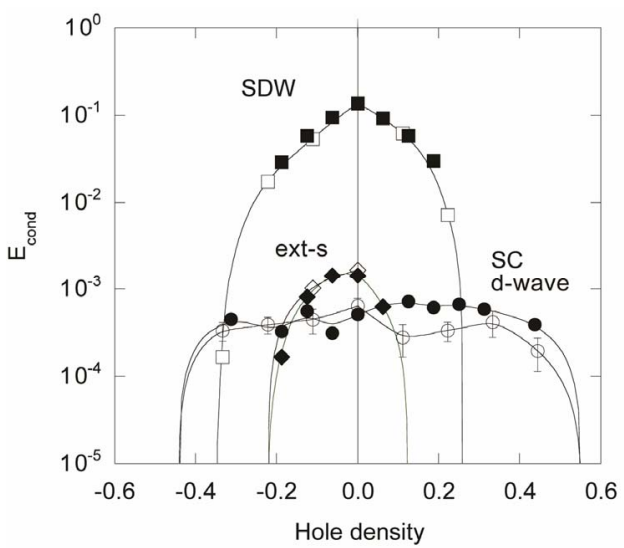

Figure 15. Condensation energy per site as a function of hole density for the three-band Hubbard model where $t_{p p}=0.0, \epsilon_{p}-\epsilon_{d}=2$ and $U_{d}=8$. Circles and squares denote the energy gain per site with reference to the normal-state energy for $d$-wave, ext-s wave and SDW states, respectively. For extremely small doping rate, the extended $s$-wave state is more favorable than the $d$-wave state. Solid symbols are for $8 \times 8$ and open symbols are for $6 \times 6$. Curves are a guide to the eye.

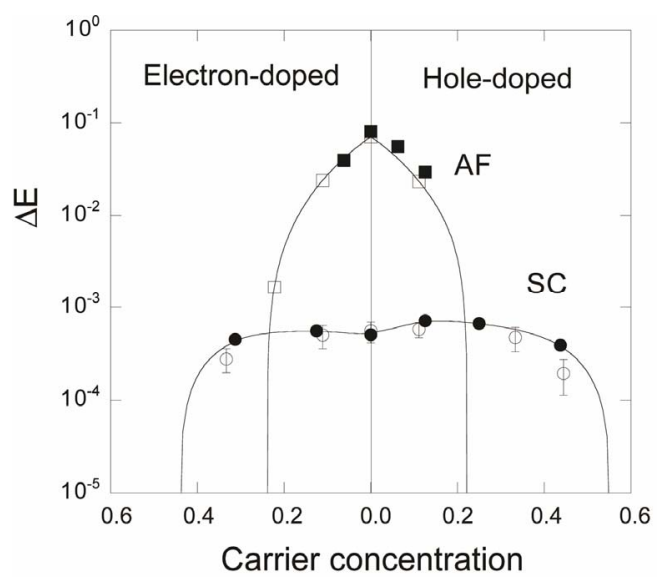

Figure 16. Condensation energy per site as a function of hole density for the three-band Hubbard model where $t_{p p}=0.2, \epsilon_{p}-\epsilon_{d}=2$ and $U_{d}=8$. Circles and squares denote the energy gain per site with reference to the normal-state energy for $d$-wave and SDW states, respectively. Solid symbols are for $8 \times 8$ and open symbols are for $6 \times 6$. Curves are a guide to the eye.

domains. This state is a kind of incommensurate SDW state. It was also shown that the incommensurability is proportional to the hole density in the low-doping region in which the hole number per stripe is half of the site number along one stripe $[118,120]$. A static magnetically ordered phase has been observed by $\mu$ SR over a wide range of $\mathrm{SC}$ phase for $0.05<x<0.1$ in $\mathrm{La}_{2-x} \mathrm{Sr}_{x} \mathrm{CuO}_{4}$ (LSCO) [126]. Thus the possibility of superconductivity that occurs in the stripe state is a subject of great interest [127-130]. The incommensurate magnetic scattering 
spots around $(\pi, \pi)$ were observed in the SC phase in the range of $0.05<x<0.13$ in the elastic and inelastic neutron-scattering experiments with LSCO [127,128,130]. The hole dependence of the incommensurability and the configuration of the spots around the Bragg spot in the SC phase indicated the vertical stripe. The neutronscattering experiments have also revealed that a diagonal spin modulation occurs across the insulating spin-glass phase in $\mathrm{La}_{2-x} \mathrm{Sr}_{x} \mathrm{CuO}_{4}$ for $0.02 \leq x \leq 0.05$, where a one-dimensional modulation is rotated by 45 degrees from the stripe in the SC phase. The incommensurability $\delta$ versus hole density is shown in Figure 18 schematically $[129,130]$. The diagonal stripe changes into the vertical stripe across the boundary between the insulating and SC phase.

Let us investigate the doped system from the point of modulated spin structures [131-141]. The stripe SDW state has been studied theoretically by using the meanfiled theory [132-136]. They found that the stripe state appears when an incommensurate nesting becomes

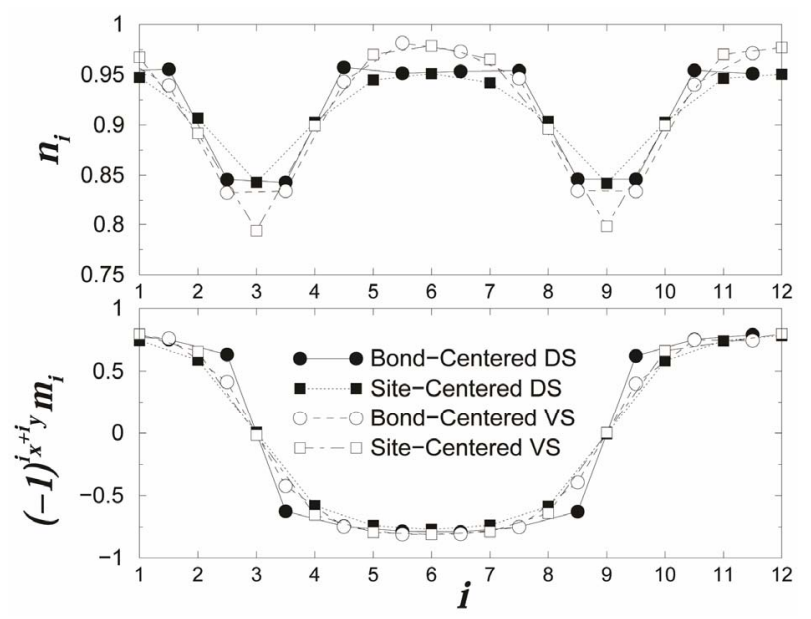

Figure 17. Charge and spin density as a function of the distance for a striped state [50].

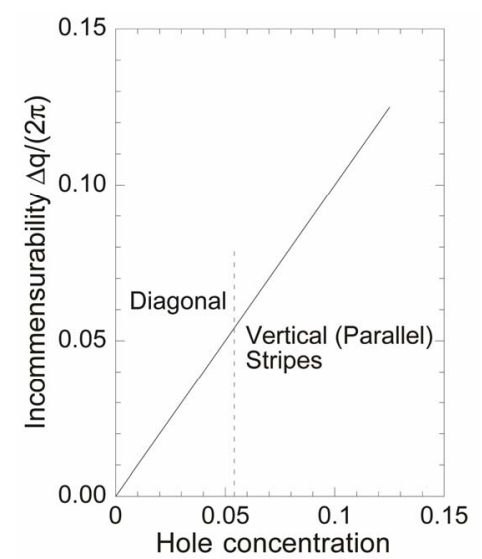

Figure 18. Schematic illustration of the incommensurability versus hole density. favorable in the hole-doped 2D Hubbard model. When the electron correlation correlation is strong or intermediate, it was shown that the stripe state is more stable than the commensurate spin-density-wave state with the wave vector $(\pi, \pi)$ in the ground state of the $2 \mathrm{D}$ Hubbard model by using the variational Monte Carlo method [131]. It has also been confirmed by the same means that the stripe states are stabilized in the $d-p$ model [48]. The purpose of this section is to examine whether the superconductivity can coexist with static stripes in the 2D Hubbard model in a wider doping region and investigate the doping dependence of the coexisting state.

We consider the 2D Hubbard model on a square lattice. We calculate the variational energy in the coexistent state that is defined by

$$
\psi_{\text {coexist }}=P_{N_{e}} P_{G} \phi_{\text {coexist }}^{M F},
$$

where $\phi_{\text {coexist }}^{M F}$ is a mean-field wave function. The effective mean-field Hamiltonian for the coexisting state is [105] represented by

$$
H_{M F}=\sum_{i j}\left(c_{i \uparrow}^{\dagger} c_{i \downarrow}\right)\left(\begin{array}{cc}
H_{i j \uparrow} & F_{i j} \\
F_{j i}^{*} & -H_{j i \downarrow}
\end{array}\right)\left(\begin{array}{c}
c_{j \uparrow} \\
c_{j \downarrow}^{\dagger}
\end{array}\right),
$$

where the diagonal terms describe the incommensurate spin-density wave state:

$$
H_{i j \sigma}=-t_{i j}-\mu+\frac{U}{2}\left[n_{i}+\operatorname{sign}(\sigma)(-1)^{x_{i}+y_{i}} m_{i}\right] \delta_{i j},
$$

where $\mu$ is the chemical potential. The vertical stripe state is represented by the charge density $n_{i}$ and the spin density $m_{i}$ that are spatially modulated as

$$
\begin{gathered}
n_{i}=1-\sum_{\ell} \alpha / \cosh \left(\frac{y_{i}-Y_{\ell}}{\xi_{c}}\right), \\
m_{i}=m \prod_{\ell} \tanh \left(\frac{y_{i}-Y_{\ell}}{\xi_{c}}\right),
\end{gathered}
$$

where $Y_{\ell}$ denotes the position of vertical stripes. The amplitude $\alpha$ is fixed by $\sum_{i} n_{i}=N_{e}$. The off-diagonal terms in Equation (38) are defined in terms of the $d$-wave SC gap as

$$
F_{i j}=\sum_{\hat{e}} \Delta_{i j} \delta_{j i+\hat{e}},
$$

where $\hat{e}= \pm \hat{x}, \pm \hat{y}$ (unit vectors). We consider two types of the spatially inhomogeneous superconductivity: anti-phase and in-phase defined as

$$
\begin{gathered}
\Delta_{i . i+\hat{x}}=\Delta \cos \left(q_{y}\left(y_{i}-Y\right)\right), \\
\Delta_{i, i+\hat{y}}=-\Delta \cos \left(q_{y}\left(y_{i}-Y+\hat{y} / 2\right)\right),
\end{gathered}
$$

and 


$$
\begin{gathered}
\Delta_{i, i+\hat{x}}=\Delta\left|\cos \left(q_{y}\left(y_{i}-Y\right)\right)\right|, \\
\Delta_{i, i+\hat{y}}=-\Delta\left|\cos \left(q_{y}\left(y_{i}-Y+\hat{y} / 2\right)\right)\right|,
\end{gathered}
$$

respectively. Here, $\boldsymbol{q}=(0,2 \pi \delta)$ and $\delta$ is a incommensurability given by the stripe's periodicity in the $y$ direction with regard to the spin. The anti-phase (inphase) means that the sign if the superconducting gap is (is not) changed between nearest domain walls.

The wave function $\psi_{\text {coexist }}^{0}$ is made from the solution of the Bogoliubov-de Gennes equation represented by

$$
\begin{gathered}
\sum_{j}\left(H_{i j \uparrow} u_{j}^{\lambda}+F_{i j} v_{j}^{\lambda}\right)=E^{\lambda} u_{i}^{\lambda}, \\
\sum_{j}\left(F_{j i}^{*} u_{j}^{\lambda}-H_{j i \downarrow} v_{j}^{\lambda}\right)=E^{\lambda} v_{i}^{\lambda} .
\end{gathered}
$$

The Bogoliubov quasiparticle operators are written in the form

$$
\begin{aligned}
& \alpha_{\lambda}=\sum_{i}\left(u_{i}^{\lambda} c_{i \uparrow}+v_{i}^{\lambda} c_{i \downarrow}^{\dagger}\right) \quad\left(E^{\lambda}>0\right), \\
& \alpha_{\bar{\lambda}}=\sum_{i}\left(u_{i}^{\bar{\lambda}} c_{i \uparrow}+v_{i}^{\bar{\lambda}} c_{i \downarrow}^{\dagger}\right) \quad\left(E^{\bar{\lambda}}<0\right) .
\end{aligned}
$$

Then the coexistence wave function is written as $[105,142]$

$$
\begin{aligned}
\psi_{\text {coexist }}^{0} & =P_{N_{e}} \prod_{\lambda} \alpha_{\lambda} \alpha_{\bar{\lambda}}^{?}|0\rangle \\
& =C P_{N_{e}} \exp \left(-\sum_{i j}\left(U^{-1} V\right)_{i j} c_{i \uparrow}^{?} c_{j \downarrow}\right)|0\rangle \\
& =C^{\prime}\left(\sum_{i j}\left(U^{-1} V\right)_{i j} c_{i \uparrow}^{?} c_{j \downarrow}\right)^{N_{e} / 2}|0\rangle,
\end{aligned}
$$

for constants $C$ and $C^{\prime}$. The calculations are performed for the wave function $\psi_{\text {coexist }}=P_{G} \psi_{\text {coexist }}^{0}$. The variational parameters are $\mu, m, g, \xi_{c}$ and $\xi_{s}$. The system parameters were chosen as $t^{\prime}=-0.20$ and $U=8$ suitable for cuprate superconductors. It has been shown that the "anti-phase" configuration is more stable than the "in-phase" one [105].

Here, the system parameters are $t^{\prime}=-0.2$ and $U=8$. We use the periodic boundary condition in the $x$-direction and anti-periodic one in the $y$-direction. In Figure 19, we show the total energy of the coexistent state, $E_{\text {coexist }}$, as a function of the SC gap $\Delta$ for the cases of anti-phase and in-phase. The SC condensation energy $\Delta E_{\text {coexist }}$ is estimated as $0.0008 t$ per site at the hole density 0.125 on the $12 \times 8$ lattice with periodic boundary condition in $x$-direction and antiperiodic one in $y$-direction. $\Delta E_{\text {coexist }}$ in the coexistence state is defined as the decrease of energy due to finite $\Delta$. If we use $t \sim 0.5 \mathrm{eV}$, this is evaluated as $\sim 0.4 \mathrm{meV}$. The SC condensation energy per site is shown as a function of hole density in Figure 20. One finds that $\Delta E_{\text {coexist }}$ in the stripe state decreases as the hole density decreases. This tendency is reasonable since the SC order is weakened in the domain of the incommensurate SDW because of the vanishingly small carrier concentration contributing the superconductivity in this domain. This behavior is consistent with the SC condensation energy estimated from the specific heat measurements [143].

There is a large renormalization of the Fermi surface due to the correlation effect in the striped state [144]. We considered the next-nearest transfer $t^{\prime}$ in the trial function as a variational parameter $\tilde{t}^{\prime}$. In Figure 21,

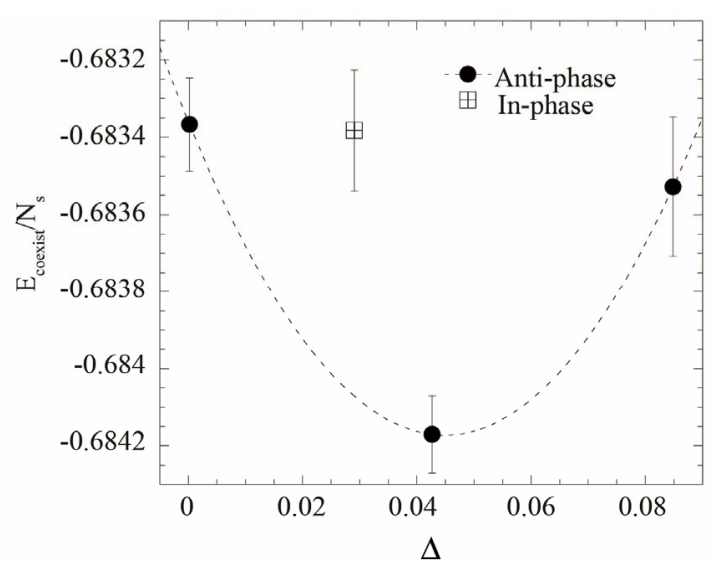

Figure 19. Coexistent state energy per site $E_{\text {coexist }} / N_{s}$ versus $\Delta$ for the case of 84 electrons on $12 \times 8$ sites with $U=8$ and $t^{\prime}=-0.2$. Here the vertical stripe state has 8-lattice periodicity for the hole density $p=0.125$. Only $E_{\text {coexist }} / N_{s}$ for the optimized gap is plotted for the in-phase superconductivity.

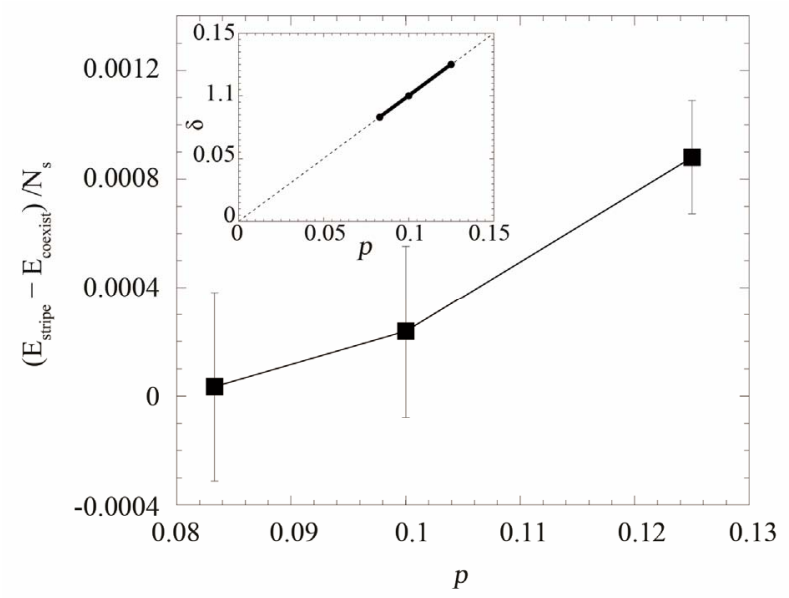

Figure 20. Superconducting condensation energy per site in the coexistence state as a function of the hole density $p=0.0833,0.10$ and 0.125 . The model is the single-band Hubbard Hamiltonian with $t^{\prime}=-\mathbf{0 . 2 0}$. The stripe interval is preserved constant. The inset shows the hole dependence of the incommensurability in the coexistent state [105]. 


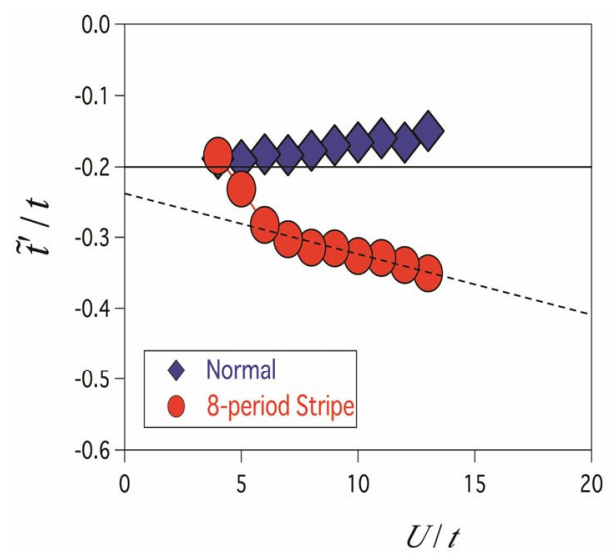

Figure 21. Optimized effective second neighbor transfer energy $\tilde{t}^{\prime} / t$ as a function of $U / t$. The system is a $16 \times 16$ lattice with $t^{\prime} / t=-0.2$ and the electron density 0.875 .

optimized values of $\tilde{t}^{\prime} / t$ for the striped state are shown as a function of $U / t$. The $\tilde{t}^{\prime} / t$ increases as $U / t$ increases. We also mention that the optimized $t^{\prime \prime} / t$ almost vanishes. The renormalized Fermi surface of $\tilde{t}^{\prime} / t=-0.30,-0.40$ and -0.50 are plotted in Figure 22. The system is a $16 \times 16$ lattice with $t^{\prime} / t=-0.2$ and the electron density 0.875 . As $U / t$ is incresed, the Fermi surface is more deformed. We show the the gradient of the momentum distribution function, $\left|\nabla n_{k}\right|$, calculated in the optimized stripe state in Figure 23. The brighter areas coincide with the renormalized Fermi surface with $\tilde{t}^{\prime} / t=-0.31$ and $\tilde{t}^{\prime \prime} / t=-0.0$ for $U / t=8$.

The calculations for the three-band Hubbard model has also been done taking into account the coexistence of stripes and SC $[15,106]$. The energy of antiferromagnetic state would be lowered further if we consider the incommensurate spin correlation in the wave function. The phase diagram in Figure $\mathbf{2 4}$ presents the region of stable AF phase in the plane of $t_{p p}$ and $\Delta_{d p}=\epsilon_{p}-\epsilon_{d}$. For large $\Delta_{d p}=\epsilon_{p}-\epsilon_{d}$, we have the region of the AF state with an eight-lattice periodicity in accordance with the results of neutron-scattering measurements [118,123]. The energy at $x=1 / 16$ is shown in Figure 25 where the 4-lattice stripe state has higher energy than that for 8-lattice stripe for all the values of $t_{p p}$.

The Bogoliubov-de Gennes equation is extended to the case of three orbitals $d, p_{x}$ and $p_{y} .\left(H_{i j \sigma}\right)$ and $\left(F_{i j}\right)$ are now $3 N \times 3 N$ matrices. The energy of the state with double order parameters $\Delta$ and $m$ is shown in Figure 26 on the $16 \times 4$ lattice at the doping rate $1 / 8$. The SC condensation energy per site is evaluated as $\sim 0.00016 t_{d p}$ for $U_{d}=8, t_{p p}=0.4$ and $\epsilon_{p}-\epsilon_{d}=2$. If we use $t_{d p} \sim 1.5 \mathrm{eV}$ [89-91], we obtain $\Delta E_{\text {coexist }} \sim$ $0.24 \mathrm{meV}$ which is slightly smaller than and close to the value obtained for the single-band Hubbard model. We show the size dependence of the SC condensation

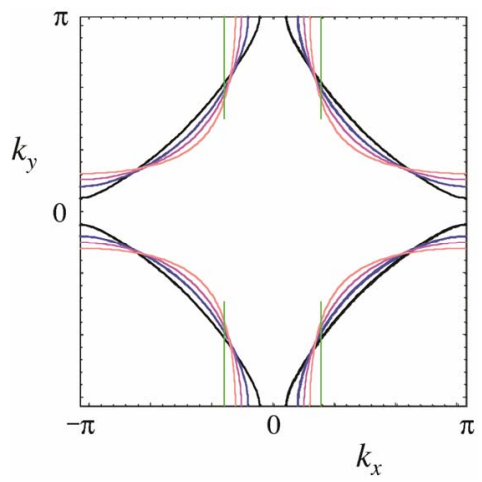

Figure 22. Renormalized quasi-Fermi surface for $\tilde{t}^{\prime} / t=-0.3,-0.4$ and -0.5 . The system is the same as that in Figure 21.

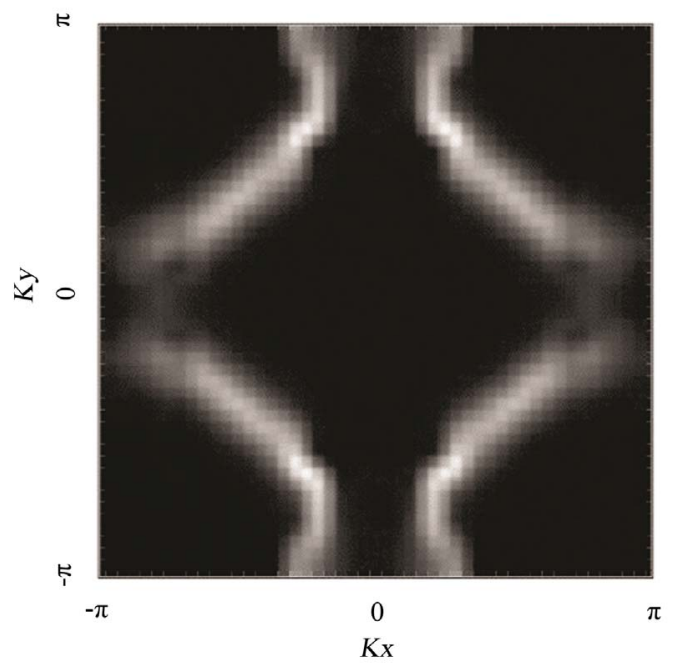

Figure 23. Contour plot of $\left|\nabla n_{k}\right|$ measured for the projected stripe state on $24 \times 24$ lattice with $t^{\prime} / t=-0.2$. The electron density is $\mathbf{0 . 8 7 5}$.

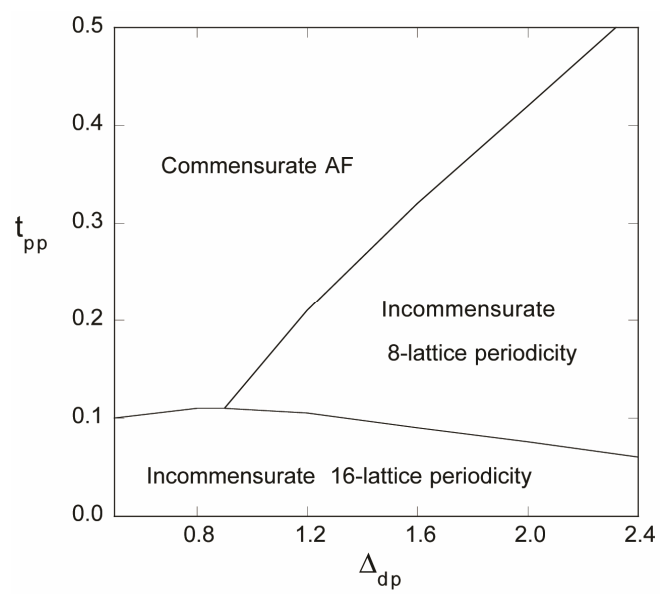

Figure 24. Phase diagram of stable antiferromagnetic state in the plane of $\Delta_{d p}=\epsilon_{p}-\epsilon_{d}$ and $t_{p p}$ obtained for $16 \times 4$ lattice. 


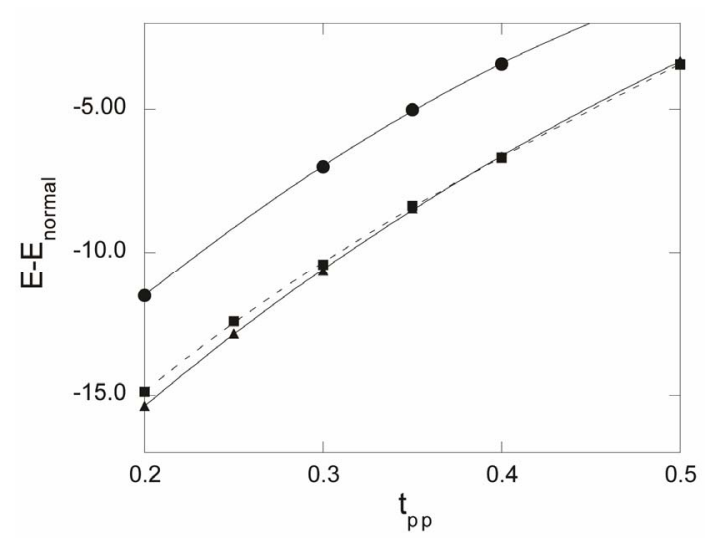

Figure 25. Energy as a function of $t_{p p}$ for $16 \times 16$ square lattice at $x=1 / 16$. Circles, triangles and squares denote the energy for 4-lattice stripes, 8-lattice stripes, and commensurate SDW, respectively, where $n$-lattice stripe is the incommensurate state with one stripe per $n$ ladders. The boundary conditions are antiperiodic in $x$-direction and periodic in $y$-direction $[47,106]$.

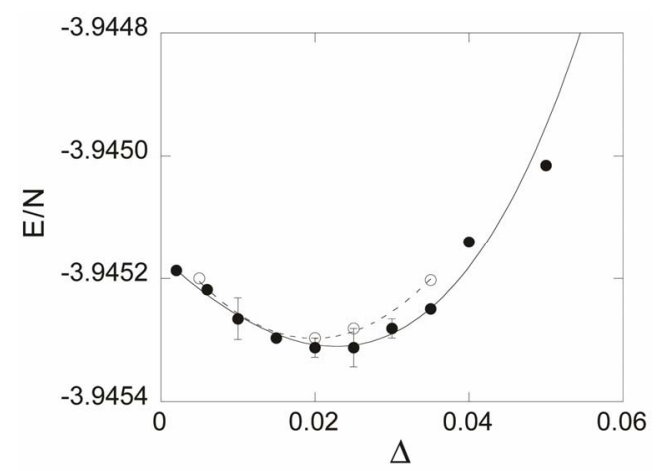

Figure 26. Energy of the coexistent state as a function of the SC order parameter for $x=0.125$ on $16 \times 4$ lattice. We assume the incommensurate antiferromagnetic order (stripe). Parameters are $\epsilon_{p}=0, \epsilon_{d}=-2, t_{p p}=0.4$ and $U_{d}=8$ in $t_{d p}$ units. For solid circles the SC gap function is taken as $\Delta_{i, i+\hat{x}}=\Delta \cos \left(Q_{x}\left(x_{i}+\hat{x} / 2\right)\right)$ and

$\Delta_{i, i+\hat{y}}=-\Delta \cos \left(Q_{x}\left(x_{i}\right)\right)$, while for the open circles

$\Delta_{i, i+\hat{x}}=\Delta \cos \left|\left(Q_{x}\left(x_{i}+\hat{x} / 2\right)\right)\right|$ and $\Delta_{i, i+\hat{y}}=-\Delta\left|\cos \left(Q_{x}\left(x_{i}\right)\right)\right|$.

$Q_{x}=2 \pi \delta=\pi / 4$.

energy for $x=0.2,0.1250 .08333$ and 0.0625 in Figure 27. We set the parameters as $\epsilon_{p}-\epsilon_{d}=2$ and $t_{p p}=0.4$ in $t_{d p}$ units, which is reasonable from the viewpoint of the density of states and is remarkably in accordance with cluster estimations [89-91], and also in the region of eight-lattice periodicity at $x=1 / 8$. We have carried out the Monte Carlo calculations up to $16 \times 16$ sites $(768$ atoms in total). In the overdoped region in the range of $0.18<x<0.28$, we have the uniform $d$-wave pairing state as the ground state. The periodicity of spatial variation increases as the doping rate $x$ decreases proportional to $1 / x$. In the figure we have the 12-lattice periodicity at $x=0.08333$ and 16-lattice periodicity at $x=0.0625$. For $x=0.2,0.125$ and 0.08333 , the results strongly suggest a finite condensation energy in the bulk limit. The SC condensation energy obtained on the basis of specific heat measurements agrees well with the variational Monte Carlo computations [99]. In general, the Monte Carlo statistical errors are much larger than those for the single-band Hubbard model. The large number of Monte Carlo steps (more than $5.0 \times 10^{7}$ ) is required to get convergent expectation values for each set of parameters.

In Figure 28 the order parameters $\Delta_{A F}$ and $\Delta_{S C}$

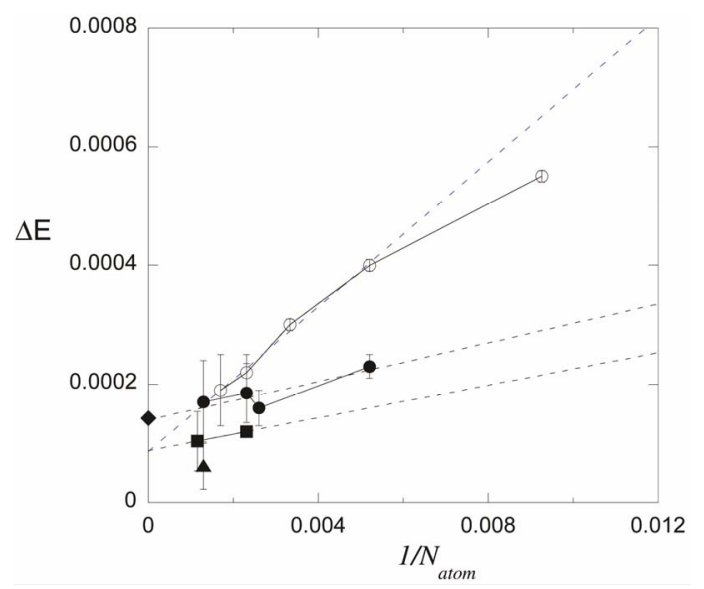

Figure 27. Energy gain due to the SC order parameter as a function of the system size $N_{\text {atom }}=3 N_{s}$. Parameters are $\epsilon_{p}=0, \epsilon_{d}=-2, t_{p p}=0.4$ and $U_{d}=8$. The open circle is for the simple $d$-wave pairing at the hole density $x=0.2$. The solid symbols indicate the energy gain of the coexistent state; the solid circle is at $x=0.125$, the solid square is at $x=0.08333$ and the solid triangle is at $x=0.0625$. The diamond shows the SC condensation energy obtained on the basis of specific heat measurements [99].

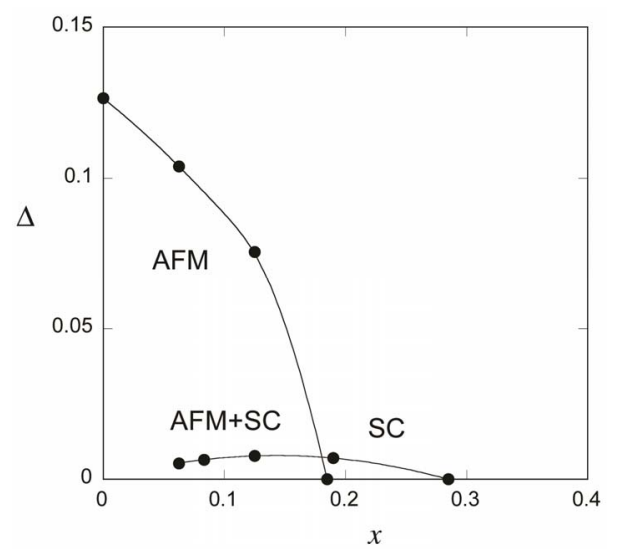

Figure 28. Phase diagram of the $d-p$ model based on the Gutzwiller wave function [106]. 
were evaluated using the formula $E_{\text {cond }}=(1 / 2) N(0) \Delta^{2}$ where $N(0)$ is the density of states. Here we have set $N(0) \sim 5 / t_{d p}$ since $N(0)$ is estimated as $N(0) \sim 2$ to $3(\mathrm{eV})^{-1}$ for optimally doped YBCO using $N(0)\left(k_{B} T_{c}\right)^{2} / 2$ [100]. The phase diagram is consistent with the recently reported phase diagram for layered cuprates [145].

\subsection{Diagonal Stripe States in the Light-Doping Region}

Here we examine whether the relationship $\delta \sim x$ holds in the lower doping region or not, and whether the diagonal stripe state is obtained in the further lower doping region [50]. The elastic neutron scattering experiments of LSCO in the light-doping region, $0.03<x<0.07$, revealed that the position of incommensurate magnetic peaks changed from $(1 / 2,1 / 2 \pm \delta)$ to $\left(1 / 2 \pm \delta^{\prime}, 1 / 2 \pm \delta^{\prime}\right)$ as $x$ becomes less than 0.06 $[129,130]$. This means that the stripe direction rotates by 45 degrees to become diagonal at this transition. In the diagonal stripe states, the magnetic peaks are observed to keep a relation $\delta \sim x$ that holds in the vertical stripe state in the low doping region.

In Figure 29, we show the incommensurability of the most stable stripe state as a function of $x$. Open squares and triangles are values for diagonal and vertical incommensurate SDW's obtained in the elastic neutron scattering experiments on LSCO, respectively. Solid squares and triangles show our results for the diagonal and vertical stripes, respectively. These results are in a good agreement with experimental data. We also found that the phase boundary $x_{\text {critical }}$ between the diagonal and vertical stripe states lies at or above 0.0625 in the

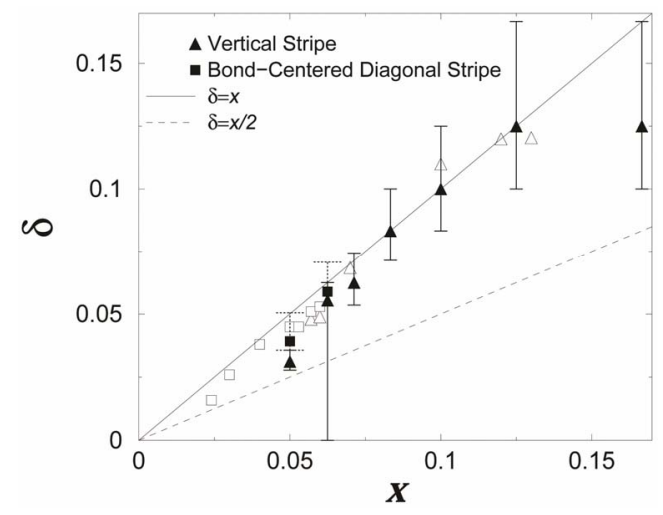

Figure 29. Incommensurability $\delta$ as a function of the hole density $x$ for $U=8$ and $t^{\prime}=-0.2$ [50]. The numerical results for the vertical and the bond-centered diagonal stripe state are represented by solid triangles and square symbols, respectively. Open triangles and squares show the results of the vertical and diagonal incommensurate SDW order observed from neutron scattering measurements, respectively [131]. case of $U=8$ and $t^{\prime}=-0.2$. The following factors may give rise to slight changes of the phase boundary $x_{\text {critical }}$ : the diagonal stripe state may be stabilized in the low-temperature-orthorhombic (LTO) phase in LSCO. The diagonal stripe state is probably stabilized further by forming a line along larger next-nearest hopping direction due to the anisotropic $t^{\prime}$ generated by the $\mathrm{Cu}-\mathrm{O}$ buckling in the LTO phase.

\subsection{Checkerboard States}

A checkerboard-like density modulation with a roughly $4 a \times 4 a$ period ( $a$ is a lattice constant) has also been observed by scanning tunneling microscopy (STM) experiments in $\mathrm{Bi}_{2} \mathrm{Sr}_{2} \mathrm{CaCu}_{2} \mathrm{O}_{8+\delta}$, [146] $\mathrm{Bi}_{2} \mathrm{Sr}_{2-x} \mathrm{La}_{x} \mathrm{CuO}_{6+\delta}$, [147] and $\mathrm{Ca}_{2-x} \mathrm{Na}_{x} \mathrm{CuO}_{2} \mathrm{Cl}_{2}$ (Na-CCOC) [148]. It is important to clarify whether these inhomogeneous states can be understood within the framework of strongly correlated electrons.

Possible several electronic checkerboard states have been proposed theoretically $[134,149,150]$. The charge density $\rho_{i}$ and spin density $m_{i}$ are spatially modulated as

$$
\begin{gathered}
\rho_{i}=\sum_{\ell} \rho_{\ell} \cos \left(Q_{\ell}^{c} \cdot\left(\boldsymbol{r}_{i}-\boldsymbol{r}_{0}\right)\right), \\
m_{i}=\sum_{\ell} m_{\ell} \cos \left(Q_{\ell}^{s} \cdot\left(\boldsymbol{r}_{i}-\boldsymbol{r}_{0}\right)\right) .
\end{gathered}
$$

where $\rho_{\ell}$ and $m_{\ell}$ are variational parameters. The striped incommensurate spin-density wave state (ISDW) is defined by a single $Q$ vector. On the other hand, the checkerboard ISDW state is described by the double-Q set; for example, vertical wave vectors

$Q_{1}^{s}=(\pi, \pi \pm 2 \pi \delta)$ and $Q_{2}^{s}=(\pi \pm 2 \pi \delta, \pi)$ describe a spin vertical checkerboard state, where two diagonal domain walls are orthogonal. Diagonal wave vectors $Q_{1}^{s}=(\pi \pm 2 \pi \delta, \pi \pm 2 \pi \delta)$ and $Q_{2}^{s}=(\pi \pm 2 \pi \delta, \pi \mp 2 \pi \delta)$ lead to a spin diagonal checkerboard state with a $1 / \delta$-period. The hole density forms the charge vertical checkerboard pattern with vertical wave vectors $Q_{1}^{c}=(0, \pm 4 \pi \delta)$ and $Q_{2}^{c}=(2 \pi \pm 4 \pi \delta, 2 \pi)$ in which the hole density is maximal on the crossing point of magnetic domain walls in the spin diagonal checkerboard state. If $\delta=1 / 8$ is assumed, the charge modulation pattern is consistent with the $4 a \times 4 a$ charge structure observed in STM experiments.

We found that the coexistent state of bond-centered four-period diagonal and vertical spin-checkerboard structure characterized by a multi-Q set is stabilized and composed of $4 \times 4$ period checkerboard spin modulation. [151] In Figure 30(a), we show the condensation energies of some heterogeneous states, $\left(E_{\text {normal }}-E_{\text {hetero }}\right) / N_{\text {site }}$, with fixing the transfer energies $t^{\prime}=-0.32$ and $t^{\prime \prime}=0.22$ suitable for $\mathrm{Bi}-2212$. The system is a $16 \times 16$ lattice with the electron-filling 


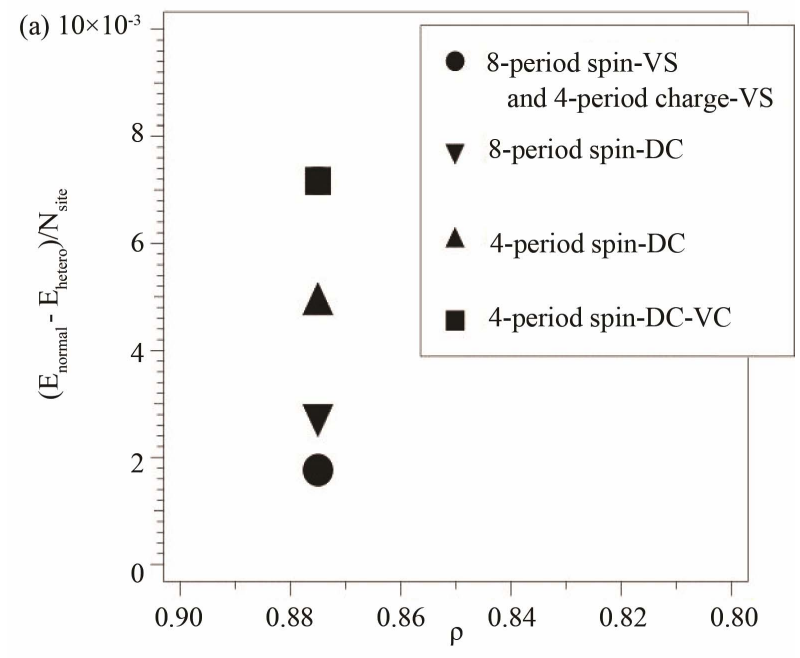

(b)

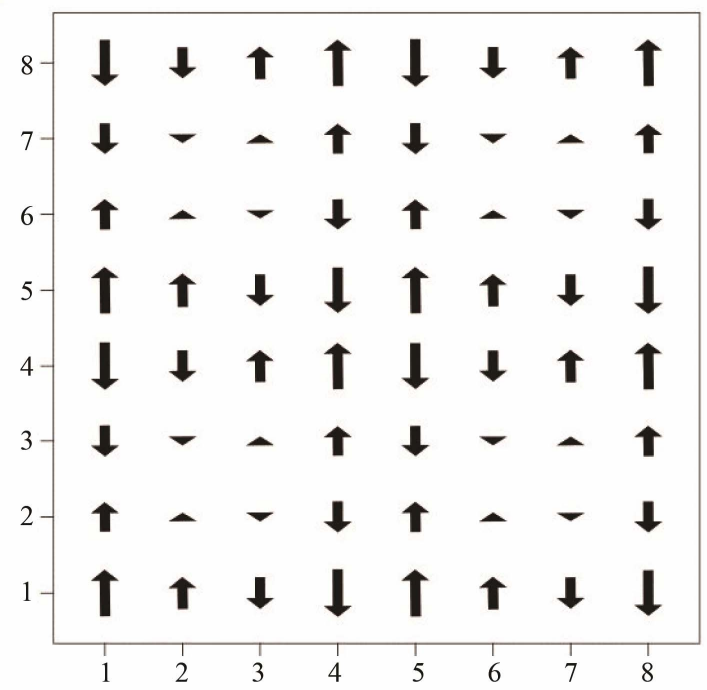

Figure 30. (a) Condensation energies of inhomogeneous states with the bond-centered magnetic domain wall. The system is a $16 \times 16$ lattice with $t^{\prime}=-0.32, t^{\prime \prime}=0.22$, and $U=8$ for the case of $\rho=0.875$. The static error bars are smaller than the size of symbols; (b) Expectation value of the spin density $m_{i}$ measured in the four-period spin-DCVC solution. The length of arrows is proportional to the spin density.

$\rho=N_{e} / N_{\text {site }}=0.875$. The energy of the normal state $E_{\text {normal }}$ is calculated by adopting $m_{\ell}=\rho_{\ell}=0$. In our calculations, the condensation energies of both bondcentered stripe and checkerboard states are always larger than those of site-centered stripe and checkerboard states. The vertical stripe state is not suitable in this parameter set since this state is only stabilized with the LSCO-type band. The four-period spin-diagonal checkerboard (DC) state is significantly more stable than the eight-period spin-DC state. We found that the coexistent state of the bond-centered four-period spin-DC and four-period spin-vertical checkerboard (VC) with $\rho_{\ell}=0$ is most stable, as shown in Figure 30(a). The measured expectation value of the spin density is shown in Figure 30(b).

\subsection{Improved Gutzwiller Function}

We have presented the results based on the Gutzwiller functions for the normal state, SDW state and BCS state. We must consider a method to go beyond the Gutzwiller function-based Monte Carlo method. One method to achieve this purpose is to multiply the Jastrow correlation operators to take into account the intersite correlations. The simplest possible candidate is an introduction of the diagonal intersite correlation factor [152]:

$$
\psi_{\text {Jastrow }}=\prod_{j \ell(\neq 0)} \prod_{\sigma \sigma^{\prime}}\left[1-(1-g(\ell)) n_{j \sigma} n_{j+\ell \sigma^{\prime}}\right] \psi_{n},
$$

for the variational parameter $g(\ell)$. We have investigated the 2D Hubbard model by using the JastrowGutzwiller function [111]. The ground-state energy is lowered considerably by considering the intersite correlations such as nearest neighbor and next nearest neighbor spin and charge correlations.

Here we consider the method to improve the wave functions by an off-diagonal Jastrow correlation operators $[94,95,153]$. The off-diagonal correlation factors are more effective to lower the ground state energy in 2D systems. Let us consider the wave functions $\psi^{(m)}$ defined in the following way: [95]

$$
\begin{gathered}
\psi^{(1)}=\psi_{G}=\mathrm{e}^{-\alpha V} \psi_{0}, \\
\psi^{(2)}=\mathrm{e}^{-\lambda K} \mathrm{e}^{-\alpha V} \psi_{0}, \\
\psi^{(3)}=\mathrm{e}^{-\lambda^{\prime} K} \mathrm{e}^{-\alpha^{\prime} V} \psi^{(2)}, \\
\ldots \quad \ldots
\end{gathered}
$$

and so on, where $K$ denotes the kinetic part of the Hamiltonian

$$
K=-t \sum_{\langle i j\rangle \sigma}\left(c_{i \sigma}^{\dagger} c_{j \sigma}+\text { h.c. }\right)
$$

and $V$ denotes the on-site Coulomb interaction. $\lambda$, $\lambda^{\prime}, \lambda^{\prime \prime}, \alpha, \alpha^{\prime}$ and $\alpha^{\prime \prime}$ are variational parameters. It is considered that $\psi^{(m)}$ approaches the true ground state wave function as $m$ grows larger since the ground state wave function is written as

$$
\psi=\mathrm{e}^{-\beta H} \psi_{0} \simeq \mathrm{e}^{-\epsilon_{1} K} \mathrm{e}^{-\epsilon_{1} V} \cdots \mathrm{e}^{-\epsilon_{m} K} \mathrm{e}^{-\epsilon_{m} V} \psi_{0},
$$

for large $\beta=\epsilon_{1}+\cdots+\epsilon_{m}$ and small $\epsilon_{i}(i=1, \cdots, m)$. If we can extrapolate the expectation values from the data for $\psi^{(1)}, \psi^{(2)}, \cdots$, we can evaluate correct expectation values.

The computations are performed by using the discrete Hubbard-Stratonovich transformation as described in 
Section 3.1. In the evaluation of the expectation values we generate the Monte Carlo samples by the importance sampling [95] with the weight function $|w|=\left|w_{\uparrow} w_{\downarrow}\right|$ where

$$
w_{\sigma}=\operatorname{det}\left(\phi_{0}^{\sigma \dagger} \exp \left(V^{\sigma}(u, \alpha)\right) \cdots \exp \left(V^{\sigma}(s, \alpha) \phi_{0}^{\sigma}\right)\right) \text {. }
$$

Since the Monte Carlo samplings are generated with the weight $|w|$, the expectation values are calculated with the sign of $w$ in the summation over the generated samples. In our calculations the negative sign problem has become less serious due to the variational treatment, although we encounter the inevitable negative sign problem in the standard projector Monte Carlo approaches [154].

In Figure 31 the energy is shown as a function of $m$ where the SDW and normal states are chosen as the initial state $\psi_{0}$. The extrapolated values for different initial states coincide with each other within Monte Carlo statistical errors. The energy expectation values as a function of $U$ for $8 \times 8$ square lattice are presented in Figure 32 for $\psi_{n}=\psi^{(1)}, \psi_{A F}, \psi^{(3)}$. The extrapolated curve is shown by the solid curve and the results obtained by the quantum Monte Carlo simulation (QMC) [28] are also shown as a reference. A good agreement between two calculations support the method although the QMC gives slightly higher energy for $U=8$.

One can formulate an approach to consider the BCS function with correlation operators. [96] For this end the electron-hole transformation is introduced for the down spin and the up-spin electrons are unaltered [155]. We show the energy versus $1 / m$ in Figure 33 for $\psi^{(m)}$ and $\psi_{s}^{(m)}$. From an extrapolation to the limit $m \rightarrow \infty$, both formulations predict the same limiting value for the energy. The energy is lowered considerably due to the

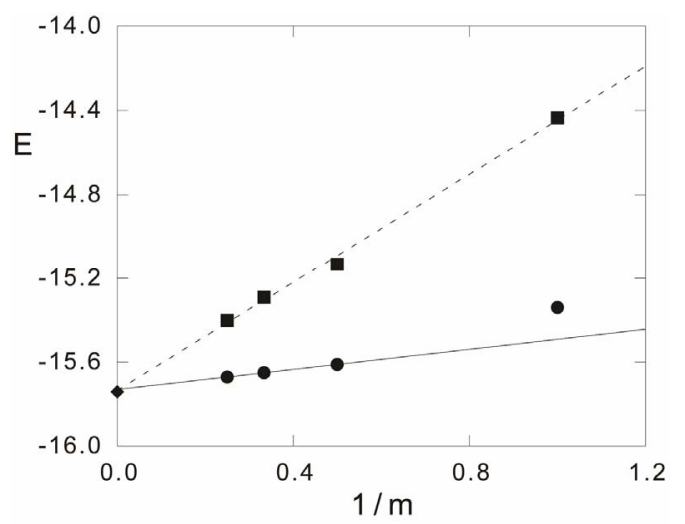

Figure 31. Energy as a function of $1 / m$ for the single-band Hubbard model for $N_{e}=14$ and $U=4$ on the lattice of $4 \times 4$ sites. For the upper and lower curves, the initial wave function $\psi_{0}$ is the Fermi sea and SDW state, respectively. The diamond indicates the exact value obtained from the diagonalization [95].

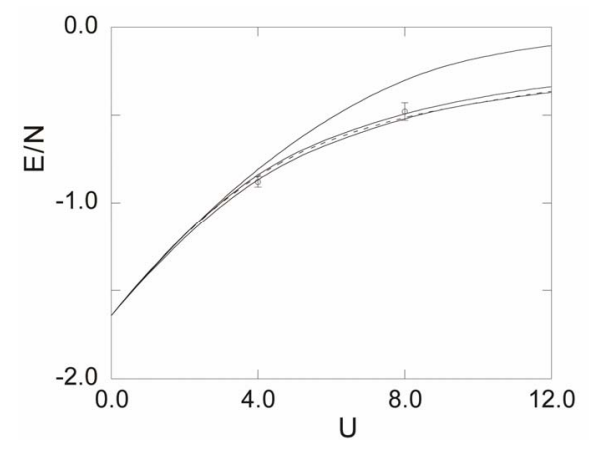

Figure 32. Energy as a function of $U$ for $8 \times 8$ lattice at half-filling for the single-band Hubbard model. From the top the energies for $\psi_{n}, \psi_{A F}, \psi^{(3)}$ and extrapolated values are shown. The quantum Monte Carlo results are shown by open circles [95].

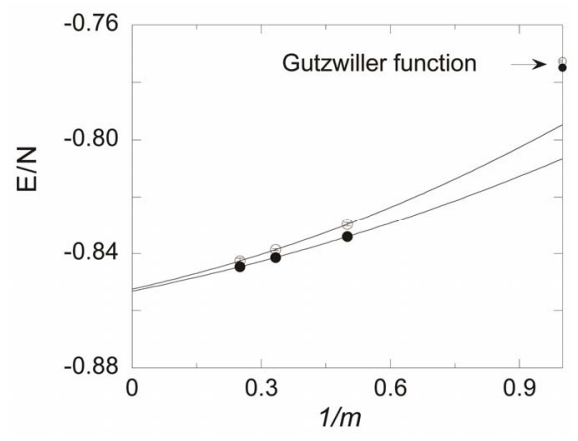

Figure 33. The energy versus $1 / m$ for the single-band Hubbard model on the lattice of $10 \times 10$ sites. Solid and open symbols are obtained for $\psi_{m}$ with the normal and $d$-wave state initial functions $\psi_{0}$ and $\psi_{B C S}$, respectively. Parameters are given by $U=8, t^{\prime}=-0.09$ and $N_{e}=80$ [96].

correlation operators compared to that for the Gutzwiller function. The energy in the $d$-wave SC state is always lower than that in the normal state for each $m$. The energy gain in the SC state remains the same order after the multiplication of correlation factors.

\subsection{1. $T_{c}$ and $\epsilon_{p}-\epsilon_{d}$}

Relationships between $T_{c}$ and structural features in cuprate high-temperature superconductors are very interesting. Torrance and Metzger found the first such relationship between $T_{c}$ and the Madelung potential difference $\Delta V_{M}$ [156]. Here $\Delta V_{M}$ is the potential difference between $\mathrm{Cu}$ and $\mathrm{O}$ sites in the $\mathrm{CuO}_{2}$ plane. $T_{c}$ was found to increase with decreasing $\Delta V_{M}$. There is an interesting tendency of increasing $T_{c}$ with increasing relative ratio of hole density at oxygen site against that at copper site [117].

Here we show the results obtained by using the perturbation theory [62-66], There have been many 
similar works by making some kind of approximation such as random phase approximation (RPA) [157-159], fluctuation-exchange approximation (FLEX) [160-163], effective spin-fluctuation method [4,164,165], and perturbation theory in terms of $U$ [166-168]. An application was made for $\mathrm{Sr}_{2} \mathrm{RuO}_{4}$ where we need to consider th emulti-band structure $\alpha$ and $\beta$ orbitals [169], and also to the three-dimensional d-p model [170]. In our formulation the gap function is written as

$$
\Delta=\exp \left(-\frac{2}{x U_{d}^{2}}\right) \text {. }
$$

The exponent $x$ indicates the strength of superconductivity. The results are in Figures 34 and 35 [171]. As shown in the figure, for positive $\epsilon_{p}-\epsilon_{d}$, with increase of $\epsilon_{p}-\epsilon_{d}$ the exponent $x$ increases monotonously. This means the increase of superconducting gap and so of $T_{c}$, and is consistent with the wide-range tendency of the variational Monte Carlo calculation [24,172]. This tendency can be understood in terms of

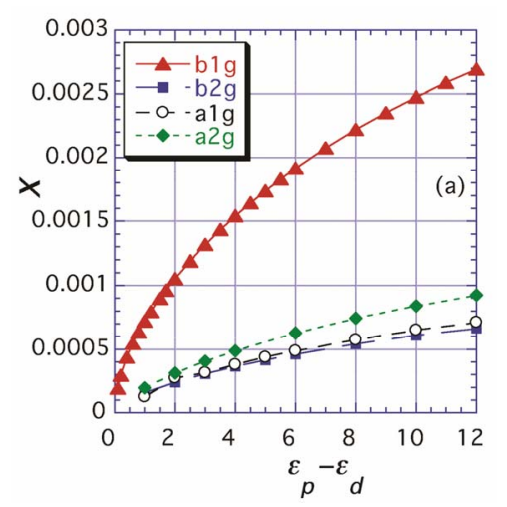

Figure 34. The exponent $x$ (superconductivity strength) as a function of $\epsilon_{p}-\epsilon_{d}$, where the level difference $\epsilon_{p}-\epsilon_{d}$ is positive.

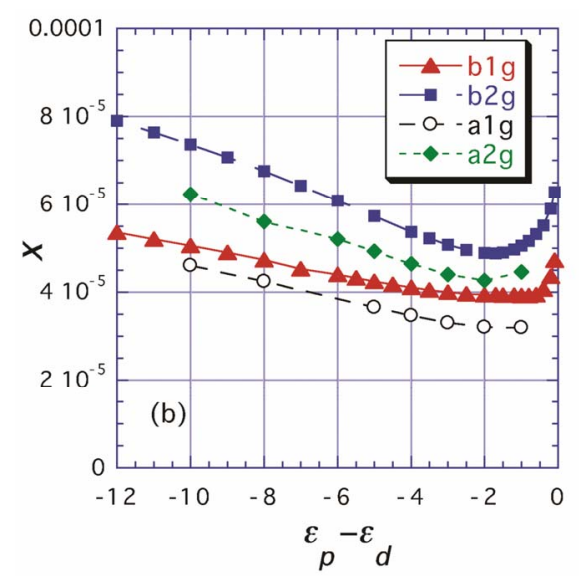

Figure 35. The exponent $x$ as a function of $\epsilon_{p}-\epsilon_{d}$, where the level difference $\epsilon_{p}-\epsilon_{d}$ is negative.

$$
U_{\text {eff }}=U_{d}\left\langle u_{\boldsymbol{k}}^{0}\right\rangle^{2}=\frac{U_{d}}{4}\left(1+\frac{1}{\sqrt{1+16 t_{d p}^{2} /\left(\epsilon_{p}-\epsilon_{d}\right)^{2}}}\right)^{2},
$$

where $u_{k}^{0}$ is the weight ofd electrons. This clearly indicates that increase of $\epsilon_{p}-\epsilon_{d}$ leads to the increase of $U_{\text {eff }}$ and subsequently of $x$. In the case of $\epsilon_{p}-\epsilon_{d}<0$, we take account of finite Coulomb repulsion $U_{p}$ on oxygen sites. The effective interaction coming from $U_{p}$ is similarly given by the susceptibility with the weight of $p$ electrons. The results of $x$ with $U_{p} / U_{d}=6 / 8$ indicates that all four types of even parity $\left(b_{1 g}, b_{2 g}\right.$, $a_{1 g}$ and $a_{2 g}$ ) SC strength values increase, so that $T_{c}$ is raised, as the absolute value $\left|\epsilon_{p}-\epsilon_{d}\right|$ increases in this region. This result shows that $U_{p}$ also plays an important role as well.

Let us give a discussion on this result. Increase of $\left|\epsilon_{p}-\epsilon_{d}\right|$ in the region of $\epsilon_{p}-\epsilon_{d}<0$ means decrease of $\Delta V_{M}=|e|\left(V_{M}^{\mathrm{O}}-V_{M}^{\mathrm{Cu}}\right)$ since $\epsilon_{p}-\epsilon_{d}=\left(A_{2}^{\mathrm{O}}-I_{3}^{\mathrm{Cu}}\right)+|e|\left(V_{M}^{\mathrm{O}}-V_{M}^{\mathrm{Cu}}\right)$, where $A_{2}^{\mathrm{O}}$ is the second electron affinity of oxygen atom and $I_{3}^{C u}$ is the third electron ionicity of copper atom and $e$ is the charge of electron. Therefore, this relation is consistent with the systematics reported in [156]. With increase of the distance of the apex oxygen away from the $\mathrm{CuO}_{2}$ plane, cuprate superconductors are known to increase $T_{c}$ [173]. The accompanying raise of $\epsilon_{d}$ should tend to increase $T_{c}$.

The Coulomb interaction between $\mathrm{p}$ electrons on oxygen atom will raise the level of $\mathrm{p}$ electrons effectively. This leads to the lowering of $\mathrm{p}$ hole level $\epsilon_{p}$ or the raise of $\epsilon_{d}$ relatively. This indicates that $T_{c}$ will be increased by the Coulomb interaction between $\mathrm{p}$ electrons.

\section{Quantum Monte Carlo Studies}

\subsection{Quantum Monte Carlo Method}

The Quantum Monte Carlo (QMC) method is a numerical method that is employed to simulate the behavior of correlated electron systems. We outline the QMC method in this section. The Hamiltonian is the Hubbard model that contains the on-site Coulomb repulsion and is written as

$$
H=-\sum_{i j \sigma} t_{i j}\left(c_{i \sigma}^{\dagger} c_{j \sigma}+\text { h.c. }\right)+U \sum_{j} n_{j \uparrow} n_{j \downarrow},
$$

where $c_{j \sigma}^{\dagger}\left(c_{j \sigma}\right)$ is the creation (annihilation) operator of an electron with spin $\sigma$ at the $j$-th site and $n_{j \sigma}=c_{j \sigma}^{\dagger} c_{j \sigma} \cdot t_{i j}$ is the transfer energy between the sites $i$ and $j . t_{i j}=t$ for the nearest-neighbor bonds. For all other cases $t_{i j}=0 . U$ is the on-site Coulomb energy. 
The number of sites is $N$ and the linear dimension of the system is denoted as $L$. The energy unit is given by $t$ and the number of electrons is denoted as $N_{e}$.

In a Quantum Monte Carlo simulation, the ground state wave function is

$$
\psi=\mathrm{e}^{-\tau H} \psi_{0},
$$

where $\psi_{0}$ is the initial one-particle state represented by a Slater determinant. For large $\tau, \mathrm{e}^{-\tau H}$ will project out the ground state from $\psi_{0}$. We write the Hamiltonian as $H=K+V$ where $K$ and $V$ are the kinetic and interaction terms of the Hamiltonian in Equation (64), respectively. The wave function in Equation (65) is written as

$$
\psi=\left(\mathrm{e}^{-\Delta \tau(K+V)}\right)^{M} \psi_{0} \approx\left(\mathrm{e}^{-\Delta \tau K} \mathrm{e}^{-\Delta \tau V}\right)^{M} \psi_{0},
$$

for $\tau=\Delta \tau \cdot M$. Using the Hubbard-Stratonovich transformation $[27,94]$, we have

$$
\begin{aligned}
& \exp \left(-\Delta \tau U n_{i \uparrow} n_{i \downarrow}\right) \\
& =\frac{1}{2} \sum_{s_{i}= \pm 1} \exp \left(2 a s_{i}\left(n_{i \uparrow}-n_{i \downarrow}\right)-\frac{1}{2} U \Delta \tau\left(n_{i \uparrow}+n_{i \downarrow}\right)\right),
\end{aligned}
$$

for $(\tanh a)^{2}=\tanh (\Delta \tau U / 4)$ or $\cosh (2 a)=\mathrm{e}^{\Delta \tau U / 2}$. The wave function is expressed as a summation of the one-particle Slater determinants over all the configurations of the auxiliary fields $s_{j}= \pm 1$. The exponential operator is expressed as

$$
\begin{aligned}
\left(\mathrm{e}^{-\Delta \tau K} \mathrm{e}^{-\Delta \tau V}\right)^{M}= & \frac{1}{2^{N M}} \sum_{\left\{s_{i}(\ell)\right\}} \prod_{\sigma} B_{M}^{\sigma}\left(s_{i}(M)\right) \\
& \times B_{M-1}^{\sigma}\left(s_{i}(M-1)\right) \cdots B_{1}^{\sigma}\left(s_{i}(1)\right),
\end{aligned}
$$

where we have defined

$$
B_{\ell}^{\sigma}\left(\left\{s_{i}(\ell)\right\}\right)=\mathrm{e}^{-\Delta \tau K_{\sigma}} \mathrm{e}^{-V_{\sigma}\left(\left\{s_{i}(\ell)\right\}\right)},
$$

for

$$
\begin{gathered}
V_{\sigma}\left(\left\{s_{i}\right\}\right)=2 a \sigma \sum_{i} s_{i} n_{i \sigma}-\frac{1}{2} U \Delta \tau \sum_{i} n_{i \sigma}, \\
K_{\sigma}=-\sum_{i j} t_{i j}\left(c_{i \sigma}^{\dagger} c_{j \sigma}+\text { h.c. }\right) .
\end{gathered}
$$

The ground-state wave function is

$$
\psi=\sum_{m} c_{m} \phi_{m}
$$

where $\phi_{m}$ is a Slater determinant corresponding to a configuration $m=\left\{s_{i}(\ell)\right\} \quad(i=1, \cdots, N ; \ell=1, \cdots, M)$ of the auxiliary fields:

$$
\phi_{m}=\prod_{\sigma} B_{M}^{\sigma}\left(s_{i}(M)\right) \cdots B_{1}^{\sigma}\left(s_{i}(1)\right) \psi_{0} \equiv \phi_{m}^{\uparrow} \phi_{m}^{\downarrow} .
$$

The coefficients $c_{m}$ are constant real numbers: $c_{1}=c_{2}=\cdots$. The initial state $\psi_{0}$ is a one-particle state. If electrons occupy the wave numbers $k_{1}, k_{2}, \cdots, k_{N_{\sigma}}$ for each spin $\sigma, \psi_{0}$ is given by the product $\psi_{0}^{\uparrow} \psi_{0}^{\downarrow}$ where $\psi_{0}^{\sigma}$ is the matrix represented as [31]

$$
\left(\begin{array}{cccc}
\mathrm{e}^{i k_{1} \cdot \eta_{1}} & \mathrm{e}^{i k_{2} \cdot \eta_{1}} & \cdots & \mathrm{e}^{i k_{N_{\sigma}} \cdot \eta_{1}} \\
\mathrm{e}^{i k_{1} \cdot r_{2}} & \mathrm{e}^{i k_{2} \cdot r_{2}} & \cdots & \mathrm{e}^{i k_{N_{\sigma}} \cdot r_{2}} \\
\vdots & \vdots & \ddots & \vdots \\
\mathrm{e}^{i k_{1} \cdot r_{N}} & \mathrm{e}^{i k_{2} \cdot r_{N}} & \cdots & \mathrm{e}^{i k_{N_{\sigma}} \cdot r_{N}}
\end{array}\right) .
$$

$N_{\sigma}$ is the number of electrons for spin $\sigma$. In actual calculations we can use a real representation where the matrix elements are $\cos \left(k_{i} \cdot r_{j}\right)$ or $\sin \left(k_{i} \cdot r_{j}\right)$. In the real-space representation, the matrix of $V_{\sigma}\left(\left\{s_{i}\right\}\right)$ is a diagonal matrix given as

$$
V_{\sigma}\left(\left\{s_{i}\right\}\right)=\operatorname{diag}\left(2 a \sigma s_{1}-U \Delta \tau / 2, \cdots, 2 a \sigma s_{N}-U \Delta \tau / 2\right) .
$$

The matrix elements of $K_{\sigma}$ are

$$
\left(K_{\sigma}\right)_{i j}=\left\{\begin{array}{lc}
-t & i, j \text { are nearest neighbors } \\
0 & \text { otherwise. }
\end{array}\right.
$$

$\phi_{m}^{\sigma}$ is an $N \times N_{\sigma}$ matrix given by the product of the matrices $\mathrm{e}^{-\Delta \tau K_{\sigma}}, \mathrm{e}^{V_{\sigma}}$ and $\psi_{0}^{\sigma}$. The inner product is thereby calculated as a determinant [38],

$$
\left\langle\phi_{m}^{\sigma} \phi_{n}^{\sigma}\right\rangle=\operatorname{det}\left(\phi_{m}^{\sigma \dagger} \phi_{n}^{\sigma}\right) \text {. }
$$

The expectation value of the quantity $Q$ is evaluated as

$$
\langle Q\rangle=\frac{\sum_{m n}\left\langle\phi_{m} Q \phi_{n}\right\rangle}{\sum_{m n}\left\langle\phi_{m} \phi_{n}\right\rangle} .
$$

If $Q$ is a bilinear operator $Q_{\sigma}$ for spin $\sigma$, we have $\left\langle Q_{\sigma}\right\rangle$

$$
\begin{aligned}
& =\frac{\sum_{m n}\left\langle\phi_{m}^{\sigma} Q_{\sigma} \phi_{n}^{\sigma}\right\rangle\left\langle\phi_{m}^{-\sigma} \phi_{n}^{-\sigma}\right\rangle}{\sum_{m n}\left\langle\phi_{m}^{\sigma} \phi_{n}^{\sigma}\right\rangle\left\langle\phi_{m}^{-\sigma} \phi_{n}^{-\sigma}\right\rangle}=\frac{\sum_{m n}\left\langle\phi_{m}^{\sigma} Q_{\sigma} \phi_{n}^{\sigma}\right\rangle \operatorname{det}\left(\phi_{m}^{-\sigma \dagger} \phi_{n}^{-\sigma}\right)}{\sum_{m n} \operatorname{det}\left(\phi_{m}^{\sigma \dagger} \phi_{n}^{\sigma}\right) \operatorname{det}\left(\phi_{m}^{-\sigma \dagger} \phi_{n}^{-\sigma}\right)} \\
= & \sum_{m n} \frac{\operatorname{det}\left(\phi_{m}^{\sigma \dagger} \phi_{n}^{\sigma}\right) \operatorname{det}\left(\phi_{m}^{-\sigma \dagger} \phi_{n}^{-\sigma}\right)}{\sum_{m^{\prime} n^{\prime}} \operatorname{det}\left(\phi_{m^{\prime}}^{\sigma \dagger} \phi_{n^{\prime}}^{\sigma}\right) \operatorname{det}\left(\phi_{m^{\prime}}^{-\sigma^{\dagger}} \phi_{n^{\prime}}^{-\sigma}\right)} \times \frac{\left\langle\phi_{m}^{\sigma} Q_{\sigma} \phi_{n}^{\sigma}\right\rangle}{\left\langle\phi_{m}^{\sigma} \phi_{n}^{\sigma}\right\rangle} .
\end{aligned}
$$

The expectation value with respect to the Slater determinants $\left\langle\phi_{m}^{\sigma} Q_{\sigma} \phi_{n}^{\sigma}\right\rangle$ is evaluated using the singleparticle Green's function $[31,38]$,

$$
\frac{\left\langle\phi_{m}^{\sigma} c_{i \sigma} c_{j \sigma}^{\dagger} \phi_{n}^{\sigma}\right\rangle}{\left\langle\phi_{m}^{\sigma} \phi_{n}^{\sigma}\right\rangle}=\delta_{i j}-\left(\phi_{n}^{\sigma}\left(\phi_{m}^{\sigma \dagger} \phi_{n}^{\sigma}\right)^{-1} \phi_{m}^{\sigma \dagger}\right)_{i j} .
$$

In the above expression, 


$$
P_{m n} \equiv \operatorname{det}\left(\phi_{m}^{\sigma} \phi_{n}^{\sigma}\right) \operatorname{det}\left(\phi_{m}^{-\sigma} \phi_{n}^{-\sigma}\right)
$$

can be regarded as the weighting factor to obtain the Monte Carlo samples. Since this quantity is not necessarily positive definite, the weighting factor should be $\left|P_{m n}\right|$; the resulting relationship is,

$$
\begin{aligned}
\left\langle Q_{\sigma}\right\rangle & =\sum_{m n} P_{m n}\left\langle Q_{\sigma}\right\rangle_{m n} / \sum_{m n} P_{m n} \\
& =\sum_{m n}\left|P_{m n}\right| \operatorname{sign}\left(P_{m n}\right)\left\langle Q_{\sigma}\right\rangle_{m n} / \sum_{m n}\left|P_{m n}\right| \operatorname{sign}\left(P_{m n}\right)
\end{aligned}
$$

where $\operatorname{sign}(a)=a /|a|$ and

$$
\left\langle Q_{\sigma}\right\rangle_{m n}=\frac{\left\langle\phi_{m}^{\sigma} Q_{\sigma} \phi_{n}^{\sigma}\right\rangle}{\left\langle\phi_{m}^{\sigma} \phi_{n}^{\sigma}\right\rangle} .
$$

This relation can be evaluated using a Monte Carlo procedure if an appropriate algorithm, such as the Metropolis or heat bath method, is employed [94]. The summation can be evaluated using appropriately defined Monte Carlo samples,

$$
\left\langle Q_{\sigma}\right\rangle=\frac{\frac{1}{n_{M C}} \sum_{m n} \operatorname{sign}\left(P_{m n}\right)\left\langle Q_{\sigma}\right\rangle_{m n}}{\frac{1}{n_{M C}} \sum_{m n} \operatorname{sign}\left(P_{m n}\right)},
$$

where $n_{M C}$ is the number of samples. The sign problem is an issue if the summation of $\operatorname{sign}\left(P_{m n}\right)$ vanishes within statistical errors. In this case it is indeed impossible to obtain definite expectation values.

\subsection{Quantum Monte Carlo Diagonalization}

\subsubsection{Basic Method and Optimization}

Quantum Monte Carlo diagonalization (QMD) is a method for the evaluation of $\left\langle Q_{\sigma}\right\rangle$ without the negative sign problem [41]. A bosonic version of this method was developed before in Ref.[174]. The configuration space of the probability $\left\|P_{m n}\right\|$ in Equation (84) is generally very strongly peaked. The sign problem lies in the distribution of $P_{m n}$ in the configuration space. It is important to note that the distribution of the basis functions $\phi_{m} \quad(m=1,2, \cdots)$ is uniform since $c_{m}$ are constant numbers: $c_{1}=c_{2}=\cdots$. In the subspace $\left\{\phi_{m}\right\}$, selected from all configurations of auxiliary fields, the right-hand side of Equation (78) can be determined. However, the large number of basis states required to obtain accurate expectation values is beyond the current storage capacity of computers. Thus we use the variational principle to obtain the expectation values.

From the variational principle,

$$
\langle Q\rangle=\frac{\sum_{m n} c_{m} c_{n}\left\langle\phi_{m} Q \phi_{n}\right\rangle}{\sum_{m n} c_{m} c_{n}\left\langle\phi_{m} \phi_{n}\right\rangle},
$$

where $c_{m}(m=1,2, \cdots)$ are variational parameters. In order to minimize the energy

$$
E=\frac{\sum_{m n} c_{m} c_{n}\left\langle\phi_{m} H \phi_{n}\right\rangle}{\sum_{m n} c_{m} c_{n}\left\langle\phi_{m} \phi_{n}\right\rangle},
$$

the equation $\partial E / \partial c_{n}=0 \quad(n=1,2, \cdots)$ is solved for,

$$
\sum_{m} c_{m}\left\langle\phi_{n} H \phi_{m}\right\rangle-E \sum_{m} c_{m}\left\langle\phi_{n} \phi_{m}\right\rangle=0 .
$$

If we set

$$
\begin{gathered}
H_{m n}=\left\langle\phi_{m} H \phi_{n}\right\rangle, \\
A_{m n}=\left\langle\phi_{m} \phi_{n}\right\rangle,
\end{gathered}
$$

the eigen-equation is

$$
H u=E A u,
$$

for $u=\left(c_{1}, c_{2}, \cdots\right)^{t}$. Since $\phi_{m} \quad(m=1,2, \cdots)$ are not necessarily orthogonal, $A$ is not a diagonal matrix. We diagonalize the Hamiltonian $A^{-1} H$, and then calculate the expectation values of correlation functions with the ground state eigenvector.

In Quantum Monte Carlo simulations an extrapolation is performed to obtain the expectation values for the ground-state wave function. If $M$ is large enough, the wave function in Equation (72) will approach the exact ground-state wave function, $\psi_{\text {exact }}$, as the number of basis functions, $N_{\text {states }}$, is increased. If the number of basis functions is large enough, the wave function will approach, $\psi_{\text {exact }}$, as $M$ is increased. In either case the method employed for the reliable extrapolation of the wave function is a key issue in calculating the expectation values. The variance method was recently proposed in variational and Quantum Monte Carlo simulations, where the extrapolation is performed as a function of the energy variance. We can expect linearity in some cases [175]:

$$
\langle Q\rangle-Q_{\text {exact }} \propto v,
$$

where $v$ denotes the variance defined as

$$
v=\frac{\left\langle(H-\langle H\rangle)^{2}\right\rangle}{\langle H\rangle^{2}}
$$

and $Q_{\text {exact }}$ is the expected exact value of the quantity $Q$.

The simplest procedure for optimizing the groundstate wave function is to increase the number of basis states $\left\{\phi_{m}\right\}$ by random sampling. First, we set $\tau$ and $M$, for example, $\tau=0.1,0.2, \cdots$, and $M=20,30, \cdots$. We denote the number of basis functions as $N_{\text {states }}$. We start with $N_{\text {states }}=100 \sim 300$ and then increase up to 10,000 . This procedure can be outlined as follows: 
1) Generate the auxiliary fields $s_{i} \quad(i=1, \cdots, N)$ in $B_{\ell}^{\sigma}\left(\left\{s_{i}\right\}\right)$ randomly for $\ell=1, \cdots, M$ for $\phi_{m}$ $\left(m=1, \cdots, N_{\text {states }}\right)$, and generate $N_{\text {states }}$ basis wave function $\left\{\phi_{m}\right\}$.

2) Evaluate the matrices $H_{m n}=\left\langle\phi_{m} H \phi_{n}\right\rangle$ and $A_{m n}=\left\langle\phi_{m} \phi_{n}\right\rangle$, and diagonalize the matrix $A^{-1} H$ to obtain $\psi=\sum_{m} c_{m} \phi_{m}$. Then calculate the expectation values and the energy variance.

3) Repeat the procedure from 1) after increasing the number of basis functions.

For small systems this random method produces reliable energy results. The diagonalization plays an importance producing fast convergence. In order to lower the ground-state energy efficiently, we can employ a genetic algorithm [176] to generate the basis set from the initial basis set. One idea is to replace some parts of $\left\{s_{i}(\ell)\right\} \quad(i=1, \cdots, N ; \ell=1, \cdots, M)$ in $\phi_{n}$ that has the large weight $\left|c_{n}\right|^{2}$ to generate a new basis function $\phi_{n}^{\prime}$. The new basis function $\phi_{n}^{\prime}$ obtained in this way is expected to also have a large weight and contribute to $\psi$. The details of the method are shown in Ref.[41].

\subsubsection{Ground State Energy and Correlation} Functions

The energy as a function of the variance is presented in Figures 36-38 for $4 \times 4,6 \times 2$ and $6 \times 6$, respectively. To obtain these results the genetic algorithm was employed to produce the basis functions except the open symbols in Figure 4. The $4 \times 4$ where $N_{e}=10$ in Figure 2 is the energy for the closed shell case up to 2000 basis states. The other two figures are for open shell cases, where evaluations were performed up to 3000 states. We show the results for the $4 \times 4,6 \times 2$ and $6 \times 6$ systems in Table 2 .

The Figure 39 is the momentum distribution function $n(\boldsymbol{k})$,

$$
n(\boldsymbol{k})=\frac{1}{2} \sum_{\sigma}\left\langle c_{\boldsymbol{k} \sigma}^{\dagger} c_{\boldsymbol{k} \sigma}\right\rangle,
$$

for $14 \times 14$ sites where the results for the Gutzwiller VMC and the QMD are indicated. The Gutzwiller function gives the results that $n(k)$ increases as $k$ approaches $k_{F}$ from above the Fermi surface. This is clearly unphysical. This flaw of the Gutzwiller function near the Fermi surface is not observed for the QMD result.

\subsubsection{Spin Gap in the Hubbard Ladder}

Here we show the results for one-dimensional models. The ground state of the 1D Hubbard model is no longer Fermi liquid for $U>0$. The ground state is insulating at half-filling and metallic for less than half-filling. The Figure 40 is the spin and charge correlation functions, $S(k)$ and $C(k)$, as a function of the wave number, for
Table 2. Ground state energy per site from the Hubbard model. The boundary conditions are periodic in both directions. The current results are presented under the column labeled QMD. The constrained path Monte Carlo (CPMC) results are from Ref.[38]. The column VMC is the results obtained for the optimized variational wave function $\psi_{\lambda}^{(2)}$ except for $6 \times 2$ for which $\psi_{\lambda}^{(1)}$ is employed. The QMC results are from Ref.[35]. Exact results are obtained using diagonalization [177].

\begin{tabular}{|c|c|c|c|c|c|c|c|}
\hline Size & $N_{e}$ & $U$ & QMD & VMC & CPMC & QMC & Exact \\
\hline $4 \times 4$ & 10 & 4 & -1.2237 & $-1.221(1)$ & -1.2238 & & -1.2238 \\
\hline $4 \times 4$ & 14 & 4 & -0.9836 & $-0.977(1)$ & -0.9831 & & -0.9840 \\
\hline $4 \times 4$ & 14 & 8 & $-0.732(2)$ & $-0.727(1)$ & -0.7281 & & -0.7418 \\
\hline $4 \times 4$ & 14 & 10 & $-0.656(2)$ & $-0.650(1)$ & & & -0.6754 \\
\hline $4 \times 4$ & 14 & 12 & $-0.610(4)$ & $-0.607(2)$ & -0.606 & & -0.6282 \\
\hline $6 \times 2$ & 10 & 2 & $-1.058(1)$ & $-1.040(1)$ & & & -1.05807 \\
\hline $6 \times 2$ & 10 & 4 & $-0.873(1)$ & $-0.846(1)$ & & & -0.8767 \\
\hline $6 \times 6$ & 34 & 4 & $-0.921(1)$ & $-0.910(2)$ & & -0.925 & \\
\hline $6 \times 6$ & 36 & 4 & $-0.859(2)$ & $-0.844(2)$ & & -0.8608 & \\
\hline
\end{tabular}

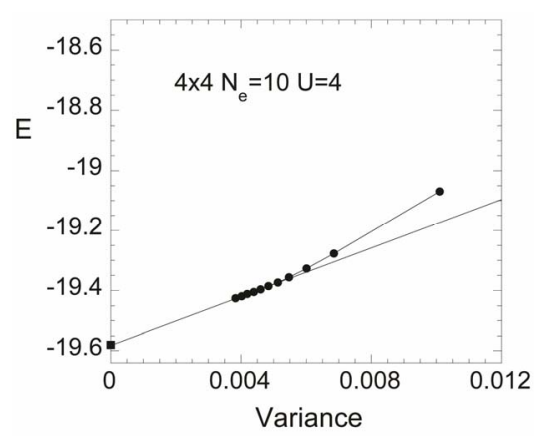

Figure 36. Energy as a function of the variance for $4 \times 4$, $U=4$ and $N_{e}=10$. The square is the exact result. The data fit using a straight line using the least-square method as the variance is reduced. We started with $N_{\text {states }}=100$ (first solid circle) and then increase up to 2000.

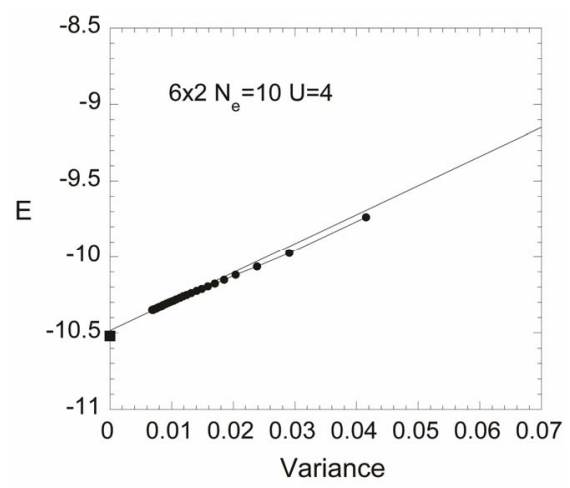

Figure 37. Energy as a function of the variance for $6 \times 2$ $N_{e}=10$ and $U=4$. The square is the exact value obtained using exact diagonalization. 


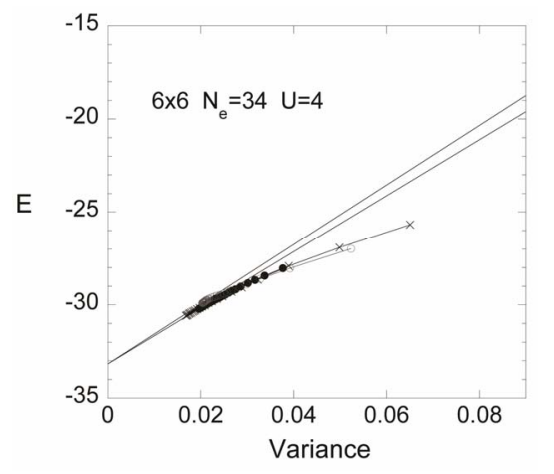

Figure 38. Energy as a function of the variance $v$ for $6 \times 6$. with the periodic boundary conditions. Solid circles and crosses are data obtained from the QMD method for two different initial configurations of the auxiliary fields. Gray open circles show results obtained from the $1 / 2^{N}$-renormalization method with 300 basis wave functions.

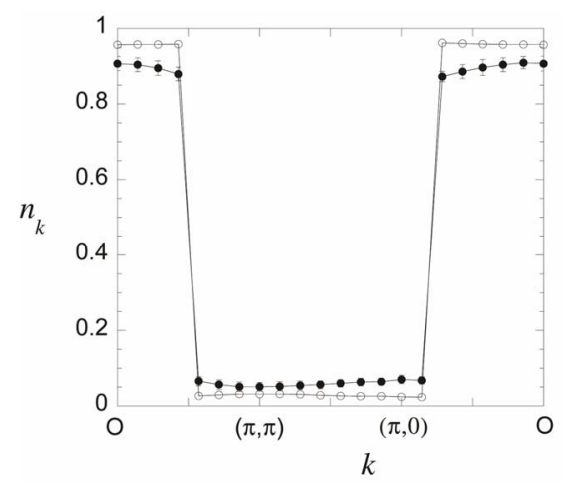

Figure 39. Momentum distribution function for the $14 \times 14$ lattice. Parameters are $U=4$ and $N_{e}=146$. The boundary conditions are periodic in both directions. The results for the Gutzwiller function (open circle) are also provided.

the 1D Hubbard model where $N=80$. The $2 k_{F}$ singularity can be clearly identified where the dotted line is for $U=0$. The spin correlation is enhanced and the charge correlation function is suppressed slightly because of the Coulomb interaction.

The spin correlation function $S(\boldsymbol{k})$ for the Hubbard ladder is presented in Figure 41, where $U=4$ and $t_{d}=1 . S(\boldsymbol{k})$ is defined as

$$
S(\boldsymbol{k})=\frac{1}{N} \sum_{i \ell, j \ell^{\prime}} \mathrm{e}^{i k \cdot\left(\boldsymbol{R}_{i \ell}-\boldsymbol{R}_{j \ell^{\prime}}\right)}\left\langle\left(n_{\ell i \uparrow}-n_{\ell i \downarrow}\right)\left(n_{\ell^{\prime} j \uparrow}-n_{\ell^{\prime} j \downarrow}\right)\right\rangle,
$$

where $\boldsymbol{R}_{i \ell}$ denotes the site $(i, \ell) \quad(\ell=1,2)$. We use the convention that $\boldsymbol{k}=\left(k, k_{y}\right)$ where $k_{y}=0$ and $\pi$ indicate the lower band and upper band, respectively. There are four singularities at $2 k_{F 1}, 2 k_{F 2}, k_{F 1}-k_{F 2}$, and $k_{F 1}+k_{F 2}$ for the Hubbard ladder, where $k_{F 1}$ and $k_{F 2}$ are the Fermi wave numbers of the lower and upper band, respectively.

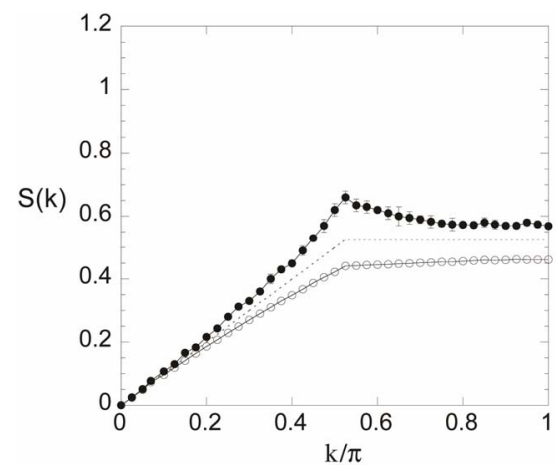

Figure 40. Spin (solid circle) and charge (open circle) correlation functions obtained from the QMD method for the one-dimensional Hubbard model with 80 sites. The number of electrons is 66 . We set $U=4$ and use the periodic boundary condition.

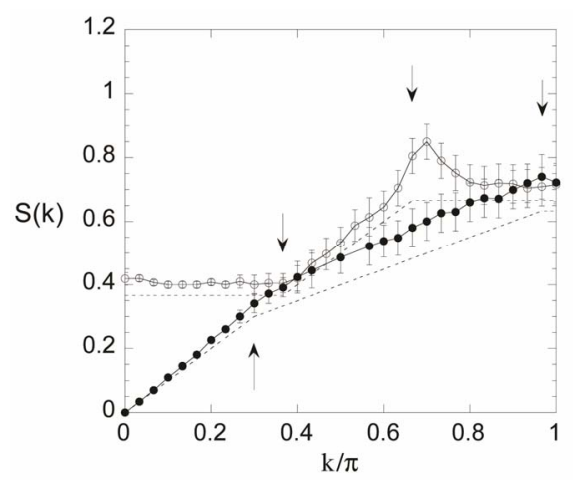

Figure 41. Spin correlation function obtained from the QMD method for the ladder Hubbard model for $60 \times 2$ sites with periodic boundary condition. The number of electrons is 80 and $U=4$. The upper line is for the upper band and the lower line is for the lower band. Singularities are at $k_{F 1}-k_{F 2}, 2 k_{F 2}, k_{F 1}+k_{F 2}$ and $2 k_{F 1}$ from left. The dotted lines are for $U=0$.

It has been expected that the charge gap opens up as $U$ turns on at half-filling for the Hubbard ladder model. In Figure 42 the charge gap at half-filling is shown as a function of $U$. The charge gap is defined as

$$
\Delta_{c}=E\left(N_{e}+2\right)+E\left(N_{e}-2\right)-2 E\left(N_{e}\right),
$$

where $E\left(N_{e}\right)$ is the ground state energy for the $N_{e}$ electrons. The charge gap in Figure 42 was estimated using the extrapolation to the infinite system from the data for the $20 \times 2,30 \times 2$, and $40 \times 2$ systems. The data suggest the exponentially small charge gap for small $U$ or the existence of the critical value $U_{c}$ in the range of $0 \leq U_{c}<1.5$, below which the charge gap vanishes.

\subsubsection{Magnetization in 2D Hubbard Model}

The ground state of the 2D Hubbard model at half-filling is antiferromagnetic for $U>0$ because of the nesting due to the commensurate vector $Q=(\pi, \pi)$. The Gutz- 
willer function predicts that the magnetization

$$
m=\left|\frac{1}{N} \sum_{j}\left(n_{j \uparrow}-n_{j \downarrow}\right) \mathrm{e}^{i Q \cdot R_{j}}\right|
$$

increases rapidly as $U$ increases and approaches $m=1$ for large $U$. In Figure 43, the QMD results are presented for $m$ as a function of $U$. The previous results obtained using the QMC method are plotted as open circles. The gray circles are for the $\lambda$-function VMC method and squares are the Gutzwiller VMC data. Clearly, the magnetization is reduced considerably because of the fluctuations, and is smaller than the Gutzwiller VMC method by about 50 percent.

\subsection{Pair Correlation Function}

The pair correlation function $D_{\alpha \beta}$ is defined by

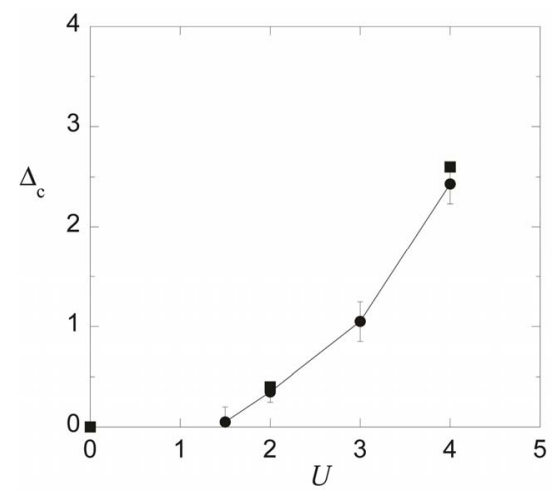

Figure 42. Charge gap as a function of $U$ for $t_{d}=1$ (circles). The DMRG results (squares) are provided for comparison [58].

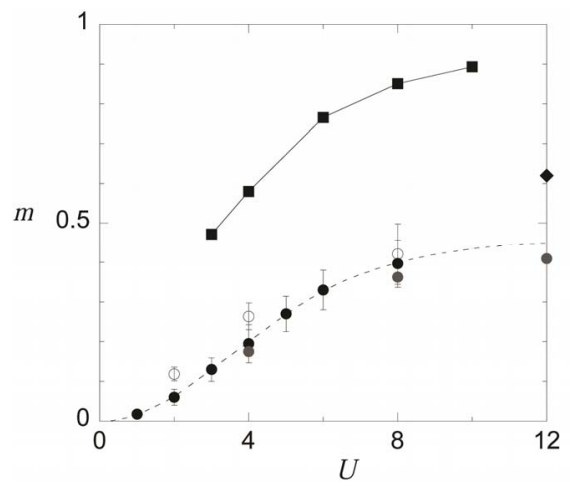

Figure 43. Magnetization as a function of $U$ for the halffilled Hubbard model after extrapolation at the limit of large $N$. Solid circles are the QMD results, and open circles are results obtained from the QMC method [28]. The squares are the Gutzwiller-VMC results [43] and gray solid circles show the 3rd $\lambda$-function $\left(\psi_{\lambda}^{(3)}\right)$ VMC results carried out on the $8 \times 8$ lattice [95]. The diamond symbol is the value from the two-dimensional Heisenberg model where $m=0.615 \quad[179,180]$.

$$
D_{\alpha \beta}(\ell)=\left\langle\Delta_{\alpha}^{\dagger}(i+\ell) \Delta_{\beta}(i)\right\rangle,
$$

where $\Delta_{\alpha}(i), \alpha=x, y$, denote the annihilation operators of the singlet electron pairs for the nearestneighbor sites:

$$
\Delta_{\alpha}(i)=c_{i \downarrow} c_{i+\hat{\alpha} \uparrow}-c_{i \uparrow} c_{i+\hat{\alpha} \downarrow} .
$$

Here $\hat{\alpha}$ is a unit vector in the $\alpha(=x, y)$-direction. We consider the correlation function of $d$-wave pairing:

$$
P_{d}(\ell)=\left\langle\Delta_{d}(i+\ell)^{\dagger} \Delta_{d}(i)\right\rangle
$$

where

$$
\Delta_{d}(i)=\Delta_{x}(i)+\Delta_{-x}(i)-\Delta_{y}(i)-\Delta_{-y}(i) .
$$

$i$ and $i+\ell$ denote sites on the lattice.

We show how the pair correlation function is evaluated in quantum Monte Carlo methods. We show the pair correlation functions $D_{y y}$ and $D_{y x}$ on the lattice $4 \times 3$ in Figures 44 and 45 . The boundary condition is open in the 4-site direction and is periodic in the other direction. An extrapolation is performed as a function of $1 / M$ in the QMC method with Metropolis algorithm and as a function of the energy variance $v$ in the QMD method with diagonalization. We keep $\Delta \tau$ a small constant $\simeq 0.02 \sim 0.05$ and and increase $\tau=\Delta \tau \cdot M$, where $M$ is the division number. In the Metropolis QMC method, we calculated averages over $5 \times 10^{5}$ Monte Carlo steps. The exact values were obtained by using the exact diagonalization method. Two methods give consistent results as shown in figures. All

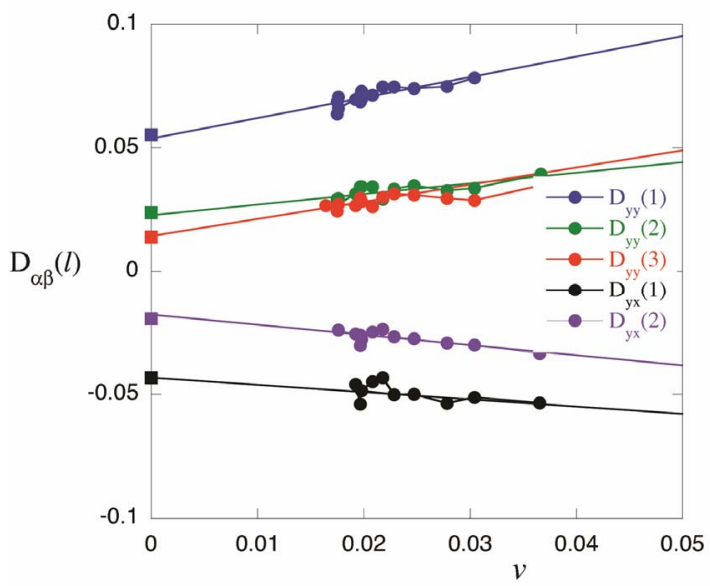

Figure 44. Pair correlation function $D_{y y}(\ell)$ and $D_{y x}(\ell)$ for $4 \times 3, U=4$ and $N_{e}=10$ obtained by the diagonalization quantum Monte Carlo method. The square are the exact results obtained by the exact diagonalization method. The data fit using a straight line using the least-square method as the variance is reduced. We started with $N_{\text {states }}=100$ (first solid circles) and then increase up to 2000. 


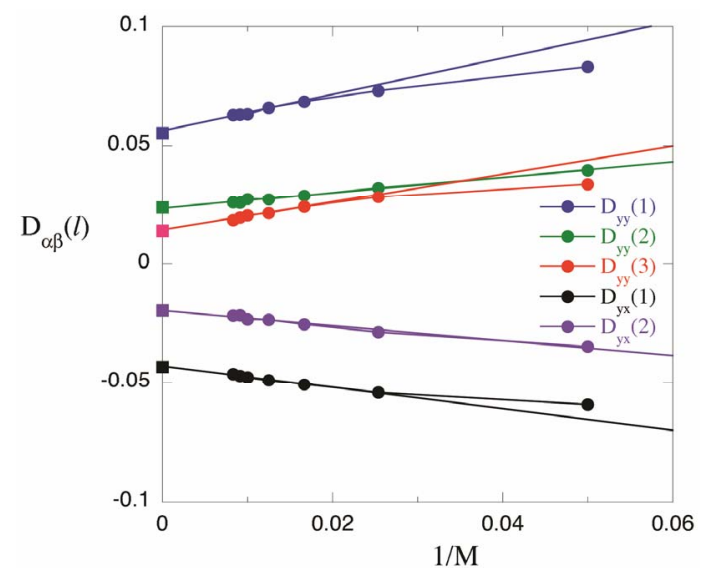

Figure 45. Pair correlation function $D_{y y}(\ell)$ and $D_{y x}(\ell)$ for $4 \times 3, U=4$ and $N_{e}=10$ obtained by the Metropolis quantum Monte Carlo method. The square are the exact results obtained by the exact diagonalization method. An extrapolation is performed as a function of $1 / M$.

the $D_{y y}(\ell)$ and $D_{y x}(\ell)$ are suppressed on $4 \times 3$ as $U$ is increased. In general, the pair correlation functions are suppressed in small systems. In Figures 46 and 47, we show the inter-chain pair correlation function for the ladder model $30 \times 2$. We use the open boundary condition. The number of electrons is $N_{e}=48$, and the strength of the Coulomb interaction is $U=4 . \Delta_{y}(i)$ indicates the electron pair along the rung, and $D_{y y}(\ell)$ is the expectation value of the parallel movement of the pair along the ladder. The results obtained by two methods are in good agreement except $\ell=(1,0)$ (nearest-neighbor correlation).

We first consider the half-filled case with $t^{\prime}=0$; in this case the antiferromagnetic correlation is dominant over the superconductive pairing correlation and thus the pairing correlation function is suppressed as the Coulomb repulsion $U$ is increased. The Figure 48 exhibits the $d$-wave pairing correlation function $P_{d}$ on $8 \times 8$ lattice as a function of the distance. The $P_{d}$ is suppressed due to the on-site Coulomb interaction, as expected. Its reduction is, however, not so considerably large compared to previous QMC studies [39] where the pairing correlation is almost annihilated for $U=4$. We then turn to the case of less than half-filling. We show the results on $8 \times 8$ with electron number $N_{e}=54$. We show $P_{d}$ as a function of the distance in Figure $49\left(N_{e}=54\right)$. In the scale of this figure, $P_{d}$ for $U>0$ is almost the same as that of the non-interacting case, and is enhanced slightly for large $U$. Our results indicate that the pairing correlation is not suppressed and is indeed enhanced by the Coulomb interaction $U$, and its enhancement is very small.

The Figure 50 shows $P_{d}$ on $10 \times 10$ lattice. This also indicates that the pairing correlation function is

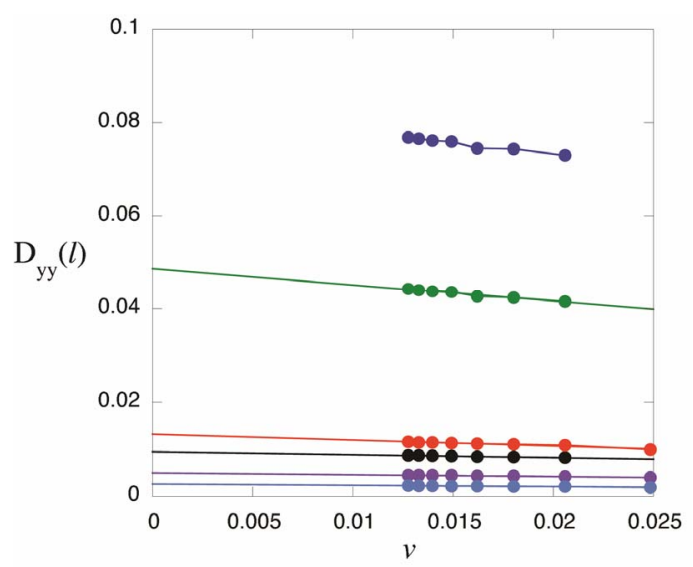

Figure 46. Pair correlation function $D_{y y}(\ell)$ as a function of the energy variance $v$ for $30 \times 2, U=4$ and $N_{e}=48$ obtained by the diagonalization quantum Monte Carlo method.

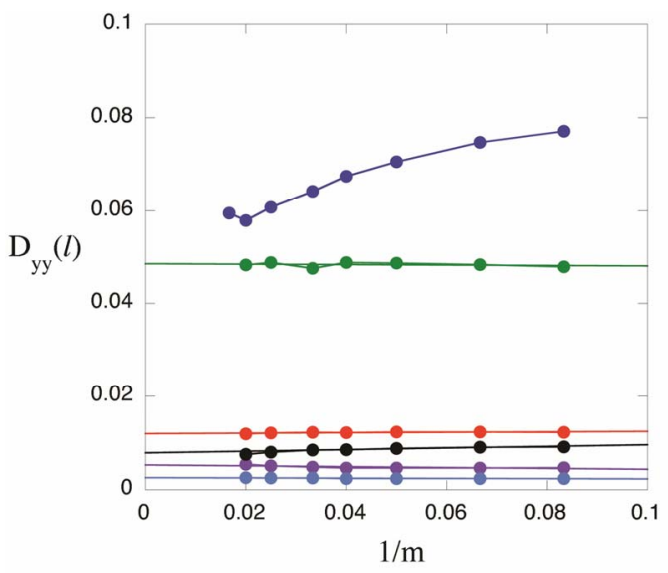

Figure 47. Pair correlation function $D_{y y}(\ell)$ as a function of $1 / M$ for $30 \times 2, U=4$ and $N_{e}=48$ obtained by the Metropolis quantum Monte Carlo method.

enhanced for $U>0$. There is a tendency that $P_{d}$ is easily suppressed as the system size becomes small. We estimated the enhancement ratio compared to the non-interacting case $\left.P_{d}(\ell)\right|_{U} /\left.P_{d}(\ell)\right|_{U=0}$ at $|\ell| \sim L / 2$ for $n_{e} \sim 0.8$ as shown in Figure 51. This ratio increases as the system size is increased. To compute the enhancement, we picked the sites, for example on $8 \times 8$ lattice, $\ell=(3,2),(4,0),(4,1),(3,3),(4,2),(4,3),(5,0)$, $(5,1)$ with $|\ell| \sim 4-5$ and evaluate the mean value. In our computations, the ratio increases almost linearly indicating a possibility of superconductivity. This indicates $P_{d}(\ell) \sim L P_{d}(\ell) \sim \ell P_{d}(\ell)$ for $\ell \sim L$.

Because $\left.P_{d}(\ell)\right|_{U=0} \sim 1 /|\ell|^{3}$, we obtain $P_{d}(\ell) \sim \ell P_{d}(\ell) \sim 1 /|\ell|^{2}$ for $|\ell| \sim L$. This indicates that the exponent of the power law is 2 . When $U=2$, the 


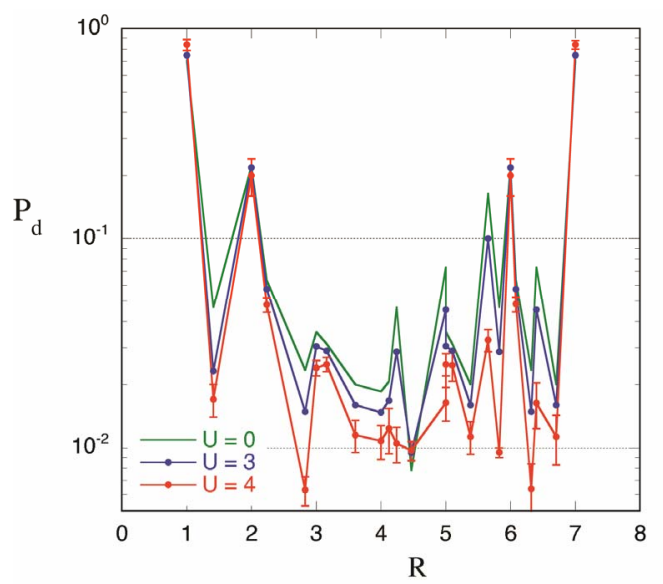

Figure 48. Pair correlation function $P_{d}$ as a function of the distance $R=|\ell|$ on $8 \times 8$ lattice for the half-filled case $N_{e}=64$. We set $t^{\prime}=0.0$ and $U=0,3$ and 4 . To lift the degeneracy of electron configurations at the Fermi energy in the half-filled case, we included a small staggered magnetization $\sim 1^{-4}$ in the initial wave function $\psi_{0}$.

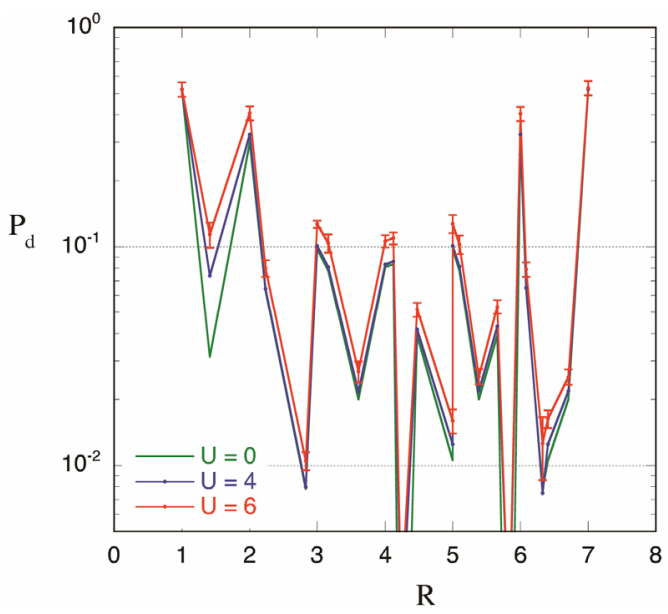

Figure 49. Pair correlation function $P_{d}$ as a function of the distance $R=|\ell|$ on $8 \times 8$ lattice for $N_{e}=54$. We set $t^{\prime}=-0.2$ and $U=0,4$ and 6 .

enhancement is small and is almost independent of $L$. In the low density case, the enhancement is also suppressed being equal to 1 . In Figure 52, the enhancement ratio is shown as a function of the electron density $n_{e}$ for $U=4$. A dome structure emerges even in small systems. The square in Figure 52 indicates the result for the half-filled case with $t^{\prime}=-0.2$ on $8 \times 8$ lattice. This is the open shell case and causes a difficulty in computations as a result of the degeneracy due to partially occupied electrons. The inclusion of $t^{\prime}<0$ enhances $P_{d}$ compared to the case with $t^{\prime}=0$ on $8 \times 8$ lattice. $P_{d}$ is, however, not enhanced over the non-interacting case at half-filling. This also holds for

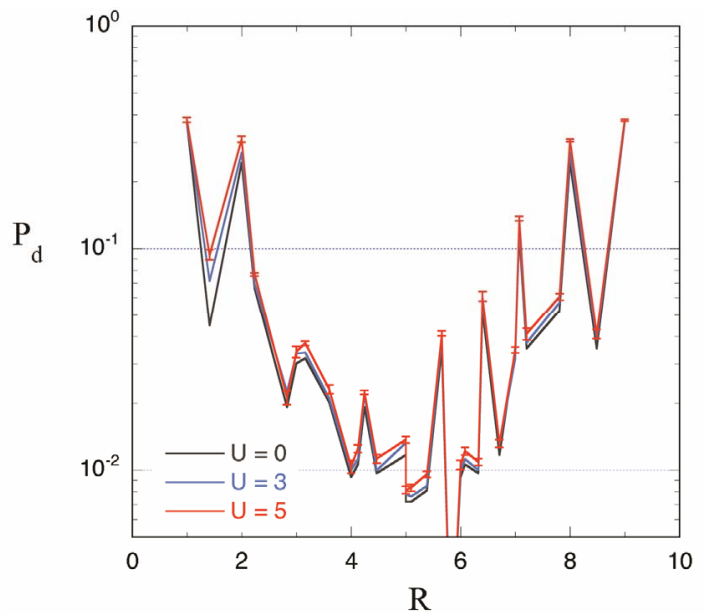

Figure 50. Pair correlation function $P_{d}$ as a function of the distance $R=|\ell|$ on $10 \times 10$ lattice for $N_{e}=82$ and $t^{\prime}=-0.2$. The strength of the Coulomb interaction is $U=\mathbf{0}, 3$ and 5 .

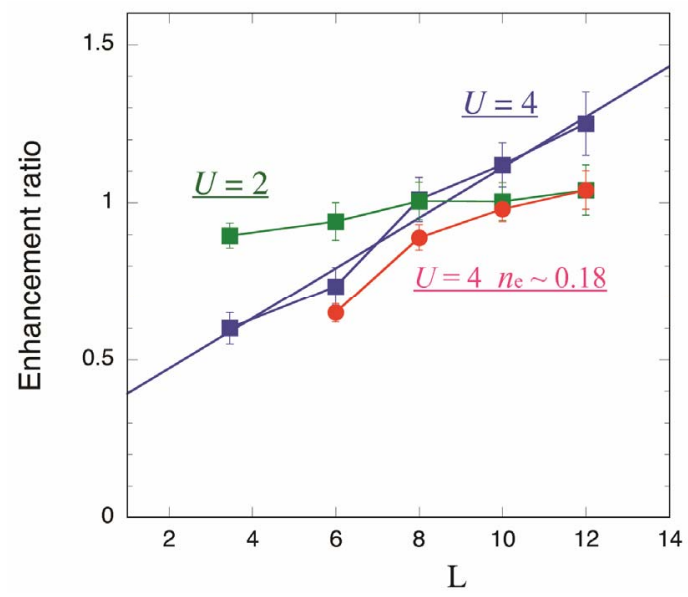

Figure 51. Enhancement ratio of pair correlation function $\left.\boldsymbol{P}_{d}\right|_{U} /\left.\boldsymbol{P}_{d}\right|_{U=0}$ as a function of the linear system size $L$ for $U=4$ and $U=2$. The electron density $n_{e}$ is about 0.8: $n_{e} \sim 0.8$ for squares. The data for $U=4$ and $n_{e} \sim 0.18$ are also shown by circles.

$10 \times 10$ lattice where the enhancement ratio $\sim 1$. This indicates the absence of superconductivity at half-filling.

\subsection{Spin Susceptibility}

We propose a method to compute the magnetic susceptibility at absolute zero $(T=0)$ [178]. We add the source term $H_{1}$ to the Hamiltonian as follows

$$
H_{1}=g \sum_{j} c_{j \uparrow}^{\dagger} c_{j \downarrow} \mathrm{e}^{i q \cdot R_{j}}+\text { h.c. }=g\left(S_{-q}^{+}+\text {h.c. }\right) \text {, }
$$

where $g$ is a small real number of the order $10^{-3}$ or $10^{-4}$. We calculate $-\left\langle S_{q}^{-}\right\rangle / g$ in the ground state, which 
is, as shown by the linear response theory, the magnetic susceptibility

$$
-\left\langle S_{\boldsymbol{q}}^{-}\right\rangle / g=\left.\int_{-\infty}^{\infty} \mathrm{d} t G_{r e t}(t, \boldsymbol{q})\right|_{T \rightarrow 0}=\left.\chi^{-+}(\boldsymbol{q}, \omega=0)\right|_{T \rightarrow 0},(10
$$

in the limit of small $g$, where $G_{r e t}$ is the retarded Green function and $\chi^{-+}(\boldsymbol{q}, \omega)$ is the dynamical susceptibility,

$$
\chi^{-+}(\boldsymbol{q}, \omega)=i \int_{0}^{\infty} \mathrm{d} t \mathrm{e}^{i \omega t}\left\langle\left[S_{\boldsymbol{q}}^{-}(t), S_{-\boldsymbol{q}}^{+}(0)\right]\right\rangle .
$$

Indeed, the above formula gives the correct spin susceptibility $\chi^{-+}(\mathbf{q}, \omega=0)$ on the finite lattice for the noninteracting case, which is given by $\sum_{k}\left(f\left(\xi_{k+q}\right)-f\left(\xi_{k}\right)\right) /\left(\xi_{k}-\xi_{k+q}\right)$ with the Fermi distribution function $f(\xi)$. We calculate $-\left\langle S_{q}^{-}\right\rangle / g$ by using the quantum Monte Carlo method to obtain $\chi^{-+}(\boldsymbol{q}, \omega=0)$.

We examine the results obtained for the susceptibilities. Figure $\mathbf{5 3}$ shows the spin susceptibility $\chi(Q)=\chi^{-+}(Q, \omega=0)$ for $Q=(\pi, \pi)$ on a $6 \times 2$ lattice as a function of $1 / m$ or the energy variance $v$. The number of electrons is 10 . The expectation values agree well with exact values given by the exact diagonalization method.

We now compute the staggered susceptibility $\chi_{\text {stag }}^{-+}$ by adding the source term

$H_{1}=g \sum_{j}\left(c_{j \uparrow}^{\dagger} c_{j \downarrow}(-1)^{j_{x}+j_{y}}+\right.$ h.c. $)$ to the Hamiltonian, where $j=\left(j_{x}, j_{y}\right)$. Here we set the periodic and antiperiodic boundary conditions in the $x$ and $y$ directions, respectively, to avoid a numerical difficulty caused by the degeneracy between states $\boldsymbol{k}$ and $\boldsymbol{k}+Q$

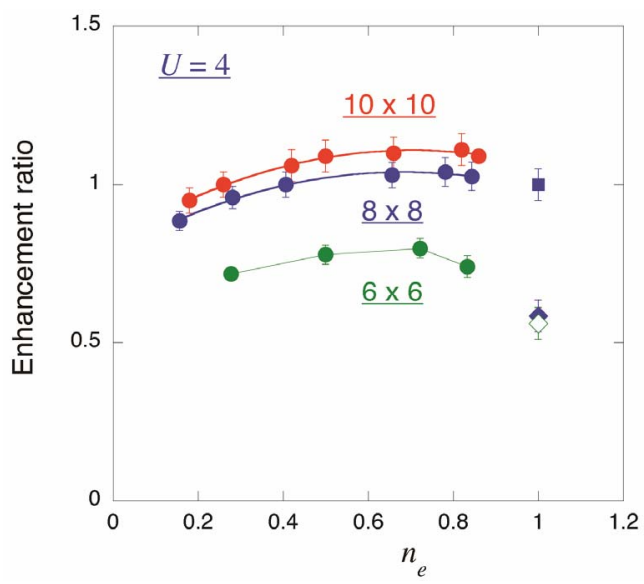

Figure 52. Enhancement ratio of pair correlation function $\left.\boldsymbol{P}_{d}\right|_{U} /\left.\boldsymbol{P}_{d}\right|_{U=0}$ as a function of the electron density $\boldsymbol{n}_{e}$. We adopt $t^{\prime}=-0.2$ and $U=4$. For the half-filled case, the diamonds show that for $t^{\prime}=0$ on $8 \times 8$ lattice (solid diamond) and $6 \times 6$ lattice (open diamond). The square is for $t^{\prime}=-0.2$ on $8 \times 8$ and $10 \times 10$ where there is no enhancement.

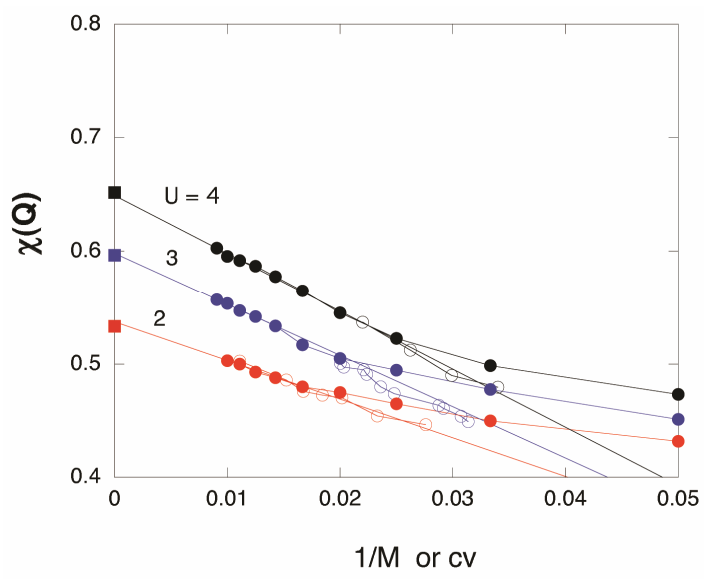

Figure 53. Spin susceptibility $\chi(Q)$ as a function of $1 / M$ or the variance $v$ for a $6 \times 2$ lattice with the periodic boundary condition. The number of electrons is 10 . We set $\Delta \tau=0.01$. The solid circles and open circles are obtained by using the QMC method and the QMD method, respectively. The squares indicate exact values. The variance $v$ is multiplied by a numerical constant. We set $\boldsymbol{U}=\mathbf{2}, \mathbf{3}$, and 4 in units of $t$.

where $Q=(\pi, \pi)$. It has been shown that a long-range spin correlation exists in the ground state of the half-filled Hubbard model with $t^{\prime}=0$ for $U>0$ [40, $41,179,180]$. In the case $U=0, \chi_{\text {stag }}^{-+}$exhibits a double logarithmic behavior $(\log (L))^{2} \cdot \chi_{\text {stag }}^{-+}$is shown as a function of $L$ in Figure 54 for $U=2,3$, and 4. The obtained values are well fitted by $L^{4}$ and $\chi_{\text {stag }}^{-+}$ diverges in the limit of a large system size $L$ :

$$
\chi_{\text {stag }}^{-+} \sim L^{4}
$$

This result is consistent with the existence of the long-range spin correlation for $U>0 \quad[179,180]$. The degree of divergence of $\chi_{\text {stag }}^{-+}$is beyond the criterion of the Kosterlitz-Thouless transition, and thus the long-range order represented by $\chi_{\text {stag }}^{-+}$belongs to a different category. The $L^{4}$ behavior of $\chi_{\text {stag }}$ is consistent with the predictions of perturbation theory in the 2D non-linear sigma model at low temperatures [181].

\subsection{Pair Susceptibility}

In this section we consider a method to evaluate the pair susceptibility $\chi_{\text {pair }}$ at $T=0$. In order to compute the pair susceptibility, we use an electron-hole transformation for the down spin, $c_{i \downarrow}=d_{i}^{\dagger}$, whereas the up-spin electrons are unaltered, $c_{i \uparrow}=c_{i}$. For the on-site $s$-wave pairing, the source term is given by the following expression

$$
H_{1}^{s}=g \sum_{i}\left(c_{i}^{\dagger} d_{i}+\text { h.c. }\right)
$$




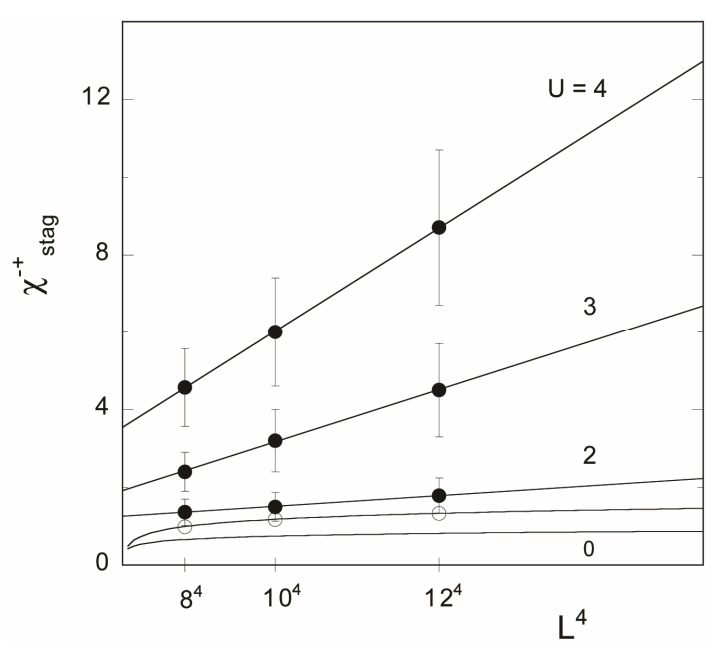

Figure 54. Staggered spin susceptibility $\chi_{\text {stag }}^{-+}$as a function of $L^{4}$ at half-filling with $t^{\prime}=0$ for $U=2,3$, and 4. We use the periodic and antiperiodic boundary conditions in the $x$ and $y$ directions, respectively. The lowest line is for $U=0$, which is fitted by a logarithmic curve. The open circles show the results for the Gutzwiller function with $U=4$, which exhibits a logarithmic dependence.

For the anisotropic $d$-wave pairing, we add

$$
\begin{aligned}
& H_{1}^{d}=g \sum_{i, \mu(= \pm x, \pm y)}\left(a_{\mu} c_{i+\mu}^{\dagger} c_{i \downarrow}^{\dagger}+\text { h.c. }\right) \\
& =g \sum_{i, \mu(= \pm x, \pm y)}\left(a_{\mu} c_{i+\mu}^{\dagger} d_{i}+\text { h.c. }\right)
\end{aligned}
$$

where $a_{ \pm x}=1$ and $a_{ \pm y}=-1$. The $s$-wave and $d$-wave pair susceptibility are respectively:

$$
\begin{array}{r}
\chi_{\text {pair }}^{s}=-\frac{1}{g} \frac{1}{N} \sum_{i}\left(\left\langle c_{i}^{\dagger} d_{i}\right\rangle+\text { h.c. }\right), \\
\chi_{\text {pair }}^{d}=-\frac{1}{g} \frac{1}{N} \sum_{i, \mu(= \pm x, \pm y)}\left(a_{\mu}\left\langle c_{i+\mu}^{\dagger} d_{i}\right\rangle+\text { h.c. }\right) .
\end{array}
$$

Using the Fourier transformation, the source term for the pair potential is written as follows

$H_{1}^{a}=g \sum_{k} z_{k}\left(c_{-k \downarrow}^{\dagger} c_{k \uparrow}^{\dagger}+\right.$ h.c. $)$ for $a=s$ or $d$ with the $k$-dependence factor $z_{k}$. If we define $\Delta_{k}=\left\langle c_{k \uparrow} c_{-k \downarrow}\right\rangle$, then for a small value of $g$, we have the following

$$
\Delta_{k} / g=-\sum_{k^{\prime}} z_{k^{\prime}} \int_{-\infty}^{\infty} \mathrm{d} t^{\prime} G_{r e t}\left(t-t^{\prime} ; k, k^{\prime}\right)
$$

where

$$
G_{r e t}\left(t-t^{\prime} ; k, k^{\prime}\right)=i \theta\left(t-t^{\prime}\right)\left\langle\left[b_{k}(t), b_{k^{\prime}}^{\dagger}\left(t^{\prime}\right)\right]\right\rangle,
$$

for $b_{k}=c_{k \uparrow} c_{-k \downarrow}$ and $b_{k}(t)=\mathrm{e}^{i H t} b_{k} \mathrm{e}^{-i H t}$. On the basis of analytic continuation, using the thermal Green function, $\Delta_{k}$ is written as

$$
\Delta_{k} / g=-\sum_{k^{\prime}} \int_{0}^{\beta} \mathrm{d} \tau \mathrm{e}^{i \omega_{n} \tau}\left\langle T_{\tau} b_{k}(\tau) b_{k^{\prime}}^{\dagger}(0)\right\rangle_{i \omega_{n} \rightarrow 0} .
$$

In the noninteracting system, this formula exhibits logarithmic divergence on the finite lattice $L \times L$ : $\chi_{\text {pair }}=A\left\langle\left|z_{k}\right|^{2}\right\rangle \log (c L)$ with constants $A$ and $c$, which can be confirmed by numerical estimations on finite systems.

In the Kosterlitz-Thouless theory, the susceptibility is scaled as follows [182,183]: $\chi \sim \xi^{2-\eta}$, where $\xi$ is the coherence length. $\xi$ is of order $L$ on a lattice $L \times L$ if long-range coherence exists. The exponent $\eta$ is expected to be 0 at absolute zero. Thus $\chi$ scales as $\chi \sim L^{2}$ in the ground state if the Kosterlitz-Thouless transition occurs at some temperature.

First, we investigate $\chi_{\text {pair }}^{s}$ for the attractive Coulomb interaction $U<0$. For this model, the existence of a Kosterlitz-Thouless transition has been predicted on the basis of quantum Monte Carlo methods [34,183]. The results in Figure 55 show that the size dependence for $t^{\prime}=0$ and $n_{e} \sim 0.8$ is

$$
\chi_{\text {pair }}^{s} \sim L^{2},
$$

which is consistent with previous studies, and shows the existence of a Kosterlitz-Thouless transition for the attractive interaction. At near half-filling, $\chi_{\text {pair }}^{s}$ is more enhanced than that at $n_{e} \sim 0.8$. Second, let us investigate the $d$-wave pair susceptibility $\chi_{\text {pair }}^{d}$ for the repulsive Coulomb interaction. Pair susceptibilities are

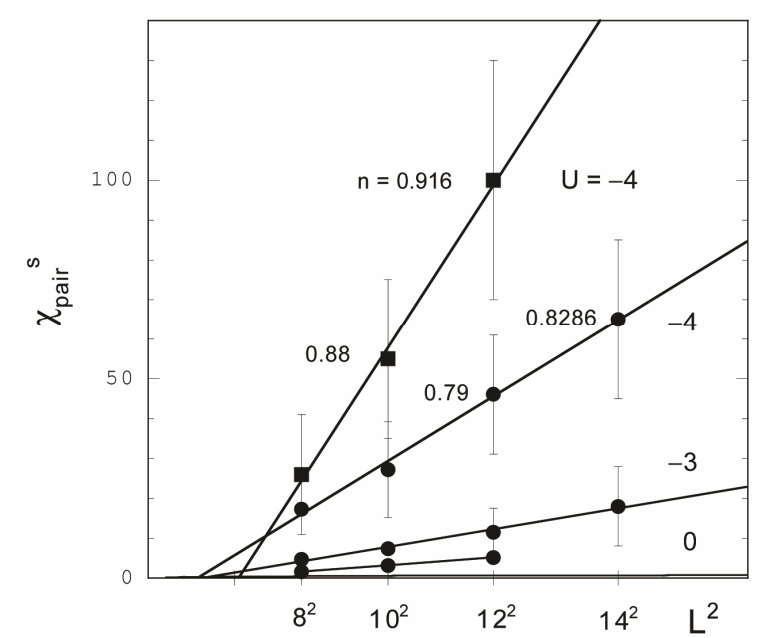

Figure 55. Isotropic $S$-wave susceptibility $\chi_{\text {pair }}^{s}$ as a function of $L^{2}$ for the negative- $U$ Hubbard model with $U=-2,-3$, and -4 , and $t^{\prime}=0$. The circles indicate the results for $n_{e} \sim 0.8$, where we use the periodic boundary condition in both the $x$ and $y$ directions, and the chemical potential is set at the center of the level spacing between adjacent energy levels. The lowest dotted line is for $U=0$ $\left(n_{e} \sim 0.75\right)$, which is fitted by a logarithmic curve, that is, $\chi_{\text {pair }}^{s} \sim \log (L)$. We show $\chi_{\text {pair }}^{s}$ for $n_{e} \sim 0.9$ and $U=-4$ by squares, where the boundary condition is antiperiodic in one direction and periodic in the other direction. 
sensitively dependent on the band structure, particularly the energy of the van Hove singularity, as a characteristic of two-dimensional systems. We compute $\chi_{\text {pair }}^{d}$ at an electron density $n_{e} \sim 0.87$, a value near that of optimally doped high-temperature cuprates. We set $t^{\prime}=-0.2$. Figure 56 shows $\chi_{\text {pair }}^{d}$ as a function of $L^{2}$ for $U=2,3,4$, and 5 with $t^{\prime}=-0.2$ and $n_{e} \sim 0.87$. This shows that

$$
\chi_{\text {pair }}^{d} \sim L^{2}
$$

if $U$ is moderately large. This result shows that a $d$-wave superconducting Kosterlitz-Thouless transition may exist for the repulsive interaction if we adjust the band parameters in the region of optimal doping.

\section{Summary}

We have investigated the superconductivity of electronic origin on the basis of the (single-band and three-band) two-dimensional Hubbard model. First, we employ the variational Monte Carlo method to clarify the phase diagram of the ground state of the Hubbard model. The superconducting condensation energy per site obtained by the Gutzwiller ansatz is reasonably close to experimental value $0.17 \sim 0.26 \mathrm{meV} /$ site . We have examined the stability of striped and checkerboard states in the under-doped region. The relation of the incommensurability and hole density, $\delta \sim x$, is satisfied in the

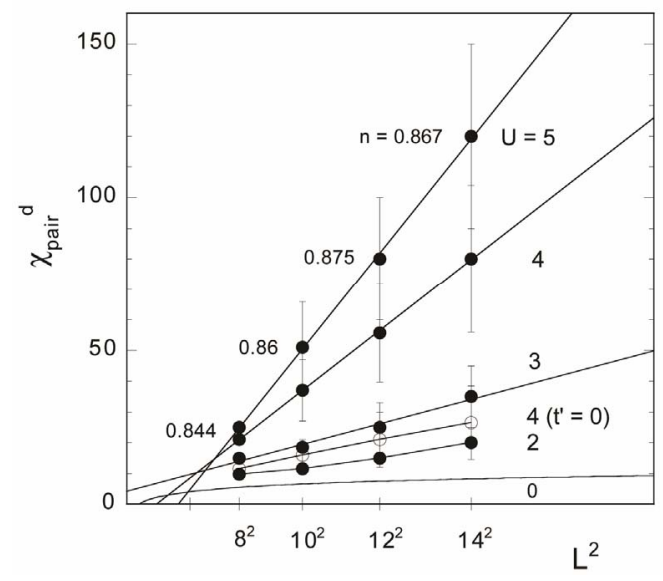

Figure 56. The $d$-wave susceptibility $\chi_{\text {pair }}^{s}$ as a function of $L^{2}$ for the repulsive- $U$ Hubbard model with $U=2,3,4$, and 5 . We use the periodic boundary condition in both the $x$ and $y$ directions. The solid circles present the results with $t^{\prime}=-0.2$ and $n_{e} \sim 0.87$ for $U=2,3,4$ and 5. For the solid squares the parameters are $t^{\prime}=-0.1$ and $n_{e} \sim 0.82$ with $U=4$. The open squares are for $t^{\prime}=-0.2, n_{e} \sim 1$ (near half-filling) and $U=4$. The open circles indicate the results for $t^{\prime}=0, n_{e} \sim 0.85$ and $U=4$. The lowest line for $U=0$ is fitted by a logarithmic curve: $\chi_{\text {pair }}^{d} \sim \log (L)$. lower doped region. We have found that the $4 \times 4$ period checkerboard spin modulation is stabilized in the two-dimensional Hubbard model with the Bi-2212 type band structure.

We have further performed investigation by using the quantum Monte Carlo method that is an exact unbiased method. We have presented an algorithm of the quantum Monte Carlo diagonalization to avoid the negative sign problem in quantum simulations of many-fermion systems. We have computed $d$-wave pair correlation functions. In the half-filled case $P_{d}$ is suppressed for the repulsive $U>0$, and when doped away from half-filling $N_{e}<N, P_{d}$ is enhanced slightly for $U>0$. It is noteworthy that the correlation function $P_{d}$ is indeed enhanced and is increased as the system size increases in the 2D Hubbard model. The enhancement ratio increases almost linearly $\propto L$ as the system size is increased, which is an indication of the existence of superconductivity. Our criterion is that when the enhancement ratio as a function of the system size $L$ is proportional to a certain power of $L$, superconductivity will be developed. This ratio depends on $U$ and is reduced as $U$ is decreased. The dependence on the band filling shows a dome structure as a function of the electron density. In the $10 \times 10$ system, the ratio is greater than 1 in the range $0.3<n_{e}<0.9$. Let us also mention on superconductivity at half-filling. Our results indicates the absence of superconductivity in the half-filling case because there is no enhancement of pair correlation functions

\section{Acknowledgements}

We thank I. Hase, S. Koikegami, S. Koike and J. Kondo for stimulating discussions. This work was supported by a Grant-in Aid for Scientific Research from the Ministry of Education, Culture, Sports, Science and Technology of Japan, and CREST Program of Japan Science and Technology Agency. A part of numerical calculations was performed at the facilities in the Supercomputer Center of the Institute for Solid State Physics of the University of Tokyo.

\section{REFERENCES}

[1] J. G. Bednorz and K. A. Müller, Zeitschrift für Physik B Condensed Matter, Vol. 64, 1986, pp. 189-193. doi:10.1007/BF01303701

[2] E. Dagotto, Reviews of Modern Physics, Vol. 66, 1994, pp. 763-840.

[3] P. W. Anderson, "The Theory of Superconductivity in the High-T ${ }^{\mathrm{c}}$ Cuprates," Princeton University Press, Princeton, 1997.

[4] T. Moriya and K. Ueda, Advances in Physics, Vol. 49, 2000, pp. 555-606. doi:10.1080/000187300412248

[5] K. H. Bennemann and J. B. Ketterson, "The Physics of 
Superconductor," Springer, Berlin, 2003.

[6] G. R. Stewart, Reviews of Modern Physics, Vol. 56, 1984, pp. 755-787.

[7] P. A. Lee, T. M. Rice, J. W. Serene, L. J. Sham and J. W. Wilkins, Comments on Condensed Matter Physics, Vol. 12, 1986, p. 99.

[8] H. R. Ott, Progress in Low Temperature Physics, Vol. 11, 1987, pp. 215-289. doi:10.1016/S0079-6417(08)60034-7

[9] M. B. Maple, "Handbook on the Physics and Chemistry of Rare Earths Vol. 30," Elsevier, Amsterdam, 2000.

[10] T. Ishiguro, K. Yamaji and G. Saito, "Organic Superconductors," Springer-Verlag, Berlin, 1998. doi:10.1007/978-3-642-58262-2

[11] C. C. Tsuei, et al., Physical Review Letters, Vol. 73, 1994, pp. 593-596.

[12] D. A. Wollman, et al., Physical Review Letters, Vol. 74, 1995, pp. 797-800.

[13] C. C. Tsuei and J. R. Kirtlry, Physical Review Letters, Vol. 85, 2000, pp. 182-185.

[14] T. Sato, T. Kamiyama, T. Takahashi, K. Kurahashi and K. Yamada, Science, Vol. 291, 2001, pp. 1517-1519. doi:10.1126/science.1058021

[15] T. Yanagisawa, S. Koikegami, H. Shibata, S. Kimura, S. Kashiwaya, A. Sawa, N. Matsubara and K. Takita, Journal of the Physical Society of Japan, Vol. 70, 2001, pp. 2833-2835. doi:10.1143/JPSJ.70.2833

[16] G. Shirane, Y. Endoh, R. Birgeneau, M. A. Kastner, Y. Hidaka, M. Oka, M. Suzuki and T. Murakami, Physical Review Letters, Vol. 59, 1987, p. 1613.

[17] K. B. Lyons, P. A. Fleury, L. F. Schncemmeyer and J. V. Waszczak, Physical Review Letters, Vol. 60, 1988, pp. 732-735.

[18] E. Manousakis and R. Salvadoe, Physical Review Letters, Vol. 62, 1989, pp. 1310-1313.

[19] P. Prelovsek, Physics Letters A, Vol. 126, 1988, pp. 287290. doi:10.1016/0375-9601(88)90764-5

[20] M. Inui and S. Doniach, Physical Review B, Vol. 38, 1988, pp. 6631-6635.

[21] T. Yanagisawa, Physical Review Letters, Vol. 68, 1992, pp. 1026-1029.

[22] T. Yanagisawa and Y. Shimoi, Physical Review B, Vol. 48, 1993, pp. 6104-6110.

[23] T. Yanagisawa and Y. Shimoi, International Journal of Modern Physics B, Vol. 10, 1996, pp. 3383-3450. doi:10.1142/S0217979296001835

[24] T. Yanagisawa, S. Koike and K. Yamaji, Physical Review $B$, Vol. 64, 2001, p. 184509.

[25] J. M. Tranquada, B. J. Sternlieb, J. D. Axe, Y. Nakamura and S. Uchida, Nature, Vol. 375, 1995, pp. 561-563. doi: $10.1038 / 375561 \mathrm{a} 0$

[26] J. Hubbard, Proceedings of the Royal Society A, Vol. 276, 1963, pp. 238-257.

[27] J. E. Hirsch, Physical Review Letters, Vol. 51, 1983, pp. 1900-1903.

[28] J. E. Hirsch, Physical Review B, Vol. 31, 1985, pp. 4403-
4419.

[29] S. Sorella, E. Tosatti, S. Baroni, R. Car and M. Parrinell, International Journal of Modern Physics B, Vol. 2, 1988 , p. 993. doi:10.1142/S0217979288000822

[30] S. R. White, D. J. Scalapino, R. L. Sugar, E. Y. Loh, J. E. Gubernatis and R. T. Scalettar, Physical Review B, Vol. 40, 1989, pp. 506-516.

[31] M. Imada and Y. Hatsugai, Journal of the Physical Society of Japan, Vol. 58, 1989, pp. 3752-3780. doi:10.1143/JPSJ.58.3752

[32] S. Sorella, S. Baroni, R. Car and M. Parrinello, Europhysics Letters, Vol. 8, 1989, pp. 663-668.

[33] E. Y. Loh, J. E. Gubernatis, R. T. Scalettar, S. R. White, D. J. Scalapino and R. L. Sugar, Physical Review B, Vol. 41, 1990, pp. 9301-9307.

[34] A. Moreo, D. J. Scalapino and E. Dagotto, Physical Review B, Vol. 56, 1991, pp. 11442-11444.

[35] N. Furukawa and M. Imada, Journal of the Physical Society of Japan, Vol. 61, 1992, pp. 3331-3354. doi:10.1143/JPSJ.61.3331

[36] A. Moreo, Physical Review B, Vol. 45, 1992, pp. 50595601.

[37] S. Fahy and D. R. Hamann, Physical Review B, Vol. 43, 1991, pp. 765-779.

[38] S. Zhang, J. Carlson and J. E. Gubernatis, Physical Review B, Vol. 55, 1997, pp. 7464-7477.

[39] S. Zhang, J. Carlson and J. E. Gubernatis, Physical Review Letters, Vol. 78, 1997, pp. 4486-4489.

[40] T. Kashima and M. Imada, Journal of the Physical Society of Japan, Vol. 70, 2001, pp. 2287-2299. doi:10.1143/JPSJ.70.2287

[41] T. Yanagisawa, Physical Review B, Vol. 75, 2007, p. 224503.

[42] T. Yanagisawa, New Journal of Physics, Vol. 15, 2013, Article ID: 033012.

[43] H. Yokoyama and H. Shiba, Journal of the Physical Society of Japan, Vol. 56, 1987, p. 1490.

[44] C. Gros, R. Joynt and T. M. Rice, Physical Review B, Vol. 36, 1987, pp. 381-393.

[45] T. Nakanishi, K. Yamaji and T. Yanagisawa, Journal of the Physical Society of Japan, Vol. 66, 1997, pp. 294-297. doi:10.1143/JPSJ.66.294

[46] K. Yamaji, T. Yanagisawa, T. Nakanishi and S. Koike, Physica C, Vol. 304, 1998, pp. 225-238.

[47] T. Yanagisawa, S. Koike and K. Yamaji, Journal of Physics: Condensed Matter, Vol. 14, 2002, pp. 21-31.

[48] T. Yanagisawa, S. Koike, S. Koikegami and K. Yamaji, Physical Review B, Vol. 67, 2003, p. 132400.

[49] T. Yanagisawa, M. Miyazaki and K. Yamaji, Journal of the Physical Society of Japan, Vol. 74, 2005, pp. 835-838. doi:10.1143/JPSJ.74.835

[50] M. Miyazaki, K. Yamaji and T. Yanagisawa, Journal of the Physical Society of Japan, Vol. 73, 2004, pp. 16431646. doi:10.1143/JPSJ.73.1643

[51] K. Yamaji and Y. Shimoi, Physica C, Vol. 222, 1994, pp. 
349-360. doi:10.1016/0921-4534(94)90553-3

[52] K. Yamaji, Y. Shimoi and T. Yanagisawa, Physica C, Vol. 235-240, 1994, pp. 2221-2222.

[53] S. Koike, K. Yamaji and T. Yanagisawa, Journal of the Physical Society of Japan, Vol. 68, 1999, pp. 1657-1663. doi:10.1143/JPSJ.68.1657

[54] S. Koike, K. Yamaji and T. Yanagisawa, Journal of the Physical Society of Japan, Vol. 69, 2000, pp. 2199-2208. doi:10.1143/JPSJ.69.2199

[55] R. M. Noack, S. R. White and D. J. Scalapino, Physica C, Vol. 270, 1996, pp. 281-296. doi:10.1016/S0921-4534(96)00515-1

[56] R. M. Noack, N. Bulut, D. J. Scalapino and M. G. Zacher, Physical Review B, Vol. 56, 1997, pp. 7162-7166.

[57] K. Kuroki, T. Kimura and H. Aoki, Physical Review B, Vol. 54, 1996, pp. R15641-R15644.

[58] S. Daul and D. J. Scalapino, Physical Review B, Vol. 62, 2000, pp. 8658-8660.

[59] K. Sano, Y. Ono and Y. Yamada, Journal of the Physical Society of Japan, Vol. 74, 2005, pp. 2885-2888. doi:10.1143/JPSJ.74.2885

[60] N. Bulut, Advances in Physics, Vol. 51, 2002, pp. 15871667. doi:10.1080/00018730210155142

[61] T. Aimi and M. Imada, Journal of the Physical Society of Japan, Vol. 76, 2007, p. 113708. doi:10.1143/JPSJ.76.113708

[62] J. Kondo, Journal of the Physical Society of Japan, Vol. 70, 2001, pp. 808-812. doi:10.1143/JPSJ.70.808

[63] R. Hlubina, Physical Review B, Vol. 59, 1999, pp. 96009605.

[64] S. Koikegami and T. Yanagisawa, Journal of the Physical Society of Japan, Vol. 70, 2001, pp. 3499-3502; Vol. 71, 2002, p. 761.

[65] S. Koikegami and T. Yanagisawa, Journal of the Physical Society of Japan, Vol. 75, 2006, Article ID: 034715. doi:10.1143/JPSJ.75.034715

[66] T. Yanagisawa, New Journal of Physics, Vol. 10, 2008, Article ID: 023014.

[67] A. B. Harris and R. V. Lange, Physical Review, Vol. 157, 1967, pp. 295-314. doi:10.1103/PhysRev.157.295

[68] M. C. Gutzwiller, Physical Review Letters, Vol. 10, 1963, pp. 159-162.

[69] J. Kanamori, Progress of Theoretical Physics, Vol. 30, 1963, pp. 275-289. doi:10.1143/PTP.30.275

[70] H. Bethe, Zeitschrift für Physik, Vol. 71, 1931, pp. 205226. doi:10.1007/BF01341708

[71] C. N. Yang, Physical Review Letters, Vol. 19, 1967, pp. 1312-1315.

[72] E. H. Lieb and F. Y. Wu, Physical Review Letters, Vol. 20, 1968, pp. 1445-1448.

[73] H. J. Schulz, Physical Review Letters, Vol. 64, 1990, pp. 2831-2834.

[74] H. Frahm and V. E. Korepin, Physical Review B, Vol. 42, 1990, pp. 10553-10565.

[75] N. Kawakami and S. K. Yang, Physics Letters A, Vol.
148,1990 , pp. $359-362$. doi:10.1016/0375-9601(90)90818-9

[76] F. D. M. Haldane, Journal of Physics C, Vol. 14, 1981, p. 2585.

[77] V. J. Emery, Physical Review Letters, Vol. 58, 1987, pp. 2794-2797.

[78] L. H. Tjeng, H. Eskes and G. A. Sawatzky, "Strong Correlation and Superconductivity," Springer, Berlin Heidelberg, 1989.

[79] W. H. Stephan, W. Linden and P. Horsch, Physical Review B, Vol. 39, 1989, pp. 2924-2927.

[80] J. E. Hirsch, E. Y. Loh, D. J. Scalapino and S. Tang, Physical Review B, Vol. 39, 1989, pp. 243-253.

[81] R. T. Scalettar, D. J. Scalapino, R. L. Sugar and S.R. White, Physical Review B, Vol. 44, 1991, pp. 770-781.

[82] G. Dopf, A. Muramatsu and W. Hanke, Physical Review $B$, Vol. 41, 1990, pp. 9264-9275.

[83] G. Dopf, A. Muramatsu and W. Hanke, Physical Review Letters, Vol. 68, 1992, pp. 353-356.

[84] T. Asahata, A. Oguri and S. Maekawa, Journal of the Physical Society of Japan, Vol. 65, 1996, pp. 365-368. doi:10.1143/JPSJ.65.365

[85] K. Kuroki and H. Aoki, Physical Review Letters, Vol. 76, 1996, pp. 4400-4403.

[86] T. Takimoto and T. Moriya, Journal of the Physical Society of Japan, Vol. 66, 1997, pp. 2459-2465. doi:10.1143/JPSJ.66.2459

[87] M. Guerrero, J. E. Gubernatis and S. Zhang, Physical Review B, Vol. 57, 1998, pp. 11980-11988.

[88] S. Koikegami and K. Yamada, Journal of the Physical Society of Japan, Vol. 69, 2000, pp. 768-776. doi:10.1143/JPSJ.69.768

[89] H. Eskes, G. A. Sawatzky and L. F. Feiner, Physica $C$, Vol. 160, 1989, pp. 424-430. doi:10.1016/0921-4534(89)90415-2

[90] M. S. Hybertsen, E. B. Stechel, M. Schlüter and D. R. Jennison, Physical Review B, Vol. 41, 1990, pp. 1106811072 .

[91] A. K. McMahan, J. F. Annett and R. M. Martin, Physical Review B, Vol. 42, 1990, pp. 6268-6282.

[92] D. Ceperley, G. V. Chester and K. H. Kalos, Physical Review B, Vol. 16, 1977, pp. 3081-3099.

[93] C. J. Umrigar, K. G. Wilson and J. W. Wilkins, Physical Review Letters, Vol. 60, 1988, pp. 1719-1722.

[94] R. Blankenbecler, D. J. Scalapino and R. L. Sugar, Physical Review D, Vol. 24, 1981, pp. 2278-2286.

[95] T. Yanagisawa, S. Koike and K. Yamaji, Journal of the Physical Society of Japan, Vol. 67, 1998, pp. 3867-3874. doi:10.1143/JPSJ.67.3867

[96] T. Yanagisawa, S. Koike and K. Yamaji, Journal of the Physical Society of Japan, Vol. 68, 1999, pp. 3608-3614.

[97] K. Yamaji, T. Yanagisawa and S. Koike, Physica B, Vol. 284-288, 2000, p. 415.

[98] L. F. Feiner, J. H. Jefferson and R. Raimondi, Physical Review B, Vol. 53, 1996, pp. 8751-8773. 
[99] J. W. Loram, K. A. Mirza, J. R. Cooper and W. Y. Liang, Physical Review Letters, Vol. 71, 1993, pp. 1740-1750.

[100] P. W. Anderson, Science, Vol. 279, 1998, pp. 1196-1198. doi:10.1126/science.279.5354.1196

[101] Z. Hao, J. R. Clem, M. W. McElfresh, L. Civale, A. P. Malozemoff and F. Holtzberg, Physical Review B, Vol. 43, 1991, pp. 2844-2852.

[102] H. Yokoyama and M. Ogata, Journal of the Physical Society of Japan, Vol. 65, 1996, pp. 3615-3629. doi:10.1143/JPSJ.65.3615

[103] T. Matsuzaki, N. Momono, M. Oda and M. Ido, Journal of the Physical Society of Japan, Vol. 73, 2004, pp. 22322238. doi:10.1143/JPSJ.73.2232

[104] T. Tohyama and S. Maekawa, Superconductor Science and Technology, Vol. 13, 2000, p. 17.

[105] M. Miyazaki, K. Yamaji and T. Yanagisawa, Journal of Physics and Chemistry of Solids, Vol. 63, 2002, pp. 14031407. doi:10.1016/S0022-3697(02)00072-0

[106] T. Yanagisawa, M. Miyazaki and K. Yamaji, Journal of the Physical Society of Japan, Vol. 78, 2009, Article ID: 013706. doi:10.1143/JPSJ.78.013706

[107] D. J. Singh and W. E. Pickett, Physica C, Vol. 203, 1992, pp. 193-202. doi:10.1016/0921-4534(92)90526-I

[108] N. E. Hussey, et al., Nature, Vol. 425, 2003, pp. 814-817.

[109] M. Plate, et al., Physical Review Letters, Vol. 95, 2005, Article ID: 07001.

[110] W. S. Lee, et al., 2006, arXiv: cond-mat/0600347.

[111] K. Yamaji, T. Yanagisawa, M. Miyazaki and R. Kadono, Physica C, Vol. 468, 2008, pp. 1125-1128. doi:10.1016/j.physc.2008.05.015

[112] K. Yamaji, T. Yanagisawa, M. Miyazaki and R. Kadono, Journal of the Physical Society of Japan, Vol. 80, 2011, Article ID: 083702. doi:10.1143/JPSJ.80.083702

[113] M. Fabrizio, A. Parola and T. Tosatti, Physical Review B, Vol. 46, 1992, pp. 3159-3162.

[114] M. Fabrizio, Physical Review B, Vol. 48, 1993, pp. 15838-15860.

[115] T. Yanagisawa, Y. Shimoi and K. Yamaji, Physical Review $B$, Vol. 52, 1995, pp. 3860-3863.

[116] L. Balents and M. P. A. Fisher, Physical Review B, Vol. 53, 1996, pp. 12133-12141.

[117] G.-Q. Zheng, Y. Kitaoka, K. Ishida and K. Asayama, Journal of the Physical Society of Japan, Vol. 64, 1995, pp. 2524-2532. doi:10.1143/JPSJ.64.2524

[118] J. Tranquada, J. D. Axe, N. Ichikawa, Y. Nakamura, S. Uchida and B. Nachumi, Physical Review B, Vol. 54, 1996, pp. 7489-7499.

[119] T. Suzuki, T. Goto, K. Chiba, T. Fukase, H. Kimura, K. Yamada, M. Ohashi and Y. Yamaguchi, Physical Review $B$, Vol. 57, 1998, pp. R3229-R3232.

[120] K. Yamada, C. H. Lee, K. Kurahashi, J. Wada, S. Wakimoto, S. Ueki, H. Kimura and Y. Endoh, Physical Review $B$, Vol. 57, 1998, pp. 6165-6172.

[121] M. Arai, T. Nishijima, Y. Endoh, T. Egami, S. Tajima, K. Tomimoto, Y. Shiohara, M. Takahashi, A. Garrett and S.
M. Bennington, Physical Review Letters, Vol. 83, 1999, pp. 608-611.

[122] H. A. Mook, D. Pengcheng, F. Dogan and R. D. Hunt, Nature, Vol. 404, 2000, pp. 729-731.

[123] S. Wakimoto, R. J. Birgeneau, M. A. Kastner, Y. S. Lee, R. Erwin, P. M. Gehring, S. H. Lee, M. Fujita, K. Yamada, Y. Endoh, K. Hirota and G. Shirane, Physical Review B, Vol. 61, 2000, pp. 3699-3706.

[124] T. Noda, H. Eisaki and S. Uchida, Science, Vol. 286, 1999, pp. 265-268. doi:10.1126/science.286.5438.265

[125] X.J. Zhou, P. Bogdanov, S. A. Keller, T. Noda, H. Eisaki, S. Uchida, Z. Hussain and Z. X. Shen, Science, Vol. 286, 1999, pp. 268-272.doi:10.1126/science.286.5438.268

[126] C. Niedermayer, C. Bernhard, T. Blasius, A. Golnik, A. Moodenbauch and J. I. Budnick, Physical Review Letters, Vol. 80, 1998, pp. 3843-3846.

[127] H. Kimura et al., Physical Review, Vol. 59, 1999, pp. 6517-6523.

[128] H. Matsushita, H. Kimura, M. Fujita, K. Yamada, K. Hirota and Y. Endoh, Journal of Physics and Chemistry of Solids, Vol. 60, 1999, pp. 1079-1081. doi:10.1016/S0022-3697(99)00052-9

[129] M. Matsuda, M. Fujita, K. Yamada, R. J. Birgeneau, M. A. Kastner, H. Hirai, Y. Endoh, S. Wakimoto and G. Shirane, Physical Review B, Vol. 62, 2000, pp. 91489154.

[130] M. Fujita, K. Yamada, H. Hiraka, P. M. Gehring, S. H. Leem S. Wakimoto and G. Shigane, Physical Review B, Vol. 65, 2002, Article ID: 064505.

[131] T. Giamarchi and C. Lhuillier, Physical Review B, Vol. 43, 1991, pp. 12943-12951.

[132] K. Machida, Physica C, Vol. 158, 1989, pp. 192-196. doi:10.1016/0921-4534(89)90316-X

[133] D. Poilblanc and T. M. Rice, Physical Review B, Vol. 39, 1989, pp. 9749-9752.

[134] M. Kato, K. Machida, H. Nakanishi and M. Fujita, Journal of the Physical Society of Japan, Vol. 59, 1990, pp. 1047-1058. doi:10.1143/JPSJ.59.1047

[135] H. Schulz, Physical Review Letters, Vol. 64, 1990, pp. 1445-1448.

[136] J. Zaanen and A. M. Oles, Annalen der Physik, Vol. 508, 1996, pp. 224-246.

[137] M. Ichioka and K. Machida, Journal of the Physical Society of Japan, Vol. 68, 1999, pp. 4020-4031. doi:10.1143/JPSJ.68.4020

[138] S. White and D. J. Scalapino, Physical Review Letters, Vol. 80, 1998, pp. 1272-1275.

[139] S. White and D. J. Scalapino, Physical Review Letters, Vol. 81, 1998, pp. 3227-3230.

[140] C. S. Hellberg and E. Manousakis, Physical Review Letters, Vol. 83, 1999, p. 132.

[141] K. Kobayashi and H. Yokoyama, Physica B, Vol. 259261, 1999, pp. 506-508.

[142] A. Himeda, T. Kato and M. Ogata, Physical Review Let- 
ters, Vol. 88, 2002, Article ID: 117001.

[143] W. Loram, K. A. Mirza, J. R. Cooper, N. Athanassopoulou and W. Liang, "Proceedings of the 10th Anniversary HTS Workshop,” World Scientific, Singapore, 1996.

[144] M. Miyazaki, K. Yamaji, T. Yanagisawa and K. Yonemitsu, Physcs Procedia, Vol. 27, 2012, pp. 64-67. doi:10.1016/j.phpro.2012.03.411

[145] H. Mukuda, et al., Physical Review Letters, Vol. 96, 2006, Article ID: 087001.

[146] J. E. Hoffman, et al., Science, Vol. 295, 2002, pp. 466469. doi:10.1126/science. 1066974

[147] W. D. Wise, et al., Nature Physics, Vol. 4, 2008, pp. 696-699.

[148] T. Hanaguri, et al., Nature, Vol. 430, 2004, pp. 10011005.

[149] S. R. White and D. J. Scalapino, Physical Review B, Vol. 70, 2004, p. 220506

[150] G. Seibold, J. Lorenzana and M. Grilli, Physical Review B, Vol. 75, 2007, p. 100505.

[151] M. Miyazaki, K. Yamaji, T. Yanagisawa and R. Kadono, Journal of the Physical Society of Japan, Vol. 78, 2009, Article ID: 043706. doi:10.1143/JPSJ.78.043706

[152] R. Jastrow, Physical Review, Vol. 55, 1955, pp. 14791484.

[153] H. Ohtsuka, Journal of the Physical Society of Japan, Vol. 61, 1992, pp. 1645-1656. doi:10.1143/JPSJ.61.1645

[154]H. de Raedt, Physics Reports, Vol. 127, 1985, pp. 233307. doi:10.1016/0370-1573(85)90044-4

[155] H. Yokoyama and H. Shiba, Journal of the Physical Society of Japan, Vol. 57, 1988, pp. 2482-2493. doi:10.1143/JPSJ.57.2482

[156] J. B. Torrance and R. M. Metzger, Physical Review Letters, Vol. 63, 1989, pp. 1515-1518.

[157] D. J. Scalapino, E. Loh and J. E. Hirsch, Physical Review $B$, Vol. 34, 1986, pp. 8190-8192.

[158] H. Shimahara and S. Takada, Journal of the Physical Society of Japan, Vol. 57, 1988, pp. 1044-1055. doi:10.1143/JPSJ.57.1044

[159] P. Monthoux, A. V. Balatsky and D. Pines, Physical Review Letters, Vol. 67, 1991, pp. 3448-3451.

[160] N. E. Bickers, D. J. Scalapino and S. R. White, Physical Review Letters, Vol. 62, 1989, pp. 961-964.

[161] C.-H. Pao and N. E. Bickers, Physical Review B, Vol. 49, 1994, pp. 1586-1599.

[162] P. Monthoux and D. J. Scalapino, Physical Review Letters, Vol. 72, 1994, pp. 1874-1877.

[163] Y. Yanase and K. Yamada, Journal of the Physical Society of Japan, Vol. 68, 1999, pp. 2999-3015.
doi:10.1143/JPSJ.68.2999

[164] K. Miyake, S. Schmidt-Rink and C. M. Varma, Physical Review B, Vol. 34, 1986, pp. 6554-6556.

[165] T. Moriya, Y. Takahashi and K. Ueda, Journal of the Physical Society of Japan, Vol. 59, 1990, pp. 2905-2915. doi:10.1143/JPSJ.59.2905

[166] T. Hotta, Journal of the Physical Society of Japan, Vol. 62, 1993, pp. 4414-4425. doi:10.1143/JPSJ.62.4414

[167] T. Jujo, S. Koikegami and K. Yamada, Journal of the Physical Society of Japan, Vol. 68, 1999, pp. 1331-1339. doi:10.1143/JPSJ.68.1331

[168] T. Nomura and K. Yamada, Journal of the Physical Society of Japan, Vol. 69, 2000, pp. 3678-3688. doi:10.1143/JPSJ.69.3678

[169] S. Koikegami, Y. Yoshida and T. Yanagisawa, Physical Review B, Vol. 67, 2003, p. 134517.

[170] S. Koikegami and T. Yanagisawa, Journal of the Physical Society of Japan, Vol. 79, 2010, Article ID: 064701. doi:10.1143/JPSJ.79.064701

[171] K. Yamaji and T. Yanagisawa, Physica C, 2013.

[172] S. Koike, et al., Physica C, Vol. 388-389, 2003, pp. 6567.

[173] E. Pavarini, et al., Physical Review Letters, Vol. 87, 2001, Article ID: 047003.

[174] T. Mizusaki, M. Honma and T. Otsuka, Physical Review C, Vol. 53, 1986, p. 2786.

[175] S. Sorella, Physical Review B, Vol. 64, 2001, Article ID: 024512.

[176] D. E. Goldberg, "Genetic Algorithms in Search, Optimization and Machine Learning," Addison-Wesley, Boston, 1989.

[177] A. Parola, S. Sorella, S. Baroni, R. Car, M. Parrinello and E. Tosatti, Physica C, Vol. 162-164, 1989, pp. 771-772.

[178] T. Yanagisawa, Journal of the Physical Society of Japan, Vol. 79, 2010, Article ID: 063708. doi:10.1143/JPSJ.79.063708

[179] J. A. Riera and A. P. Young, Physical Review B, Vol. 39, 1989, pp. 9697-9700.

[180] M. C. Buonaura and S. Sorella, Physical Review B, Vol. 57, 1998, pp. 11446-11456.

[181] P. Hasenfratz and F. Niedernayer, Zeitschrift für Physik B Condensed Matter, Vol. 92, 1993, pp. 91-112. doi:10.1007/BF01309171

[182] J. M. Kosterlitz and D. J. Thouless, Journal of Physics $C$, Vol. 6, 1973, pp. 1181-1203.

[183] S. Chandrasekharan and J. C. Osborn, Physical Review B, Vol. 66, 2002, Article ID: 045113. 